\title{
Green-gradient based canopy segmentation: A multipurpose image mining technique with potential use in crop phenotyping and canopy studies
}

\author{
Abbas Haghshenas ${ }^{1}$, Yahya Emam ${ }^{2 *}$ \\ ${ }^{1} \mathrm{PhD}$ student of Agronomy, Department of Crop Production and Plant Breeding, Shiraz \\ University, Shiraz, Iran. \\ ${ }^{2}$ Professor, Department of Crop Production and Plant Breeding, Shiraz University, Shiraz, \\ Iran. \\ ${ }^{*}$ Corresponding author. Email: yaemam@shirazu.ac.ir
}

\begin{abstract}
Vegetation canopies may be characterized based on how they interact with light. Efficient quantification of the sophisticated shading patterns inside the 3D vegetation canopies may improve our understanding of canopy functions and status, which is possible now more than ever, thanks to the high-throughput phenotyping (HTP) platforms. In order to evaluate the option of developing an image mining technique based on relative variations in RGB triplets (green-gradient based canopy segmentation method, or GSM) for HTP purposes and canopy assessments, an archive of ground-based nadir images of heterogeneous wheat canopies (cultivar mixtures) were analyzed. The images were taken from experimental plots of a twoyear field experiment conducted during 2014-15 and 2015-16 growing seasons at the School of Agriculture, Shiraz University, Iran. In GSM, the vegetation pixels were categorized into the maximum possible number of 255 groups based on their green levels. Subsequently, mean red and mean blue levels of each group were calculated and plotted against the green levels. It was observed that the two sets of red and blue mean values yielded two smooth upward exponential shaped curves, with the blue one having a higher degree of curvature. It is suggested that such graph (i.e. GSM graph) could be readily utilized for identifying and characterizing canopies, e.g. in the form of one or two image-derived equation(s). Moreover,
\end{abstract}


based on the relative variations in the local slopes of red or blue curves compared with the green linear trend, a curve segmentation approach i.e. $\mathrm{ST}_{3}$ was evaluated, by which each curve was separately divided into 5 distinct segments. Reconciling and comparing these curve-segments with the images, a precise classification of canopy pixels was resulted, which was in accordance with sun exposure. Besides, in order to assess the option of using the quantitative characteristics of GSM curves, 169 curve-derived attributes were defined and their potential relationships with grain yield $(\mathrm{GY})$ and number of grains $\mathrm{m}^{-2}(\mathrm{GN})$ were evaluated using various data mining models and approaches. The results indicated that the qualitative classes of GY and GN were predicted with high accuracies (even up to 100\%). In another assessment, it was found that the efficiency of predictions might be improved through a considerable (almost 80\%) reduction in the number of GSM-curve attributes (used as model inputs). Moreover, evidences for sensibility of GSM curves to phenology and environmental stresses are presented. It seems that the new approach may provide a multipurpose HTP platform and open new windows to canopy studies.

\section{Keywords}

Canopy structure, cultivar mixture, data mining, grain yield, shading pattern, yield components

\section{Introduction}

Vegetation canopies may be characterized based on how they interact with light. In plant communities, a theoretical flat (2D) arrangement of a single unshaded layer of (green) leaves would provide potentially the basic light conditions for photosynthesis; though, such conceptually integrated light-exposed surface is actually distributed in a sophisticated manner over a three dimensional structure, which inevitably casts a shadow on itself. Moreover, the shading pattern of canopies shows large tempo-spatial variations (e.g. due to time, season, plant growth stage, altering growth behaviors, biotic and abiotic stresses, etc.) which makes it even more challenging to approach coincidentally a comprehensive, efficient, and easily applicable characterizing framework. 
Attempts to respond the intensified needs for a modern way of observation in agricultural and plant sciences, has led to the era of high-throughput phenotyping (HTP), i.e. the ubiquitous utilization of the remotely quantifying platforms for a deeper sensation of vegetation canopies. With respect to the key roles predicted for HTP, e.g. in mapping the genotype to phenotype (Chen et al., 2014), its integration with genomics is even described as the potentially second green revolution (Tanger et al., 2017; Crain et al., 2018). Such a promising perspective is basically originated from the significant capacities of HTP for data collecting and processing (e.g. see Cabrera-Bosquet et al., 2012; Araus and Cairns, 2014; Zhang et al., 2017). Besides the advantages of HTP in producing huge amounts of data, the platforms efficiency is rarely considered (see Araus et al., 2018); which may be defined using the concept of the balance between the complexity of the sensing and/or computation units on one hand, and the resulted achievements on the other hand. For instance, the method efficiency is expected to be higher where adequate and reliable information about the crop canopy structure is easily achieved using image processing of a common digital image, compared with the case of using multiple types of sensors and/or sophisticated computation models, with a more or less similar outputs.

The high spectral overlap between electromagnetic radiation of visible light and photosynthetically active radiation (PAR, i.e. about 400-700 $\mathrm{nm}$ wavelengths, McCree, 1972), provides the opportunity of using digital cameras in crop phenotyping as both reliable and readily available sensors of remote sensing. Interestingly, most of the current low-cost approaches for crop phenotyping are based on exploitation of the possibilities opened by RGB images (Araus et al., 2018). Employing commercial digital cameras along with most often basic image processing techniques, an increasing number of studies have reported robust correlations between various image-derived indices and crop criteria including nitrogen status (Li et al., 2010; Wang et al., 2013; Lee and Lee, 2013), crop growth (Sakamoto et al., 2012; Lee and Lee, 2013), chlorophyll content (Hunt Jr et al., 2013), leaf area (Easlon and Bloom, 2014), leaf angle (Zou et al., 2014), selection criteria for breeding programs (Casadesús et al., 2007), etc.

As in many other fields of empirical sciences, sampling (for statistical analyses) is a fundamental factor in crop/plant physiological assessments; where probably the most significant power of digital cameras lies. A typical 8-megapixel color image taken from a low height above the crop canopy, for instance, may provide a huge physiological sample included (e.g. given an $80 \%$ canopy coverage) more than 6 million pixels analogous to 
individual real green points (probably of various sizes). In addition to spatial resolution, high color sensibilities (e.g. the ability of discriminating over 16 million - or $256^{3}$ - colors per pixel in a common 24-bit color depth) have made it possible to record an acceptable number of 256 different mono-color intensities of light reflected in either red, green, and blue bands for a single pixel (the range of 0 to 255 , for zero to maximum light sensed in a single band). Processing the certainly adequate data-set provided by such a unique light sampling tool, requires subsequently more efficient data management, computational approaches, and powerful interpretation of results (Araus and Cairns, 2014) in order to improve our understanding of crop phenotype. In other words, it seems that comparatively, a minor part of the computation capacity provided by RGB images has been explored.

Image mining of an archive of ground-based nadir images taken from the experimental plots of a field study consisted of monocultures and mixtures of early- to middle-ripening wheat cultivars, an accidental observation was made using the statistics of the RGB triplets, based on which the present study was designed. We were seeking a method for categorizing the vegetation parts based on their exposure to the sunlight. Therefore, the pattern of relative variations in the levels of the R, G, and B triplets was evaluated from the darkest vegetation pixels towards the lightest ones. To implement this idea, the vegetation pixels were categorized into the maximum possible number of 255 groups based on their green levels (i.e. in the range of 0-255, RGB color system). Subsequently, the averaged red and averaged blue levels of each group were calculated, and the total sets of the results were plotted against the green ascending sequence. It was observed that the two sets of the red and blue mean values yielded two smooth upward exponential shaped curves, among which the blue one had apparently a higher degree of curvature compared with red (more detail in the Results section). Since the classification of vegetation pixels (as a kind of image segmentation) were based on the ascending green levels, we named the method GSM (green-gradient based segmentation method).

Considering the simplicity of the method in both implementation and interpretation, and also several interesting aspects revealed by further assessments, it appeared that GSM might be developed as a relatively efficient computation platform in canopy image analyses. Therefore, the purpose of the present study was evaluating the option of developing GSM as an image-based descriptive platform for canopy studies, and also as a source of efficacious vegetation criteria in high-throughput phenotyping approaches. 


\section{Materials and Methods}

\subsection{Field experiment}

The field experiment was conducted at the research field of School of Agriculture, Shiraz University, Iran $\left(29^{\circ} 73^{\prime} \mathrm{N}\right.$ latitude and $52^{\circ} 59^{\prime} \mathrm{E}$ longitude at an altitude of 1,810 masl) during the 2014-15 and 2015-16 growing seasons. The experiment consisted of $90(2 \times 2$ meter) plots including every 15 monocultures and mixtures of four early to middle ripening wheat cultivars under two normal and post-anthesis deficit irrigation conditions in 3 replicates (so that there were 6 replicates available at the time of imaging i.e. booting phase). The experimental design was RCBD (Randomized Complete Block Design) in a lattice arrangement with 1 meter distances between the plots. The early to middle ripening wheat cultivars used were Chamran (1), Sirvan (2), Pishtaz (3), and Shiraz (4), respectively. For providing mixtures, seeds of the cultivars were mixed before sowing on flat beds, with equal ratios based on their 1000-grain weights, germination percentages, and the fixed planting density of 450 plants per square meter. The planting dates in the first and second growing seasons were November 20 and November 5, respectively. Depending on soil test, only nitrogen fertilizer (urea) was applied, 3 times i.e. in planting, early tillering, and anthesis, with a total amount of $150 \mathrm{~kg} \mathrm{~N} / \mathrm{ha}$. No chemical was used, and weeding was done by hand.

Irrigation interval was 10 days based on local practices, and the amount of irrigation water was estimated using the Fao-56 Penman-Monteith model with local corrected coefficients which was reduced to $50 \%$ of evapo-transpirational demand from the first irrigation after anthesis (Haghshenas and Emam, 2019). Late in the season, plants were harvested from the center of plots (equal to the overall row length of $3 \mathrm{~m}$, or $0.6 \mathrm{~m}^{2}$ per plot) and grain yield was calculated using a laboratory thresher and weighing.

\subsection{Imaging}

The nadir images were taken at late booting stage i.e. 155 DAS (days after sowing; April 24,2015 ) from $150 \mathrm{~cm}$ above the soil surface (about one meter above the top of the canopy). At this growth stage, wheat stand can provide a desirable typical canopy, i.e. a sufficiently dense canopy (most leaves are fully emerged and still thoroughly green); moreover, spikes are not emerged yet (which also might be included in analyses as green organs otherwise). We used a common commercial digital camera (Canon PowerShot SX100 IS) which was set to auto mode and the maximum imaging resolution of 8.0 megapixels. 
Images used in the phenology and stress evaluations were selected from the second season's archive (2015-16, due to occurrence of the cold stress, and more frequent imaging practices continued until the canopy dried completely).

\subsection{Image processing and data analyses}

\subsubsection{Segmentations type 1, and type 2 (i.e. GSM)}

Image processing was carried out using an exclusive MATLAB code (MATLAB 2015b). Following a common segmentation of each image into vegetation and background parts (segmentation type 1, $\mathrm{ST}_{1}$ ) using the thresholding formula of G-R>0 (Wang et al., 2016; G and $\mathrm{R}$ stand for green and red color values in RGB color system, respectively), pixels of vegetation parts were categorized into the maximum possible number of 255 groups, based on their similar green values (segmentation type $2, \mathrm{ST}_{2}$; RGB color system). Then, the averaged red and averaged blue values of each group were calculated, and subsequently their separate trends were plotted against the green sequence.

In $\mathrm{ST}_{2}$, the level of green triplet was selected as the basic exposure criterion, since although we had red, blue, grayscale, and other criteria as alternatives, the green band has obviously the most rigorous reflection from vegetation, which is expected to provide the most robust signal and also determines the exposure status more accurately, compared with the red and blue colors. In addition, it is directly a record of the light intensity in a given band collected by the sensor, without any conversion, despite the other formula-based indices e.g. the synthesized grayscale. Below is the mathematical representation of GSM (i.e. $\mathrm{ST}_{2}$ ):

$$
\begin{aligned}
& G:\{1,2, \ldots, 255\} \rightarrow\{1,2, \ldots, 255\} \\
& i \mapsto G_{i} \\
& \text { where } G_{i} \text { is the green level of vegetation pixels (in } R G B \text { color system) } \\
& R:\{0,1,2, \ldots, 255\} \rightarrow\{0,1,2, \ldots, 255\} \\
& i \mapsto R_{G_{i}} \\
& B:\{0,1,2, \ldots, 255\} \rightarrow\{0,1,2, \ldots, 255\} \\
& i \mapsto B_{G_{i}} \\
& \text { where } R_{G_{i}} \text { and } B_{G_{i}} \text { are the red and blue levels of pixels with } G_{i}=i . \\
& \text { Let: }
\end{aligned}
$$




$$
\begin{aligned}
& N_{G_{i}}:=\text { Number of pixels with } G_{i}=i . \\
& R_{G_{i}}:=\text { Red level of pixels with } G_{i}=i . \\
& B_{G_{i}}:=\text { Blue level of pixels with } G_{i}=i . \\
& r:\{0,1,2, \ldots, 255\} \rightarrow[0,255] \\
& i \mapsto \frac{\sum R_{G_{i}}}{N_{G_{i}}} \\
& b:\{0,1,2, \ldots, 255\} \rightarrow[0,255] \\
& i \mapsto \frac{\sum B_{G_{i}}}{N_{G_{i}}} \\
& g:\{1,2, \ldots, 255\} \rightarrow\{1,2, \ldots, 255\} \\
& i \mapsto G(i)
\end{aligned}
$$

\subsubsection{Segmentation type 3}

Comparing the local slopes (differentiations) of the GSM red and blue curves with the slope of the green line (i.e. equals 1), five apparent parts (classes) were distinguished on each of the red and blue curves, based on which the curves were segmented. This curve segmentation was called "segmentation type $3\left(\mathrm{ST}_{3}\right)$ " for avoiding confusion with the previously described segmentations. Subsequently, the vegetation parts of the respective images were segmented and evaluated. For this purpose, the extreme green levels of each ST3 curve segments were used as inputs for image segmentation. In order to monitoring the calculation processes and results carefully, $\mathrm{ST}_{3}$ was carried out and checked manually, using Origin Pro 8 software (OriginLab, Northampton, MA) and formulated Excel sheets. The algorithm followed for $\mathrm{ST}_{3}$ segmentation of the red curve was:

$$
\begin{aligned}
& m \text { and } p \in \mathbb{N} \\
& m:=20 \\
& p:=50 \\
& i_{\text {max }}: \text { the level of } i \text { at which }\left(\frac{d r}{d i}\right) \text { is maximum throgh the whole curve. }
\end{aligned}
$$


if $\left[\left(\frac{d r}{d i}<1\right) A N D\left(\frac{d r}{d(i-1)}<1\right) A N D \ldots A N D\left(\frac{d r}{d(i-m)}<1\right)\right]$ OR $\left[\left(\frac{d r}{d i}<1\right) A N D\left(\frac{d r}{d(i+1)}<\right.\right.$

1) $\left.A N D \ldots A N D\left(\frac{d r}{d(i+m)}<1\right)\right]$ :

else

$$
\begin{aligned}
& \text { if }(p<i \\
& \left.<i_{\text {max }}\right) A N D\left[\left(1 \leq \frac{d r}{d i}\right) \text { OR }\left(1 \leq \frac{d r}{d(i-1)}\right) \text { OR ... OR }\left(1 \leq \frac{d r}{d(i-m)}\right)\right] \text { AND }\left[\left(1 \leq \frac{d r}{d i}\right) \text { OR }(1\right. \\
& \left.\left.\leq \frac{d r}{d(i+1)}\right) \text { OR ... OR }\left(1 \leq \frac{d r}{d(i+m)}\right)\right] \text { AND NOT }\left[\left(1 \leq \frac{d r}{d i}\right) \text { AND }(1\right. \\
& \left.\leq \frac{d r}{d(i+1)}\right) A N D \ldots A N D(1 \\
& \left.\left.\leq \frac{d r}{d(i+m)}\right)\right] \text { : } \\
& \text { else } \\
& \text { if }\left[\left(1 \leq \frac{d r}{d i}\right) A N D\left(1 \leq \frac{d r}{d(i-1)}\right) A N D \ldots A N D\left(1 \leq \frac{d r}{d(i-m)}\right)\right] \text { OR }\left[\left(1 \leq \frac{d r}{d i}\right) A N D(1\right. \\
& \left.\leq \frac{d r}{d(i+1)}\right) A N D \ldots A N D(1 \\
& \left.\left.\leq \frac{d r}{d(i+m)}\right)\right]
\end{aligned}
$$

$$
\begin{aligned}
& \text { else } \\
& \text { if } i_{\max }<i \text { : } \\
& \text { else: }
\end{aligned}
$$$$
i \in \text { Class } V
$$

where $m$, which was here set to 20 , is the number of neighborhoods of $i$ included in the evaluation for determining the class of $i$; and $p$ is a threshold defined to distinguish the elements of the class I from the classes III and V. Moreover, $\max \left(\frac{d r}{d i}\right)$ is the maximum amount of $\left(\frac{d r}{d i}\right)$ through the whole set of results (for $i=1$ to 255). In a similar way, $\mathrm{ST}_{3}$ may be also carried out for the blue curve.

\subsubsection{GSM curve characteristics}

For assessing the option of exploiting the GSM curve characteristics as potential vegetation indices, 169 attributes were defined and analyzed for the whole curves and also for $\mathrm{ST}_{3}$ curve segments, e.g. using the coefficients of the best fitted exponential and polynomial equations to the curves, total under- and/or between curve areas (AUCs and BCAs), local and linear slopes, the relative ratios of the curve attributes, and also the number of image pixels 
assigned to each $\mathrm{ST}_{3}$ segment. The last series of attributes (i.e. the 29 attributes defined based on the number of pixels) were entitled "GSM-coverage attributes", since they indicate the percentage image area covered by each of the $\mathrm{ST}_{3}$ segments.

Table S1 represents the complete list of the curve-derived attributes and definitions. Following estimating curve differentials and integrations by Origin Pro (as represented), GSM characteristics were calculated in formulated excel sheets.

The nonlinear equations fitted to the curves, were the simple exponential and polynomial forms as shown below:

Polynomial $\left\{\begin{array}{l}r_{\text {poly }}=a_{R}\left(G_{i}{ }^{2}\right)+b_{R}\left(G_{i}\right)+c_{R} \\ b_{\text {poly }}=a_{B}\left(G_{i}{ }^{2}\right)+b_{B}\left(G_{i}\right)+c_{B}\end{array}\right.$

Exponential $\left\{\begin{array}{l}r_{\text {exp }}=a_{R} \cdot e^{b_{R} \cdot G_{i}} \\ b_{\text {exp }}=a_{B} \cdot e^{b_{B} \cdot G_{i}}\end{array}\right.$

where $r_{\text {poly }}, b_{\text {poly }}, r_{\text {exp }}$, and $b_{\text {exp }}$ are the polynomial and exponential equations for the GSM red and blue curves, $a, b, c$ are the polynomial equation constants, and $a_{R}, a_{B}, b_{R}, b_{B}$ are the exponential coefficients for GSM red and blue curves, respectively.

The Local- and linear-slopes of the GSM red and blue curves were calculated as respective differentials $\left(\frac{d r}{d g}\right.$ or $\left.\frac{d b}{d g}\right)$ and tangents $\left(\frac{\Delta R_{G_{i}}}{\Delta G_{i}}\right.$ or $\left.\frac{\Delta B_{G_{i}}}{\Delta G_{i}}\right)$ in the defined ranges. Furthermore, curve area calculations (including separate or total area under red or blue curves, and various between-curve areas) were carried out using definite integrations. For instance, total AUCs (under curve areas) for red or blue curves were estimated by:

$$
\begin{aligned}
& A U C_{r}=\int_{G_{1}}^{G_{255}}\left(a_{R} \cdot e^{b_{R} \cdot G_{i}}\right) d g \\
& A U C_{b}=\int_{G_{1}}^{G_{255}}\left(a_{B} \cdot e^{b_{B} \cdot G}\right) d g
\end{aligned}
$$

Moreover, the total BCAs (between-curve areas) of $g-r, g-b$, and $r-b$ were determined as:

$$
\begin{aligned}
& B C A_{G-R}=\int_{G_{1}}^{G_{255}}\left(a_{G} \cdot G_{i}+b_{G}\right) d g-\int_{G_{1}}^{G_{255}}\left(a_{R} \cdot e^{b_{R} \cdot G_{i}}\right) d g \\
& B C A_{G-B}=\int_{G_{1}}^{G_{255}}\left(a_{G} \cdot G_{i}+b_{G}\right) d g-\int_{G_{1}}^{G_{255}}\left(a_{B} \cdot e^{b_{B} \cdot G_{i}}\right) d g
\end{aligned}
$$


where $a_{G}$ and $b_{G}$ are the coefficient and the constant for the linear equation $g$ trend; and:

$$
B C A_{R-B}=\int_{G_{1}}^{G_{255}}\left(a_{R} \cdot e^{b_{R} \cdot G_{i}}\right) d g-\int_{G_{1}}^{G_{255}}\left(a_{B} \cdot e^{b_{B} \cdot G_{i}}\right) d g
$$

The definite integrals for every distinct curve segments $\left(\mathrm{ST}_{3}\right)$ were also calculated in a similar way.

In order to explore the potential relationships between various GSM-curve derived attributes and grain yield $(\mathrm{GY})$ or number of grains $\mathrm{m}^{-2}(\mathrm{GN})$, some data mining techniques were used including Decision trees, Deep learning, General linear model, Gradient boosted trees, ID3, Neural networks, Random Forest, and also weight models (analyses were carried out using RapidMiner Studio version 8.1). Accordingly, the option of GY and GN predictions based on GSM curve attributes was also evaluated. Based on the type of data preparation, the efficacy of the attributes was assessed in 3 different computation and prediction approaches:

$I^{\text {st }}$ approach: in this approach, all the values of the GSM attributes (as the independent factors), and GY or GN (as the dependent factor) were quantitative (numerical);

$2^{\text {nd }}$ approach: the values of GSM attributes were quantitative, but the GY or GN values were separately divided into two qualitative (binominal) classes of "high yielding/ or grain numbers" and "low yielding/ or grain numbers" using K-means clustering; and

$3^{\text {rd }}$ approach: all the values of GSM attributes and also GY or GN were separately classified in various qualitative (polynominal) groups using Agglomerative Hierarchical Clustering (AHC, using XLSTAT Version 2016.02.28451 -Addinsoft-; moreover, the clustering criterion was Euclidian distance and the number of groups for each variable was also determined by the AHC itself). Before running AHC, values of the intended variable were individually sorted in ascending order (otherwise, different results might be achieved from different runs of the clustering). Results of AHC are provided in Table S1.

Statistical analyses were carried out using IBM SPSS Statistics for Windows, Version 19.0 (Armonk, NY: IBM Corp.) and all mean comparisons were performed by Tukey's test. Comparison and reconciliation between images and the $\mathrm{ST}_{3}$-derived graph segments were performed using Fiji and MATLAB, and some casual equation evaluations were carried out by XLSTAT. For keeping consistency among the figures, everywhere an identical image and 
its GSM graph (which was captured from the experimental plot of the monoculture of the $1^{\text {st }}$ cultivar in the second replicate) was used, unless mentioned otherwise.

\section{Results}

\subsection{The overall form of $G S M\left(S T_{2}\right)$}

As shown in Fig. 1A \& B, in the GSM graph of wheat canopy, the variation trends of mean red and mean blue values relative to the 1:1 green line, formed two smooth upward exponential shaped curves, among which the blue curve had a higher degree of curvature. Indeed, similar pattern could be observed for the images of all plots. The red and blue curves showed robust fit to exponential and polynomial equations. The comparison carried out between the exponential and polynomial types of curve fittings for 24 images, taken from the monoculture plots (in 6 replicates), revealed that the exponential equation of the blue curve had higher fitness, compared to the red curve, while the fitness of polynomial equations of the both curves were almost similar. However, the coefficients of the exponential equations of both red and blue curves showed higher stabilities, compared with the coefficients of polynomial equations (Fig S1).

\subsection{Segmentation type $3\left(S T_{3}\right)$}

Evaluating the comparative local slopes (differentiations) of the red and blue trends relative to the 1:1 green line, 5 apparent parts (classes) on either red or blue curves were distinguished, based on which, consequently another type of segmentation was carried out (ST3, i.e. GSM curve-based segmentation, Fig. 1C). Also reconciling these curve-derived classes to the images by thresholding (Fig. 1C-E \& Fig. S2) and visual assessments, five distinct corresponding segments were recognized in the vegetation parts of the images, as described below (from the lightest pixels towards the darkest ones):

- The highly reflective sun-exposed vegetation surfaces i.e. include the lightest pixels of the green canopy due to their spatial configurations relative to the sunlight and camera (based on bidirectional reflectance distribution function, BDRF). In this image segment, angles of the green surfaces are relatively homogeneous and show low variations. On the GSM curve, the red or blue trends in the range of this $\mathrm{ST}_{3}$ class, has non-stable local slopes greater than 1, which eventually at the left side extreme, approach the maximum declining rate (highest local slope) of the entire curve. This $\mathrm{ST}_{3}$ class is named "Class 

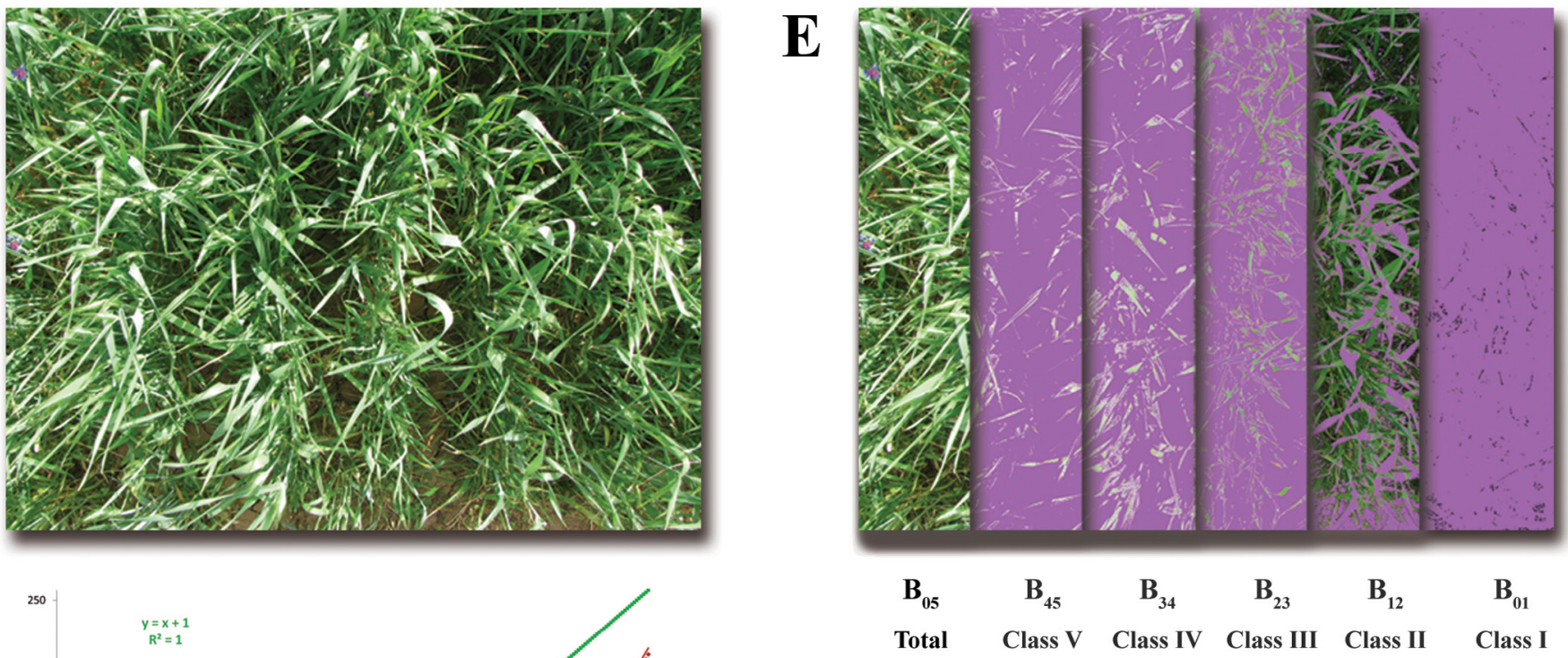

B

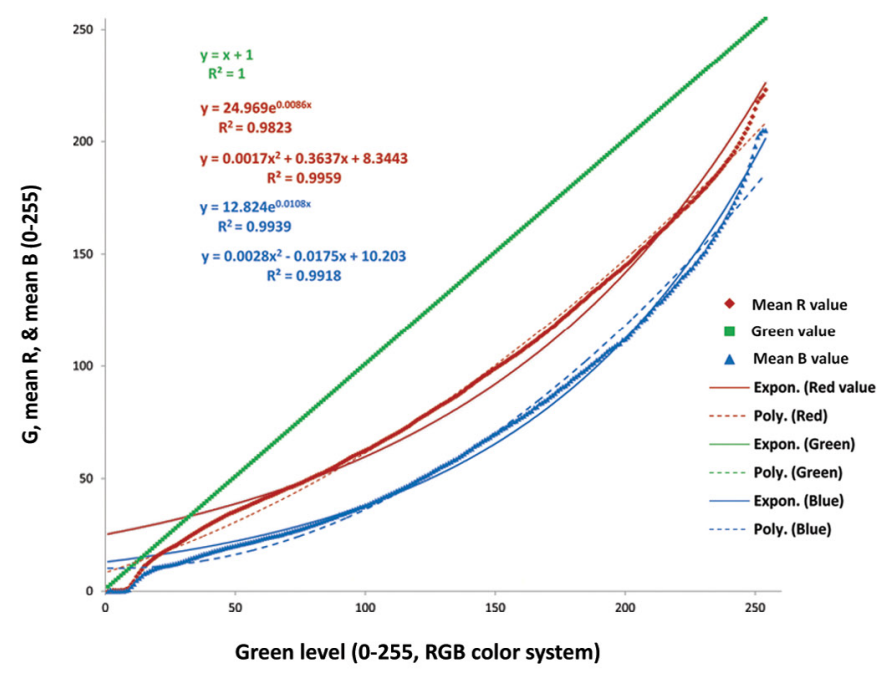

Total Class V Class IV Class III Class II Class I

(ST 3 Segments; B: blue)

Figure 1. Illustration of green-gradient based canopy segmentation (GSM) and the GSM-curve derived segmentation type $3\left(\mathrm{ST}_{3}\right)$. (A) the original image taken from monoculture of the1st wheat cultivar. All of the graphs in other parts are resulted from processing this image. (B) Canopy GSM graph. The trend lines of the best polynomial and exponential equations fitted on the red and blue curves are shown. (C) The relative variations in local slopes of the red and blue GSM curves, compared to the green line. $\mathrm{ST}_{3}$ is developed based on this graph; see the horizontal red and blue bars below the graph, which are divided into 5 ST3 classes. (D) Segmentation type 3 (i.e. the GSM-curve segmentation based on status of variations in local slopes compared to the green trend). The set of five vertical color bars at the left side show the comparative theoretical and actual color variations. The color bars are constructed using the real outputs of GSM, as the "RGB" bar is made using the overall mean of red, green, and blue values at each green level; the "Obs. R" and "Obs. B" bars indicate the actual attenuating trend of red and blue colors in the GSM graph, respectively; and the " $1: 1 \mathrm{R}$ " and " $1: 1 \mathrm{~B}$ " bars show the conceptual attenuating trends of the red and blue colors, if they had declined linearly as same as the green line. (E) The result of image segmentation based on $\mathrm{ST}_{3}$ segmentation of the GSM blue curve. 
$V$,, and the related curve range of the red and blue trends are represented as $\operatorname{Red}_{45}$ and Blue 45 , respectively.

- The image segment which covers all of the remained sun-exposed green surfaces with a complete set of altering angles. On the curve segment (i.e. Class IV, with the curve range of $\operatorname{Red}_{34}$ /or Blue 34 ), the sharpest declining slope of the GSM curves begins to decrease regularly towards the another threshold at which the local slope reaches that of the green line i.e. 1.

- The segment of partial shadow (class III, with the curve range of $\operatorname{Red}_{23}$ /or Blue 23 ): Based on the visual assessments, this image segment seems to match the vegetation surfaces positioned under partial shadow (penumbra, Figs. 1 \& S2). The GSM red and blue curves experience frequent fluctuations in relative local slopes around 1 (repeated slope shifting above and below the rate of the green line). It may imply that under partial shadow, the spectral behavior of vegetation in the red and blue bands are almost similar to the green one.

- The segment of complete shadow (class II, with the curve range of $\operatorname{Red}_{12}$ /or Blue 12 ): this image segment includes the vegetation surfaces casted by shadow, i.e. lacks any sun-exposed point. Here, the green color of the vegetation surfaces is visually recognizable. This curve segment is the widest one across the GSM curve (includes the most diverse green values on the horizontal axis) with steadily declining relative slopes less than 1, i.e. has less reductions in the level of the red and blue colors compared with green (this trend may also be comparable with the reported evidences for further penetration of green light into the leaf, as a particular micro-canopy; Sun et al., 1998; Terashima et al., 2009).

- The segment of deep shadow (class $\mathrm{I}$, with the curve range of $\mathrm{R}_{01}$ or $\mathrm{B}_{01}$ ): this image segment is composed of the darkest green pixels of the canopy in the image. On the GSM curve, the local slopes of red and blue trends exceed again the constant slope of 1 (i.e. that of the green linear trend).). Finally, blue light intensity reaches a nondetectable reflection earlier than red on the curve, i.e. maybe less penetration to the deepest parts of the canopy.

Remarkably, the five $\mathrm{ST}_{3}$-derived classes described are complementary to each other (both in the image and on the curve) and therefore, their integration cover all the vegetation pixels in the image (includes the entire crop coverage -CC-). Moreover, while the separate implementation of the $\mathrm{ST}_{3}$ segmentation on the red and blue curves yielded a highly 
overlapped curve- and/or image-segments, yet, there were frequent finely differences between them, which indicates that the shading pattern (and thresholds) is a band depended concept and at the range extremes, shows minor dissimilarities based on the different colors assessed. This evidence, together with the precise differentiation of the canopy pixels in the class III and II (penumbra vs. complete shadow, Fig. S2), may indicate the relative efficacy of the $\mathrm{ST}_{3}$ segmentation in shading pattern evaluations.

Figures 2 and S3 represent some aspects of GSM revealed using analyses of the GSM curve-characteristics, most of which are based on the $\mathrm{ST}_{3}$ segmentation. Accordingly, several observations are described below:

- For each of the red or blue curves, there was a strong negative correlation between the coefficients ( $a$ and $b$ ) of the fitted exponential equation (Fig. S3 F\&G).

- Comparing the linear slopes of red and blue curves in the $\mathrm{ST}_{3}$ classes IV \& V (which were jointly assigned to the total sun-exposed parts), the highest declining rates were observed oppositely in different classes (Fig. S3 C); as the linear slope of the red curve in the class V was higher compared with that in the class IV (i.e. in the segment with more slanted towards relatively vertical surfaces); while, the linear decreasing rate of the blue curve in class IV is relatively higher than in class V. It may be another evidence for different behavior of red and blue bands, which is expected to be mainly associated with the variations in their BDRF reflectance in response to the altering angles of vegetation surfaces.

- Interestingly, the 15 mixture treatments applied, significantly affected the G-R and G-B BCAs (between-curve areas) in the $\mathrm{ST}_{3}$ class II (i.e. the image segment of complete shadow) while, generally such differences were not observed in other segments (except for $\operatorname{Red}_{45}$ ). It may be an evidence for different responses of wheat genotypes and/or their mixtures to shading (whereas no shading treatment was applied).

- On each of the red or blue curves, the linear slope of the curve in the class II (the segment of complete shadow) was almost equal to the inversed rate of the similar slope in the class IV (Fig. 2 C). However, this observation seems to be limited to the present study (which is focused on analyzing the images taken at wheat booting phase of the stand grown under almost normal conditions), and further extrapolations require more investigations. 
A
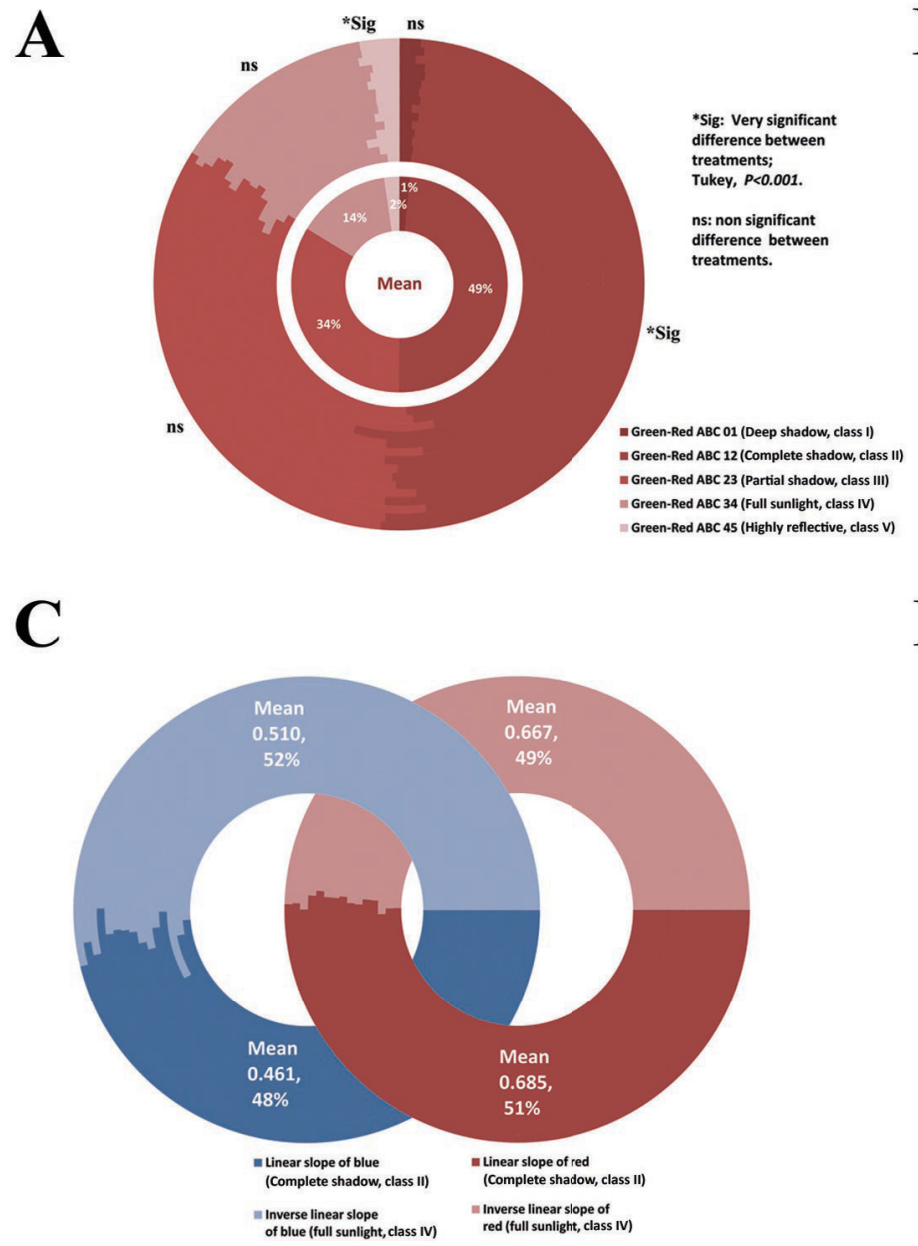

Figure 2. Several aspects of GSM revealed evaluating the GSM-curve characteristics. (A). The area between the green trend and red GSM curve as influenced by the 15 mixture treatments/90 plots. (B) The area between the green trend and blue GSM curve as influenced by the 15 mixture treatments/90 plots. (C) In the dense canopy of wheat booting phase, the rate (linear slope) of the red or blue GSM curve in the segments assigned to shadow is almost equal to its inverse rate of the full sunlight segment. In the doughnut charts of sections A to C: the 15 mixture treatments (i.e. 1, 2, 3, 4, 12, 13, 14, 23, 24, 34, $123,124,134,234$, and 1234) are arranged from center towards outside, respectively. "1" to " 4 " represent the early to middle ripening wheat cultivars, and each digit is a cultivar included in the mixture. (D) The x-lengths of the GSM ST 3 classes (shadow to light segments) of the red and blue curves (excluding the deep shadow segment, class I). (E) The percentages of canopy coverage assigned to each $\mathrm{ST}_{3}$ segment. Similar to section $\mathrm{D}$, the ratios follow exponential trends. The charts in the sections $\mathrm{D}$ and $\mathrm{E}$ are drawn using the overall mean values of 90 plots, while a similar trend was also observed for each individual plot.
B

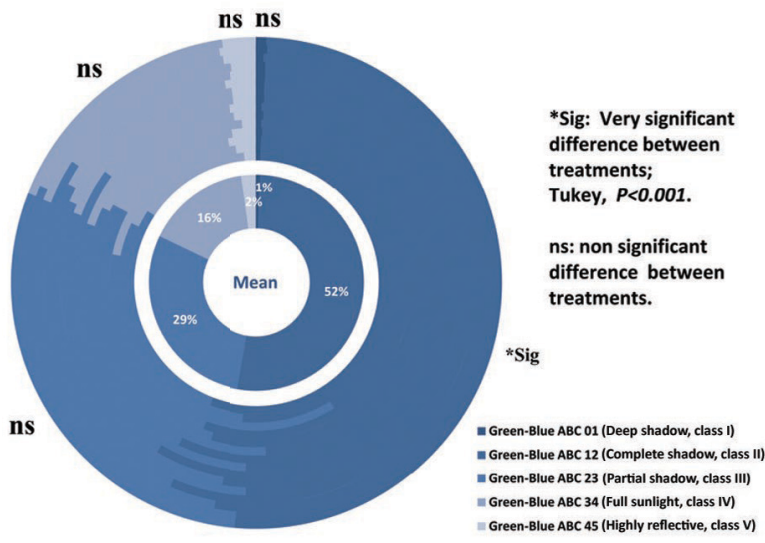

D

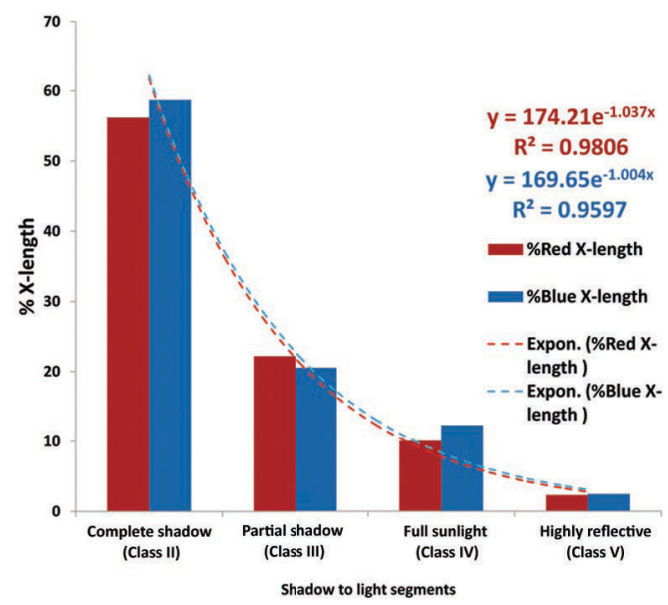

E

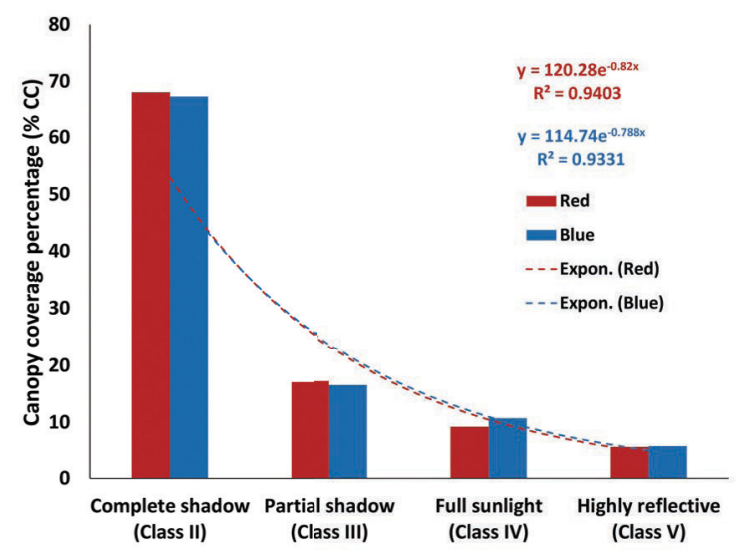


- The ascending series of canopy coverage percentages of each $\mathrm{ST}_{3}$ class, i.e. $\{\% \mathrm{Num}$ $B_{12} / C C$, \%Num $B_{23} / C C$, \%Num $B_{34} / C C$, and \%Num $\left.B_{45} / C C\right\}$ or $\left\{\% N u m R_{12} / C C, \%\right.$ Num $R_{23} / C C$, \%Num $R_{34} / C C$, and \%Num $R_{45} / C C$ \} follow individual exponential trends. In other words, there was an exponential relationship among the ratios of canopy coverage assigned to the complete shadow (ST 3 class II), partial shadow (class III), full sunlight (class IV), and highly reflective (class V) segments, respectively (see Fig. 2 parts E and also $\mathrm{D}$, which are in contribution with each other; since the $\mathrm{ST}_{3}$ image segmentation was based on the X-lengths of the GSM curve segments). Indeed, this observation indicated that under conditions of the present study, number of vegetation pixels exposed to complete shadow, declines exponentially towards the partial shadow, full sunlight, and eventually, the highly reflective part. However, again, it should be noted that any extrapolation to other situations requires further studies.

Figure S3 shows the schematic representation of defined GSM curve characteristics, and also some other related aspects revealed.

\subsection{The relationship between GSM-curve characteristics and grain yield and /or number} of grains $m^{-2}$

In order to evaluate the capability of the GSM-curve characteristics to be used as HTP indices, their potential relationships with grain yield and number of grains $\mathrm{m}^{-2}$ were assessed using statistical and datamining approaches. As explained before, half of the experimental plots were later subjected to post-anthesis deficit irrigation based on the other purposes of the field experiment (Haghshenas and Emam, 2019). Therefore, it had to be determined that either the data of the 45 well-irrigated plots, or the total number of 90 plots would be included in the GY and GN analyses. Indeed, it was a tradeoff between keeping the higher homogeneity among the GY or GN values of the samples, and having a higher number of observations (as much as 2 fold). As a pre-evaluation, analyses of this part (i.e. section 3.3.) were carried out in parallel using both datasets (including either 90 or 45 samples). The results showed that running the models and analyses using the 45-smaple dataset provides worse outcomes compared to the 90-sample one (data not shown). Thus, here the results of the evaluations performed using the entire 90 plots are reported. Figure S4 represents more details about the significant effects of deficit-irrigation on GY and GN, by which their values were reduced in average by $13.5 \%$ and $7 \%$, respectively. The comparison between these amounts of reduction, and a duplicated number of observations made available in the 90sample dataset may be the reason for the relative advantage of using this dataset in analyses. 
None of the individual pairwise correlations between GY or GN with the GSM-curve attributes were strong, and their correlation $\mathrm{R}$ values ranged between -0.30 to 0.28 for $\mathrm{GY}$ (Table S2) and -0.34 to 0.33 for GN (Table S3). Therefore, the potential relationships and meaningful patterns between GY or GN with combinations of the GSM attributes were sought using datamining methods.

Figure 3 shows the decision (regression) trees resulted from the $1^{\text {st }}$ computation approach (described in M\&M). Based on the rules, the curve attribute " $B_{23} A U C$ " (i.e. the under-curvearea of the blue curve in the range of $\mathrm{ST}_{3}$ segment of class III, which is shown to be associated with partial shadow) was the root node (the most determining GSM characteristic) both for GY and GN. This computation model suggests the optimal amounts of $B_{23} A U C$ (i.e. 3924.167) and " $R_{12}$ Slope/R $R_{12}$ Slope" (>1.008) which support higher GY. Similar optimal value of B23 AUC was also resulted for GN; however, attributes on the other nodes were different ones compared with GY.

In the case where the GY and GN values were separately divided into the two qualitative "high" and "low" classes (i.e. the $2^{\text {nd }}$ approach; Fig. 4), the GY and GN decision trees were similar in the first three nodes (and also in two other nodes later in the route). This may be an evidence for the accurate performance of GSM curve characteristics in recognizing more or less similar patterns for clustering GY and its major physiological contributor i.e. GN, even though here the task was as simple as distinguishing between only two classes of qualities. In this computation approach, there was one vital attribute in each of the GY and GN trees, that merely determined the class of the samples. In the GY tree, higher values of "\%Num $B_{12} C C$ " (>\%55.29) at the last node, covered all of the 56 low yielding samples; and in the GN tree, all of the low yielding samples were fell in a single subset with " $R_{34}$ slopes" greater than 1.357 .

Finally, the $3^{\text {rd }}$ approach yielded a clear categorizing of the samples based on the contribution of the key attributes "\%Num $R_{23} /$ unit of area" and " $G B_{12} A B C$ " with GY and GN, respectively (Fig. 5). Apparently the main branch of the GY tree that includes the lowest class of \%Num $R_{23} /$ unit of area, covers every high-yielding nodes (Fig. 5A). This implies that the wheat canopies whose $2 \mathrm{D}$ nadir images have higher coverage ratios of the $\mathrm{ST}_{3}$ segment of $R_{23}$ (i.e. include comparatively higher partial shadow coverages based on the red curve), were the low-yielding stands. In the another decision tree (Fig. 5B), $G B_{12} A B C$ was a supportive attribute for GN, as the two branches with the highest qualities originated from the respective 
A Grain yield (Mg/ha)
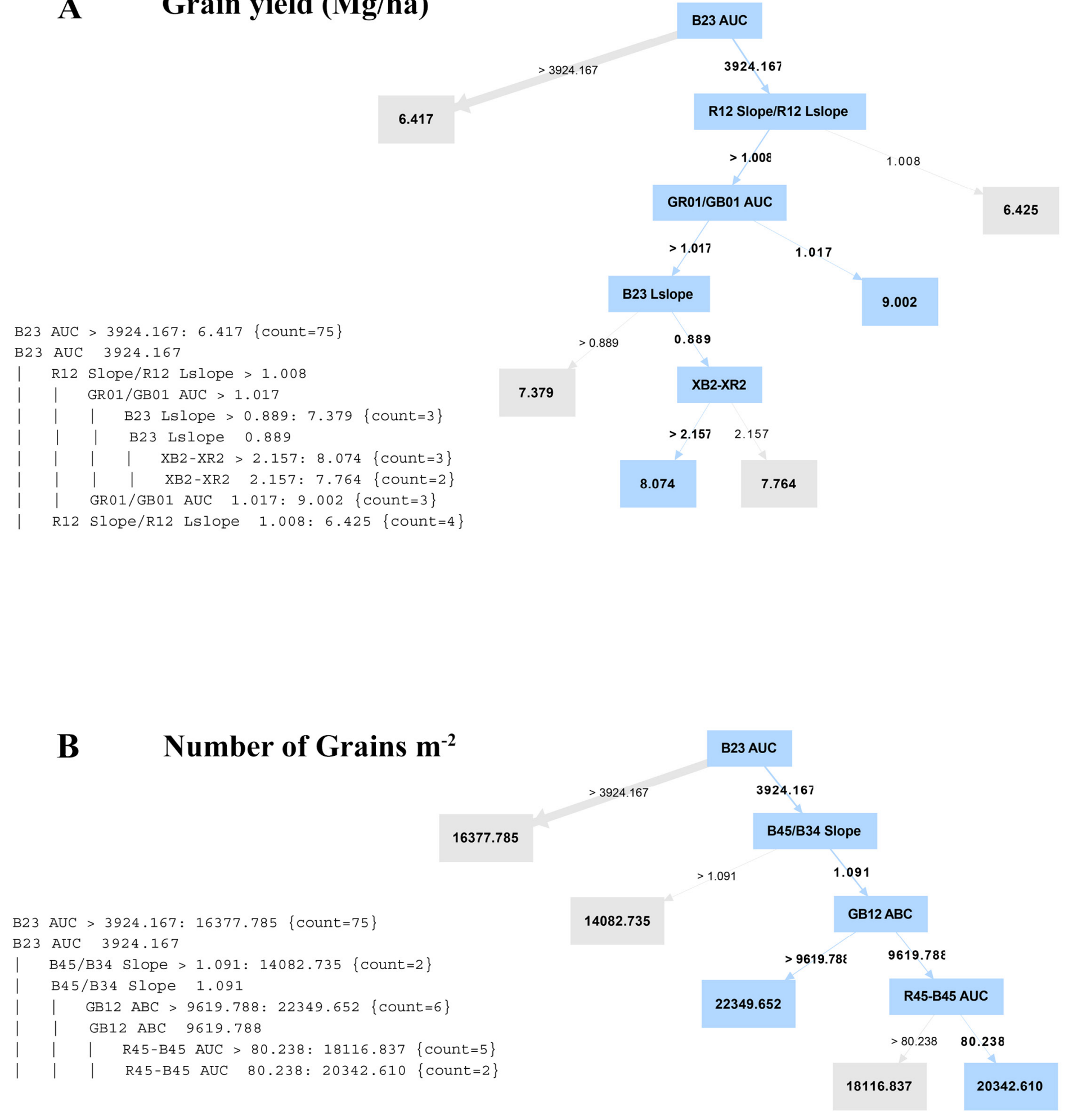

Figure 3. Decision trees for the relationships between GSM curve attributes and (A) grain yield (GY), or (B) No. of grains $\mathrm{m}^{-2}(\mathrm{GN})$. Estimations are carried out using the $1^{\text {st }}$ computation approach (based on the quantitative values, see the manuscript). The highlighted routes show the conditions for achieving the highest values of GY and GN. 
bioRxiv preprint doi: https://doi.org/10.1101/241786; this version posted January 13, 2019. The copyright holder for this preprint (which was not certified by peer review) is the author/funder. All rights reserved. No reuse allowed without permission.

\section{A \\ Grain yield (Mg/ha)}

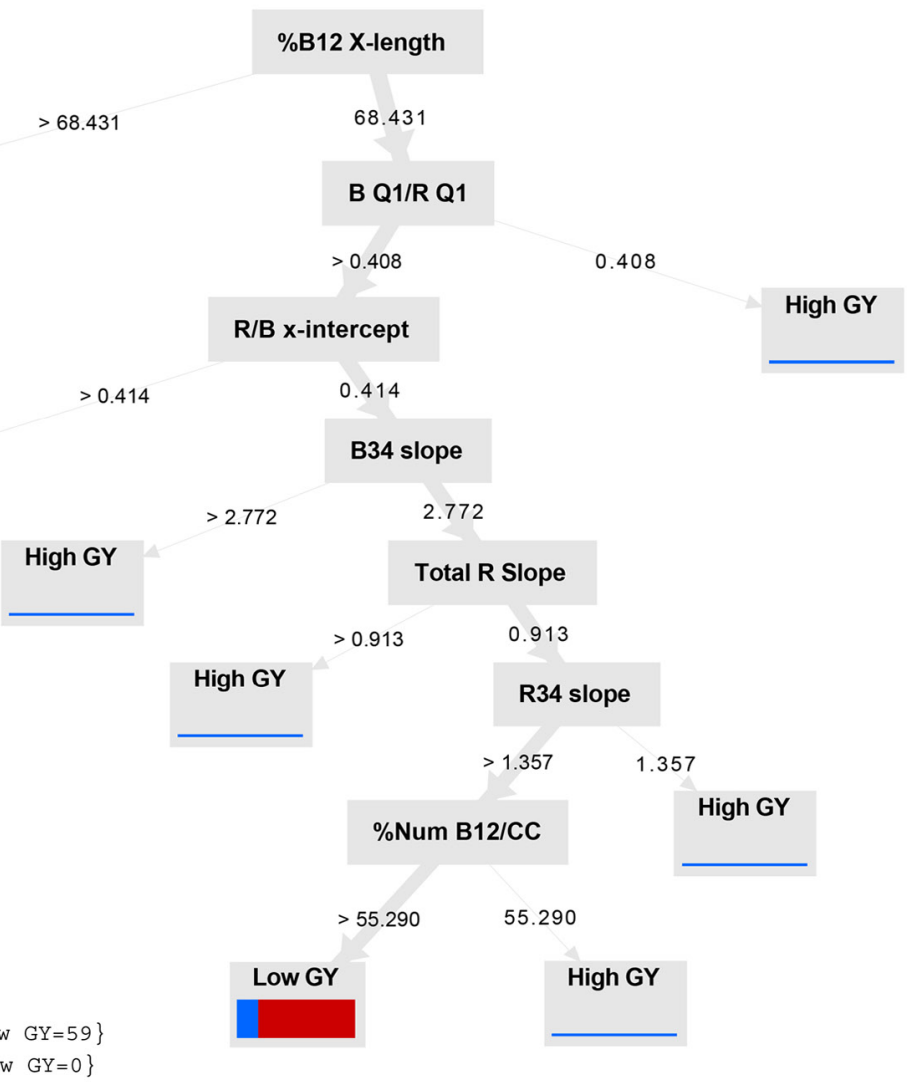

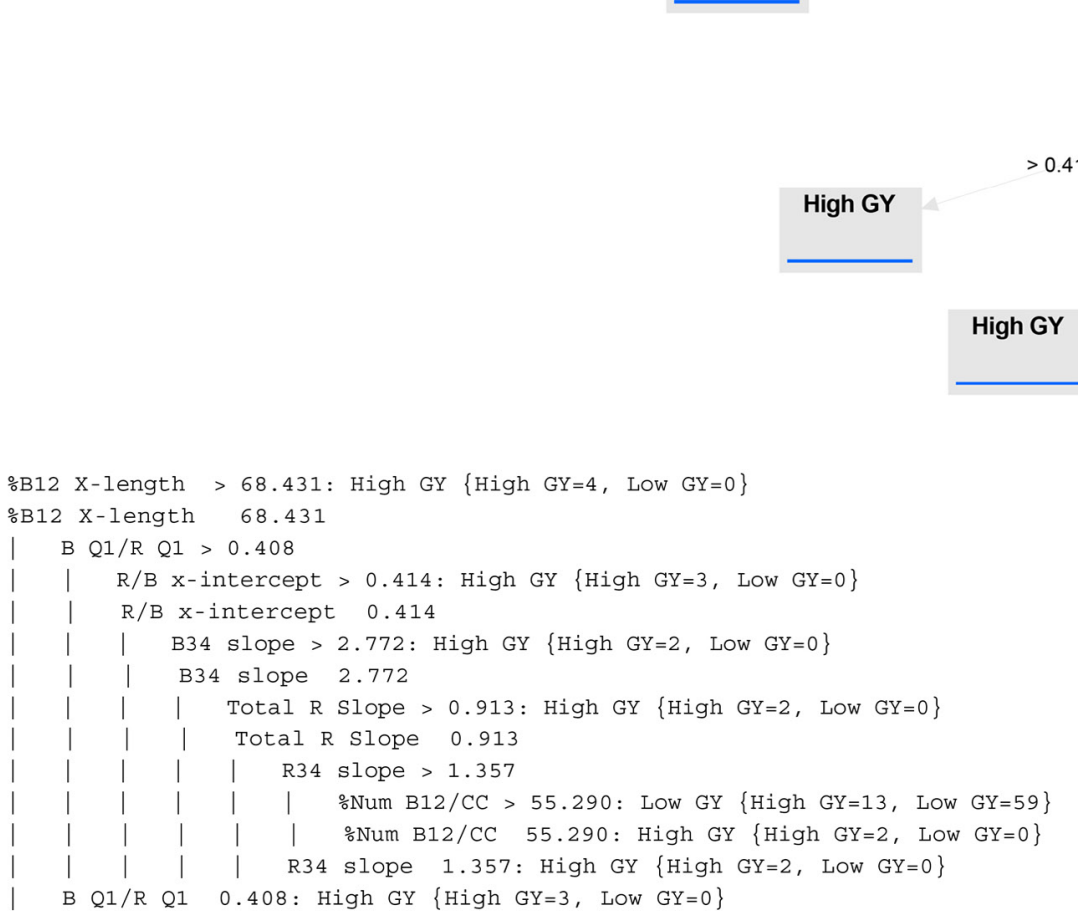

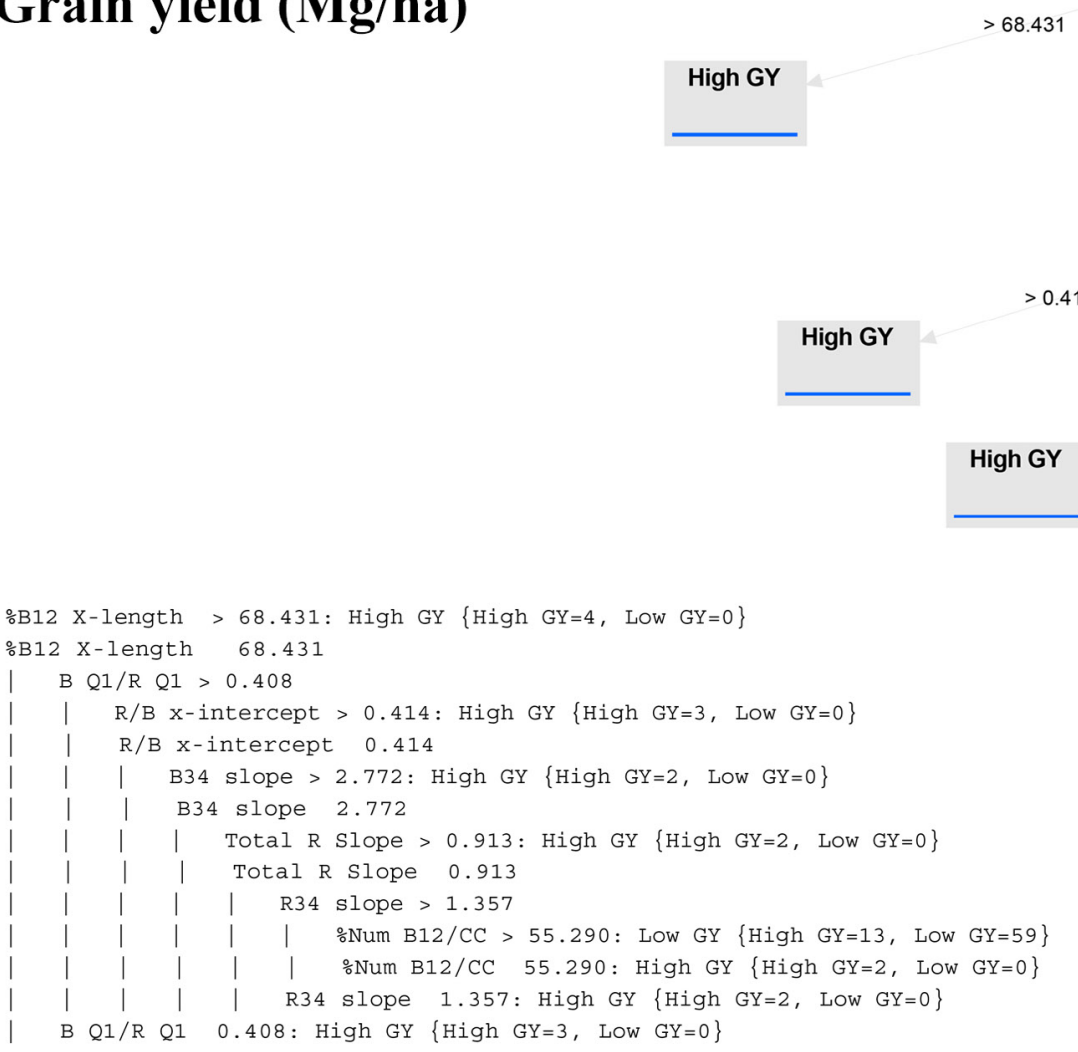

\section{B Q1/R Q1}

B

\section{Number of grains $\mathbf{m}^{-2}$}
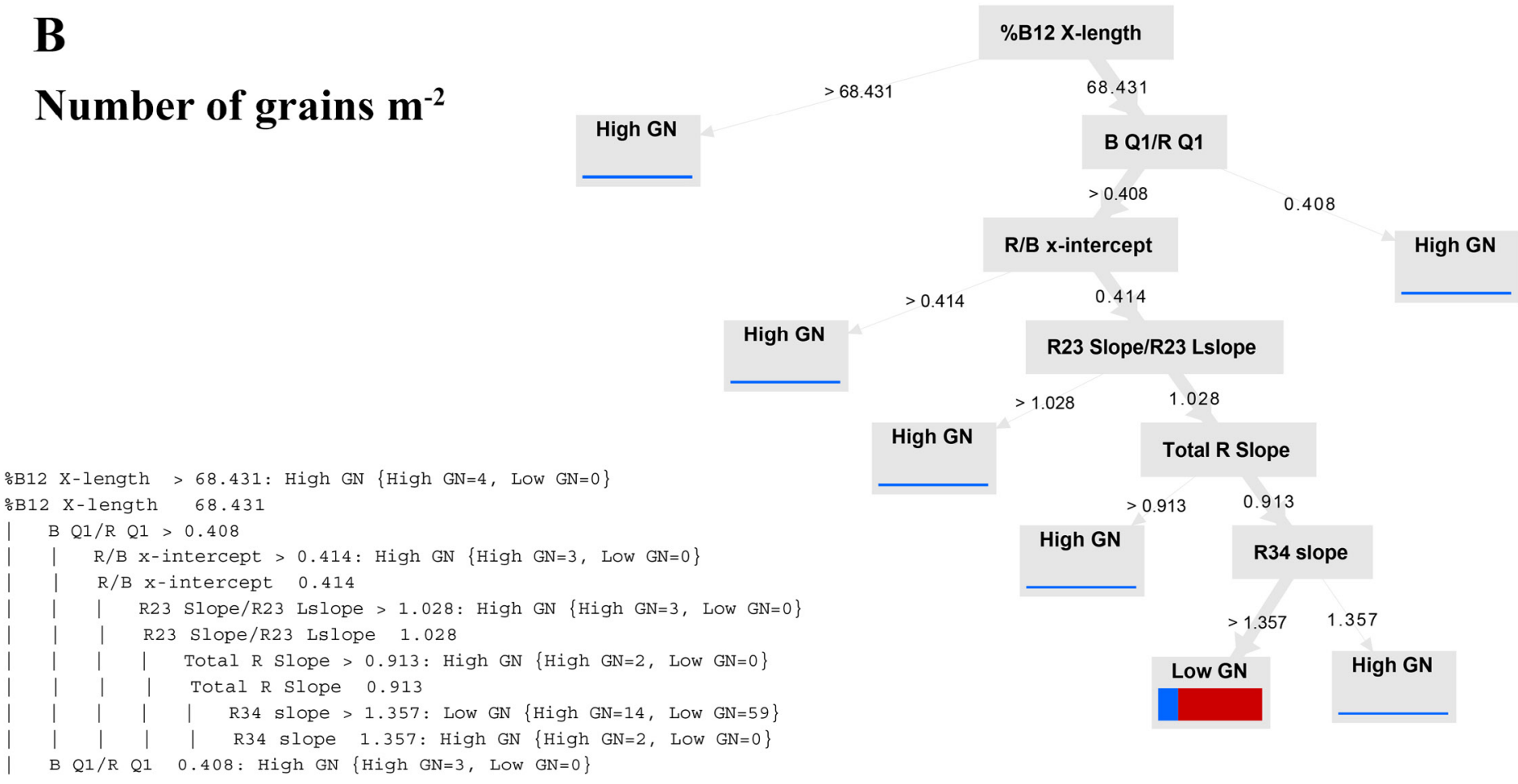

Figure 4. Decision trees for the relationships between GSM curve attributes and (A) grain yield (GY), or (B) No. of grains $\mathrm{m}^{-2}(\mathrm{GN})$. Estimations are carried out using the $2^{\text {nd }}$ computation approach (based on 2 qualitative classes of GY and GN; see the manuscript). 


\section{Grain yield (Mg/ha)}

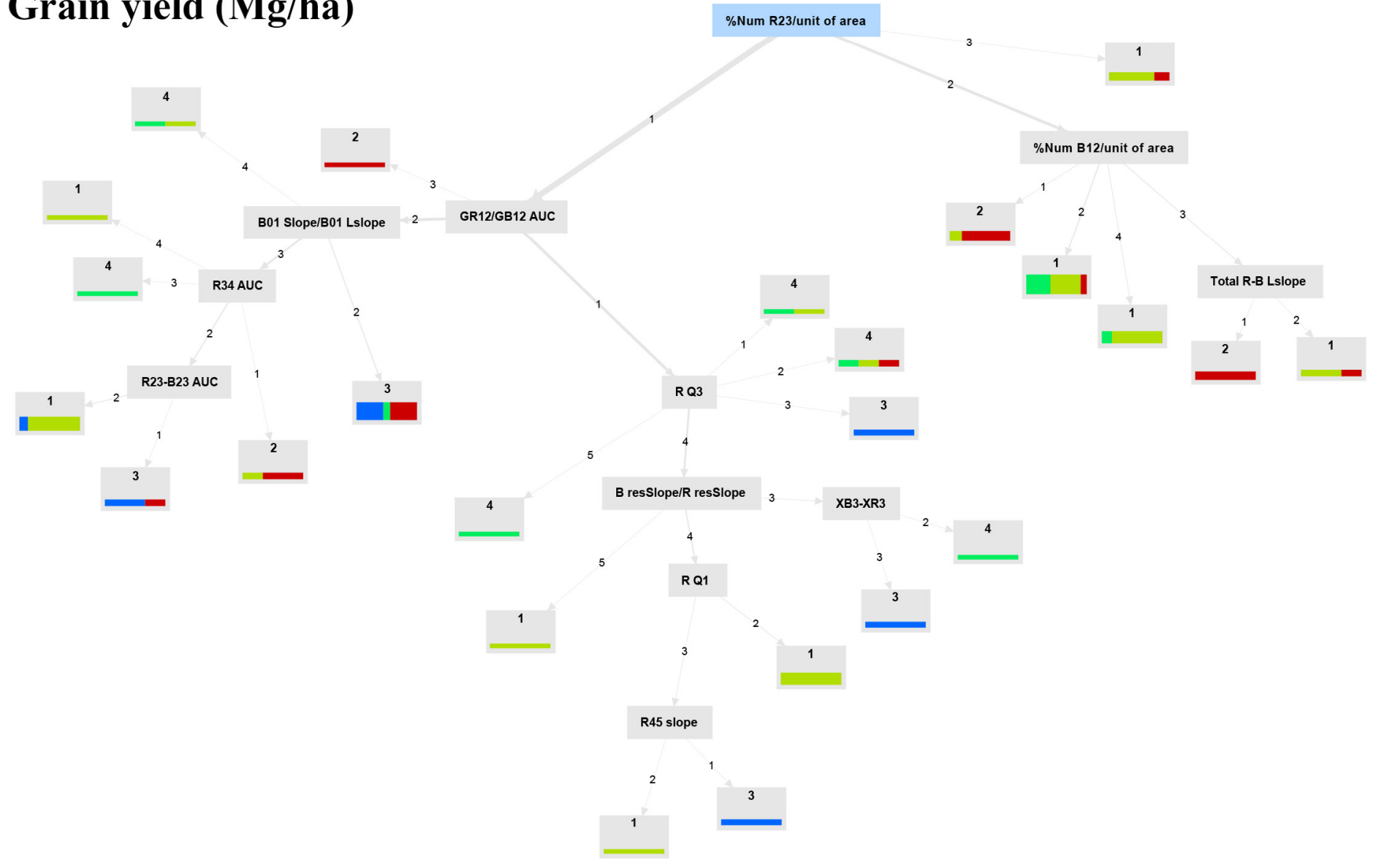

B

Number of grains $\mathbf{m}^{-2}$

Figure 5. Decision trees for the relationships between GSM curve attributes and (A) grain yield (GY), or (B) No. of grains $\mathrm{m}^{-2}(\mathrm{GN})$. Estimations are carried out using the $3^{\text {rd }}$ computation approach (based on several qualitative classes, see the manuscript, and also Table S1 for No. of the qualitative classes). Terminal (colorful) nodes indicate the classes of GY or GN. Everywhere on the chart, bigger values show the highest qualitative classes i.e. basically had higher quantitative values before clustering by Agglomerative hierarchical clustering (AHC). 
node (i.e. the root node), covered every top GN node. This may be an evidence for contribution of the complete shadow segment in GN estimations.

Besides, the relative weights of the GSM-curve attributes in determining GY and GN were estimated using 10 weight models. Based on the overall result of the weight models, the attributes "\%Num $B_{01} /$ unit of area" and " $B_{02} / B_{04} A U C$ " were suggested to have the highest contributions with GY (Table 1). Also the superior attribute for GN was "B23 Slope / B23 Lslope" (Table 2).

In addition to determining the relationships between the GSM-curve derived attributes and GY or GN, the option of GSM-based prediction of these two ultimate physiological traits using data mining models was evaluated. Predictions made by the $1^{\text {st }}$ approach analyses were not satisfactory, particularly towards the upper and lower GY and/or GN extremes. Tables 3 and 4 shows the model performance criteria, among which the minimum values of RMSE (root mean square error) for predictions of GY and GN were $1.249 \mathrm{Mg} / \mathrm{ha}$ and 3253.8 grains $\mathrm{m}^{-2}$, respectively (both of which were resulted from the predictions by the Random Forest model). Figures S5 and S6 represent the standardized residuals of the predictions by various models.

In contrast, utilizing the $2^{\text {nd }}$ and $3^{\text {rd }}$ prediction approaches provided very high to complete prediction accuracies. It is notable again that in these two approaches, the models predict the qualitative classes of GY or GN, rather than their exact quantities. As shown in Table 5 (i.e. for the $2^{\text {nd }}$ prediction approach), three out of the 6 models predicted the classes of GY with $100 \%$ accuracy, and the mean overall accuracy was about $96 \%$. Moreover, all of the samples in the lower-yielding class were recognized correctly, and the errors were in GY prediction of the higher-yielding canopies. Similarly, two models had 100\% accuracy in predicting the classes of GN, and in average, classes of almost $95 \%$ of the samples were distinguished correctly based the GSM-curve attributes. The majority of errors were also related to the prediction of the higher class of GN.

In the $3^{\text {rd }}$ prediction approach, the task was somewhat more complicated compared with the $2^{\text {nd }}$ approach, since the correct classes of GY and GN should be determined among the total 4 and 3 available classes, respectively. However, interestingly the performances of the prediction models improved. Table 7 represents the results of GY prediction based on GSMcurve attributes, in which the mean overall prediction accuracy was $97.41 \%$ and the $100 \%$ accuracy was frequent. The results of GN prediction were even better, as the averaged 
bioRxiv preprint doi: https://doi.org/10.1101/241786; this version posted January 13, 2019. The copyright holder for this preprint (which was not certified by peer review) is the author/funder. All rights reserved. No reuse allowed without permission.

Table 1. Contribution of the first 15 GSM curve-derived attributes (highest contributors) with grain yield, based on the normalized weights estimated by 10 weight models.

\begin{tabular}{|c|c|c|c|c|c|c|c|c|c|c|c|c|c|}
\hline \multirow[b]{3}{*}{ Rank } & \multirow[b]{3}{*}{ GSM Attributes } & \multicolumn{10}{|c|}{ Models } & \multirow[b]{3}{*}{ Count $50 \%$} & \multirow[b]{3}{*}{ Count $90 \%$} \\
\hline & & \multicolumn{10}{|c|}{ Normalized weights } & & \\
\hline & & $\begin{array}{l}\text { Weight } \\
\text { Correlation }\end{array}$ & Weight SVM & $\begin{array}{l}\text { Weight } \\
\text { Information } \\
\text { Gain Ratio }\end{array}$ & $\begin{array}{l}\text { Weight } \\
\text { Information } \\
\text { Gain }\end{array}$ & $\begin{array}{l}\text { Weight by } \\
\text { Rule }\end{array}$ & $\begin{array}{l}\text { Weight Chi } \\
\text { Squared }\end{array}$ & $\begin{array}{l}\text { Weight Gini } \\
\text { index }\end{array}$ & $\begin{array}{l}\text { Weight } \\
\text { Uncertainity }\end{array}$ & Weight Relief & $\begin{array}{l}\text { Weight } \\
\text { Deviation }\end{array}$ & & \\
\hline 1 & $\%$ Num B $B_{01} /$ unit of area & 0.22 & 1.00 & 0.88 & 0.93 & 0.57 & 0.81 & 1.00 & 1.00 & 0.70 & 1.00 & 9 & 5 \\
\hline 2 & $B_{02} / B_{04} A U C$ & 0.14 & 0.11 & 0.80 & 1.00 & 0.86 & 1.00 & 0.94 & 0.94 & 0.93 & 0.80 & 8 & 5 \\
\hline 3 & $\% \mathrm{Num} \mathrm{B}_{23} / \mathrm{CC}$ & 0.40 & 0.61 & 0.78 & 0.53 & 1.00 & 0.61 & 0.70 & 0.70 & 1.00 & 0.37 & 8 & 2 \\
\hline 4 & $\% \mathrm{Num} R_{23}$ / unit of area & 1.00 & 0.45 & 1.00 & 0.61 & 0.00 & 0.44 & 0.43 & 0.43 & 0.47 & 0.08 & 3 & 2 \\
\hline 5 & B resSlope / $R$ resSlope & 0.52 & 0.34 & 0.55 & 0.65 & 0.57 & 0.62 & 0.77 & 0.77 & 0.30 & 0.92 & 8 & 1 \\
\hline 6 & $B_{01}$ slope & 0.04 & 0.59 & 0.64 & 0.64 & 1.00 & 0.65 & 0.82 & 0.82 & 0.22 & 0.75 & 8 & 1 \\
\hline 7 & $\%$ Num $B_{0 I} / C C$ & 0.17 & 0.17 & 0.92 & 0.79 & 0.00 & 0.58 & 0.60 & 0.60 & 0.47 & 0.46 & 5 & 1 \\
\hline 8 & $\% B_{34} X$-length & 0.72 & 0.44 & 0.55 & 0.58 & 0.71 & 0.53 & 0.62 & 0.62 & 0.38 & 0.66 & 8 & 0 \\
\hline 9 & $\%$ Num $B_{23} /$ unit of area & 0.07 & 0.53 & 0.89 & 0.62 & 0.71 & 0.64 & 0.71 & 0.71 & 0.64 & 0.14 & 8 & 0 \\
\hline 10 & $B_{01}$ Lslope & 0.39 & 0.31 & 0.63 & 0.58 & 0.57 & 0.56 & 0.79 & 0.79 & 0.63 & 0.64 & 8 & 0 \\
\hline 11 & $\% \mathrm{Num} \mathrm{B}_{12} / \mathrm{CC}$ & 0.80 & 0.43 & 0.67 & 0.72 & 0.86 & 0.79 & 0.81 & 0.81 & 0.41 & 0.49 & 7 & 0 \\
\hline 12 & a coefficient of $B$ equation & 0.33 & 0.06 & 0.61 & 0.66 & 0.14 & 0.61 & 0.56 & 0.56 & 0.56 & 0.53 & 7 & 0 \\
\hline 13 & $B_{12} A U C$ & 0.51 & 0.31 & 0.72 & 0.60 & 0.43 & 0.52 & 0.55 & 0.55 & 0.65 & 0.31 & 7 & 0 \\
\hline 14 & $G B_{12} A B C$ & 0.10 & 0.32 & 0.80 & 0.77 & 0.71 & 0.75 & 0.74 & 0.74 & 0.60 & 0.44 & 7 & 0 \\
\hline 15 & Num $R_{12} /$ Num $B_{12}$ & 0.42 & 0.40 & 0.64 & 0.73 & 0.71 & 0.68 & 0.76 & 0.76 & 0.39 & 0.54 & 7 & 0 \\
\hline
\end{tabular}

The weights in each column are normalized in the range from 0 to 1 . "Count $50 \%$ " and "Count $90 \%$ " indicate the number of models by which the weight of the attribute was estimated higher than 0.5 and 0.9 , respectively (the ranks of attributes are sorted based on "count $90 \% "$ "). Num: number of pixels; B: the GSM blue curve; R: the GSM red curve; the subscripts of $01,12,23,34$, and 45 are the five GSM classes determined based on segmentation type 3; AUC: under curve area; CC: canopy coverage; Slope: mean slope of the red or blue trends in the given curve segment; \%X-length: the percentage of green range (on the horizontal axis) determined by Segmentation Type 3; Lslope: the linear slope of the red or blue trends; GB ABC: the area between green and blue curves on the GSM graph.

$B$ resSlope $/$ R resSlope $=\left(\right.$ Slope $B_{34}-1 /\left(\right.$ Slope $\left.\left.B_{12}\right)\right) /\left(\right.$ Slope $R_{34}-1 /\left(\right.$ Slope $\left.\left.R_{12}\right)\right)$

Table 2. Contribution of the first 15 GSM curve-derived attributes (highest contributors) with grains $\mathrm{m}^{-2}$, based on the normalized weights estimated by 10 weight models.

\begin{tabular}{|c|c|c|c|c|c|c|c|c|c|c|c|c|c|}
\hline \multirow[b]{3}{*}{ Rank } & \multirow[b]{3}{*}{ GSM Attributes } & \multicolumn{10}{|c|}{ Models } & \multirow[b]{3}{*}{ Count $50 \%$} & \multirow[b]{3}{*}{ Count $90 \%$} \\
\hline & & \multicolumn{10}{|c|}{ Normalized weights } & & \\
\hline & & $\begin{array}{l}\text { Weight } \\
\text { Correlation }\end{array}$ & Weight SVM & $\begin{array}{l}\text { Weight } \\
\text { Information } \\
\text { Gain Ratio }\end{array}$ & $\begin{array}{l}\text { Weight } \\
\text { Information } \\
\text { Gain }\end{array}$ & $\begin{array}{l}\text { Weight by } \\
\text { Rule }\end{array}$ & $\begin{array}{l}\text { Weight Chi } \\
\text { Squared }\end{array}$ & $\begin{array}{l}\text { Weight Gini } \\
\text { index }\end{array}$ & $\begin{array}{l}\text { Weight } \\
\text { Uncertainity }\end{array}$ & Weight Relief & $\begin{array}{l}\text { Weight } \\
\text { Deviation }\end{array}$ & & \\
\hline 1 & $B_{23}$ Slope / $B_{23}$ Lslope & 0.70 & 1.00 & 0.93 & 0.91 & 1.00 & 1.00 & 1.00 & 0.92 & 0.70 & 0.41 & 9 & 7 \\
\hline 2 & $G B_{12} A B C$ & 0.42 & 0.22 & 1.00 & 1.00 & 0.20 & 0.80 & 0.88 & 1.00 & 1.00 & 0.44 & 6 & 4 \\
\hline 3 & $B_{01}$ Lslope & 1.00 & 0.64 & 0.58 & 0.56 & 0.40 & 0.42 & 0.75 & 0.57 & 0.37 & 0.64 & 7 & 1 \\
\hline 4 & $B_{02} / B_{04} A U C$ & 0.01 & 0.36 & 0.71 & 0.91 & 0.00 & 0.70 & 0.71 & 0.78 & 0.64 & 0.80 & 7 & 1 \\
\hline 5 & $\%$ Num $B_{01}$ / unit of area & 0.27 & 0.18 & 0.76 & 0.85 & 0.00 & 0.51 & 0.87 & 0.80 & 0.04 & 1.00 & 6 & 1 \\
\hline 6 & $\% R_{12} X$-length & 0.54 & 0.25 & 0.93 & 0.68 & 0.00 & 0.38 & 0.39 & 0.81 & 0.08 & 0.11 & 4 & 1 \\
\hline 7 & B resSlope / $R$ resSlope & 0.14 & 0.22 & 0.27 & 0.33 & 0.00 & 0.20 & 0.26 & 0.30 & 0.43 & 0.92 & 1 & 1 \\
\hline 8 & $\%$ um $B_{23}$ / unit of area & 0.02 & 0.63 & 0.85 & 0.62 & 0.60 & 0.70 & 0.68 & 0.74 & 0.72 & 0.14 & 8 & 0 \\
\hline 9 & $R_{01}$ Lslope & 0.88 & 0.86 & 0.76 & 0.55 & 0.60 & 0.39 & 0.64 & 0.66 & 0.38 & 0.26 & 7 & 0 \\
\hline 10 & $\% B_{12} X$-length & 0.75 & 0.40 & 0.83 & 0.56 & 0.00 & 0.45 & 0.53 & 0.69 & 0.61 & 0.25 & 6 & 0 \\
\hline 11 & $\%$ Num $B_{12} / C C$ & 0.38 & 0.19 & 0.66 & 0.74 & 0.00 & 0.53 & 0.56 & 0.69 & 0.46 & 0.49 & 5 & 0 \\
\hline 12 & $\%$ Num $B_{23} / C C$ & 0.11 & 0.13 & 0.79 & 0.57 & 0.40 & 0.56 & 0.47 & 0.68 & 0.79 & 0.37 & 5 & 0 \\
\hline 13 & Num $B_{23} / N u m B_{05}$ & 0.11 & 0.13 & 0.79 & 0.57 & 0.40 & 0.56 & 0.47 & 0.68 & 0.79 & 0.37 & 5 & 0 \\
\hline 14 & $\%$ Num $B_{01} / C C$ & 0.25 & 0.19 & 0.74 & 0.66 & 0.00 & 0.33 & 0.51 & 0.71 & 0.23 & 0.46 & 4 & 0 \\
\hline 15 & $B_{23} A U C$ & 0.50 & 0.35 & 0.63 & 0.47 & 0.40 & 0.55 & 0.47 & 0.55 & 0.41 & 0.15 & 4 & 0 \\
\hline
\end{tabular}

The weights in each column are normalized in the range from 0 to 1 . "Count $50 \%$ " and "Count $90 \%$ " indicate the number of models by which the weight of the attribute was estimated higher than 0.5 and 0.9 , respectively (the ranks of attributes are sorted based on "count $90 \% "$ "). B and R: the GSM blue and red curves, respectively; Slope: mean slope of the red or blue curves in the given ST3 segment; Lslope: the linear slope of the red or blue trends; GB ABC: the area between green and blue curves on the GSM graph. AUC: under curve area; Num: number of pixels; the subscripts of 01 , 12 , 23, 34, and 45 are the five GSM classes determined based on segmentation type 3; \%X-length: the percentage of green range (on the horizontal axis) determined by Segmentation Type 3; CC: canopy coverage.

$B$ resSlope $/$ R resSlope $=\left(\right.$ Slope $B_{34}-1 /\left(\right.$ Slope $\left.\left.B_{12}\right)\right) /\left(\right.$ Slope $R_{34}-1 /\left(\right.$ Slope $\left.\left.R_{12}\right)\right)$ 
bioRxiv preprint doi: https://doi.org/10.1101/241786; this version posted January 13, 2019. The copyright holder for this preprint (which was not certified by peer review) is the author/funder. All rights reserved. No reuse allowed without permission.

Table 3. Model perfomances in predicting grain yield using GSM curve derived characteristics in the $1^{\text {st }}$ prediction approach.

\begin{tabular}{|c|c|c|c|c|c|c|}
\hline \multirow[b]{2}{*}{ Criteria } & \multicolumn{6}{|c|}{ Models } \\
\hline & Neural Network & Generalized Linear Model & Deep Learning & Decision Tree & Random Forest & Gradient Boosted Trees \\
\hline Root mean squared error & 1.729 & 1.32 & 1.437 & 1.606 & 1.249 & 1.309 \\
\hline Absolute error & $1.331 \pm 1.103$ & $1.064 \pm 0.781$ & $1.162 \pm 0.844$ & $1.310 \pm 0.929$ & $0.961 \pm 0.797$ & $1.023 \pm 0.817$ \\
\hline Relative error & $18.45 \% \pm 13.32 \%$ & $14.57 \% \pm 9.03 \%$ & $16.41 \% \pm 10.22 \%$ & $17.96 \% \pm 10.80 \%$ & $12.69 \% \pm 8.46 \%$ & $13.58 \% \pm 8.75 \%$ \\
\hline Relative error lenient & $16.87 \% \pm 11.81 \%$ & $13.97 \% \pm 8.73 \%$ & $15.56 \% \pm 9.72 \%$ & $17.18 \% \pm 10.33 \%$ & $12.43 \% \pm 8.52 \%$ & $13.30 \% \pm 8.78 \%$ \\
\hline Relative error strict & $23.33 \% \pm 21.88 \%$ & $17.53 \% \pm 12.80 \%$ & $20.23 \% \pm 15.96 \%$ & $22.77 \% \pm 16.42 \%$ & $15.40 \% \pm 12.44 \%$ & $16.65 \% \pm 13.02 \%$ \\
\hline Normalized absolute error & 1.426 & 1.141 & 1.246 & 1.404 & 1.03 & 1.097 \\
\hline Root relative squared error & 1.554 & 1.187 & 1.291 & 1.444 & 1.122 & 1.177 \\
\hline Squared error & $2.989 \pm 4.911$ & $1.743 \pm 2.337$ & $2.064 \pm 3.234$ & $2.580 \pm 3.277$ & $1.559 \pm 2.395$ & $1.715 \pm 2.485$ \\
\hline Correlation & 0.141 & 0.179 & 0.28 & 0.078 & 0.262 & 0.147 \\
\hline Squared correlation & 0.02 & 0.032 & 0.078 & 0.006 & 0.069 & 0.022 \\
\hline Prediction average & $6.954 \pm 1.113$ & $6.954 \pm 1.113$ & $6.954 \pm 1.113$ & $6.954 \pm 1.113$ & $6.954 \pm 1.113$ & $6.954 \pm 1.113$ \\
\hline Spearman rho & 0.065 & 0.122 & 0.321 & 0.049 & 0.227 & 0.066 \\
\hline Kendall tau & 0.034 & 0.075 & 0.244 & 0.048 & 0.143 & 0.04 \\
\hline
\end{tabular}

The highlighted cells show the best performances, based on the intended criterion.

Table 4. Model perfomances in predicting No. of grains $\mathrm{m}^{-2}$ using GSM curve derived characteristics in the $1^{\text {st }}$ prediction approach.

\begin{tabular}{|c|c|c|c|c|c|c|}
\hline \multirow[b]{2}{*}{ Criteria } & \multicolumn{6}{|c|}{ Models } \\
\hline & Neural Network & Generalized Linear Model & Deep Learning & Decision Tree & Random Forest & Gradient Boosted Trees \\
\hline Root mean squared error & 4924.122 & 3421.818 & 4558.178 & 4021.581 & 3253.888 & 3537.111 \\
\hline Absolute error & $3697.362 \pm 3252.152$ & $2462.437 \pm 2375.972$ & $3215.215 \pm 3231.003$ & $3155.200 \pm 2493.556$ & $2387.045 \pm 2211.289$ & $2622.619 \pm 2373.399$ \\
\hline Relative error & $19.76 \% \pm 15.26 \%$ & $12.38 \% \pm 10.03 \%$ & $17.54 \% \pm 18.82 \%$ & $16.73 \% \pm 11.22 \%$ & $12.16 \% \pm 9.47 \%$ & $13.44 \% \pm 10.15 \%$ \\
\hline Relative error lenient & $18.86 \% \pm 14.87 \%$ & $12.18 \% \pm 10.05 \%$ & $15.15 \% \pm 12.00 \%$ & $15.89 \% \pm 10.87 \%$ & $11.87 \% \pm 9.43 \%$ & $13.06 \% \pm 10.05 \%$ \\
\hline Relative error strict & $28.93 \% \pm 32.34 \%$ & $15.55 \% \pm 14.75 \%$ & $20.85 \% \pm 21.80 \%$ & $21.17 \% \pm 17.87 \%$ & $14.90 \% \pm 13.42 \%$ & $16.78 \% \pm 15.24 \%$ \\
\hline Normalized absolute error & 1.505 & 1.002 & 1.309 & 1.284 & 0.971 & 1.067 \\
\hline Root relative squared error & 1.68 & 1.167 & 1.555 & 1.372 & 1.11 & 1.207 \\
\hline Squared error & $24246974.798 \pm 38923911.842$ & $11708838.204 \pm 18254302.234$ & $20776987.720 \pm 48042447.729$ & $16173110.100 \pm 23742018.975$ & $10587785.196 \pm 15784865.080$ & $12511155.166 \pm 19527581.491$ \\
\hline Correlation & 0 & 0.171 & 0.007 & 0 & 0.208 & 0 \\
\hline Squared correlation & 0 & 0.029 & 0 & 0 & 0.043 & 0 \\
\hline Prediction average & $17806.327 \pm 2931.230$ & $17806.327 \pm 2931.230$ & $17806.327 \pm 2931.230$ & $17806.327 \pm 2931.230$ & $17806.327 \pm 2931.230$ & $17806.327 \pm 2931.230$ \\
\hline Spearman rho & -0.027 & 0.148 & 0.129 & -0.078 & 0.223 & -0.029 \\
\hline Kendall tau & -0.02 & 0.097 & 0.081 & -0.053 & 0.16 & -0.024 \\
\hline
\end{tabular}

The highlighted cells show the best performances, based on the intended criterion. 
Table 5. Model performances in predicting the correct classes of grain yield in the $2^{\text {nd }}$ prediction approach.

\begin{tabular}{|c|c|c|c|c|}
\hline \multirow[b]{2}{*}{ Model } & \multicolumn{4}{|c|}{ Model performance } \\
\hline & & Obs. High GY & Obs. Low GY & $\begin{array}{l}\text { Prediction } \\
\text { accuracy }(\%)\end{array}$ \\
\hline \multirow[t]{2}{*}{ Deep Learning } & Pred. High GY & 9 & 0 & \multirow{2}{*}{100} \\
\hline & Pred. Low GY & 0 & 36 & \\
\hline \multirow[t]{2}{*}{ General Linear Model } & Pred. High GY & 6 & 0 & \multirow{2}{*}{93.33} \\
\hline & Pred. Low GY & 3 & 36 & \\
\hline \multirow[t]{2}{*}{ Neural Network } & Pred. High GY & 8 & 0 & \multirow{2}{*}{97.78} \\
\hline & Pred. Low GY & 1 & 36 & \\
\hline \multirow[t]{2}{*}{ Decision Tree } & Pred. High GY & 2 & 0 & \multirow{2}{*}{84.44} \\
\hline & Pred. Low GY & 7 & 36 & \\
\hline \multirow[t]{2}{*}{ Random Forest } & Pred. High GY & 9 & 0 & \multirow{2}{*}{100} \\
\hline & Pred. Low GY & 0 & 36 & \\
\hline \multirow[t]{2}{*}{ Gradient Boosted Trees } & Pred. High GY & 9 & 0 & \multirow{2}{*}{100} \\
\hline & Pred. Low GY & 0 & 36 & \\
\hline Overall & & & & 95.93 \\
\hline
\end{tabular}

High GY and Low GY are the two classes of grain yield with the class centroids of 7.857 and 5.971 $\mathrm{Mg} / \mathrm{ha}$, respectively, resulted from K-means clustering. Threre were 9 High GY and 36 Low GY samples in the sub-dataset used for model validation. The highlighted cells show the wrong predictions. Obs. and Pred. stand for the observed and predicted classes, respectively.

Table 6. Model performances in predicting the correct classes of No. of grains $\mathrm{m}^{-2}$ in the $2^{\text {nd }}$ prediction approach.

\begin{tabular}{|c|c|c|c|c|}
\hline \multirow[b]{2}{*}{ Model } & \multicolumn{4}{|c|}{ Model performance } \\
\hline & & Obs. High GY & Obs. Low GY & $\begin{array}{l}\text { Prediction } \\
\text { accuracy }(\%)\end{array}$ \\
\hline \multirow[t]{2}{*}{ Deep Learning } & Pred. High GY & 12 & 0 & \multirow{2}{*}{100} \\
\hline & Pred. Low GY & 0 & 33 & \\
\hline \multirow[t]{2}{*}{ General Linear Model } & Pred. High GY & 7 & 0 & \multirow{2}{*}{88.89} \\
\hline & Pred. Low GY & 5 & 33 & \\
\hline \multirow[t]{2}{*}{ Neural Network } & Pred. High GY & 11 & 0 & \multirow{2}{*}{97.78} \\
\hline & Pred. Low GY & 1 & 33 & \\
\hline \multirow[t]{2}{*}{ Decision Tree } & $\overline{\text { Pred. High GY }}$ & 12 & 0 & \multirow{2}{*}{84.44} \\
\hline & Pred. Low GY & 0 & 33 & \\
\hline \multirow[t]{2}{*}{ Random Forest } & $\overline{\text { Pred. High GY }}$ & 12 & 0 & \multirow{2}{*}{100} \\
\hline & Pred. Low GY & 0 & 33 & \\
\hline \multirow[t]{2}{*}{ Gradient Boosted Trees } & Pred. High GY & 12 & 1 & \multirow{2}{*}{97.78} \\
\hline & Pred. Low GY & 0 & 32 & \\
\hline Overall & & & & 94.82 \\
\hline
\end{tabular}

High GN and Low GN are the two classes No. of grains $\mathrm{m}^{-2}$ with the class centroids of 20131.976 and 15216.531 grains $\mathrm{m}^{-2}$, respectively, resulted from K-means clustering. Threre were $12 \mathrm{High}$ GN and 33 Low GN samples in the sub-dataset used for model validation. The highlighted cells show the wrong predictions. Obs. and Pred. stand for the observed and predicted classes, respectively. 
bioRxiv preprint doi: https://doi.org/10.1101/241786; this version posted January 13, 2019. The copyright holder for this preprint (which was not certified by peer review) is the author/funder. All rights reserved. No reuse allowed without permission.

Table 7. Model performances in predicting the correct classes of grain yield in the $3^{\text {rd }}$ prediction approach.

\begin{tabular}{|c|c|c|c|c|c|c|}
\hline \multirow[b]{2}{*}{ Model } & \multicolumn{6}{|c|}{ Model performance } \\
\hline & & Obs. class 3 & Obs. class 4 & Obs. class 1 & Obs. class 2 & $\begin{array}{l}\text { Prediction } \\
\text { accuracy }(\%)\end{array}$ \\
\hline \multirow[t]{4}{*}{ Deep Learning } & Pred. class 3 & 5 & 0 & 0 & 0 & \multirow{4}{*}{100} \\
\hline & Pred. class 4 & 0 & 4 & 0 & 0 & \\
\hline & Pred. class 1 & 0 & 0 & 24 & 0 & \\
\hline & Pred. class 2 & 0 & 0 & 0 & 12 & \\
\hline \multirow[t]{4}{*}{ General Linear Model } & Pred. class 3 & 5 & 0 & 0 & 0 & \multirow{4}{*}{93.33} \\
\hline & Pred. class 4 & 0 & 1 & 0 & 0 & \\
\hline & Pred. class 1 & 0 & 1 & 24 & 0 & \\
\hline & Pred. class 2 & 0 & 2 & 0 & 12 & \\
\hline \multirow[t]{4}{*}{ ID3 } & Pred. class 3 & 5 & 0 & 0 & 0 & \multirow{4}{*}{100} \\
\hline & Pred. class 4 & 0 & 4 & 0 & 0 & \\
\hline & Pred. class 1 & 0 & 0 & 24 & 0 & \\
\hline & Pred. class 2 & 0 & 0 & 0 & 12 & \\
\hline \multirow[t]{4}{*}{ Decision Tree } & Pred. class 3 & 5 & 0 & 0 & 1 & \multirow{4}{*}{91.11} \\
\hline & Pred. class 4 & 0 & 3 & 1 & 0 & \\
\hline & Pred. class 1 & 0 & 1 & 23 & 1 & \\
\hline & Pred. class 2 & 0 & 0 & 0 & 10 & \\
\hline \multirow[t]{4}{*}{ Random Forest } & Pred. class 3 & 5 & 0 & 0 & 0 & \multirow{4}{*}{100} \\
\hline & Pred. class 4 & 0 & 4 & 0 & 0 & \\
\hline & Pred. class 1 & 0 & 0 & 24 & 0 & \\
\hline & Pred. class 2 & 0 & 0 & 0 & 12 & \\
\hline \multirow[t]{4}{*}{ Gradient Boosted Trees } & Pred. class 3 & 5 & 0 & 0 & 0 & \multirow{4}{*}{100} \\
\hline & Pred. class 4 & 0 & 4 & 0 & 0 & \\
\hline & Pred. class 1 & 0 & 0 & 24 & 0 & \\
\hline & Pred. class 2 & 0 & 0 & 0 & 12 & \\
\hline Overall & & & & & & 97.41 \\
\hline
\end{tabular}

There were 4, 5, 12, and 24 samples for the qualitative classes 4,3, 2, and 1 in the sub-dataset used for model validation; which are (in the order of class 4 to 1) the highest to lowest yielding groups, respectively (based on AHC, Agglomerative Hierarchical Clustering of values sorted in ascending order). Obs. and Pred. stand for the observed and predicted classes, respectively. 
accuracy reached $99.26 \%$ (Table 8 ). The reason for such relatively higher accuracies of the $3^{\text {rd }}$ prediction approach compared with the $2^{\text {nd }}$ one may lay in an improved model training. Indeed, it might be related to the fact that the AHC clustering method had provided a higher number of clusters with more intra-cluster homogeneity and/or inter-cluster heterogeneity, which could lead to recognizing more distinct rules and patterns in the training subset, and subsequently yielded more robust predictions. While, in the $2^{\text {nd }}$ approach, there were only two classes available; so the determined rules are expected to be more general so that they could cover a higher number and more diverse samples in each subset.

Considering that the components of the two 45-sample subsets used for model training and validation were selected by the software through randomly splitting of the 90-sample set in each running of the models (also with keeping the same class distributions in both subsets), the analyses were repeated several times (data not shown). Still, almost similar results were observed, since there were always 2 or more models with $100 \%$ accuracy (compared to the data shown in Tables 5 to 8), and the range and mean overall values were highly similar to the run whose results are reported.

Seeking the potential considerable interrelationships among the GSM-curve attributes in order to improve our understanding of GSM, pairwise correlations between the attributes were also evaluated (Table S4). As shown in Table S5, the most frequent cases of strong correlations were observed for the relationships between GSM-coverage attributes of individual $\mathrm{ST}_{3}$ segments and the respective X-length or curve-areas of that segment (e.g. between $\% B_{23} X$-length and \%Num $B_{23}$ /unit of area). This observation suggested the idea of reducing the number of GSM-curve attributes included in the prediction models through filtering out the overlapped (highly correlated) attributes, in order to reduce the computation complexity and time cost (although in the present study we had no problem with duration of estimations, it may be crucial in some situations e.g. where there is a huge number of images to be processed). Therefore, the $3^{\text {rd }}$ prediction approach was tested only using the 29 GSMcoverage attributes as input. Even though the minimum and mean overall performances declined (Tables S6 \& S7), yet the latter did not fall below 90\%, and there were some models (i.e. 2 and 3 models for GY and GN, respectively, in the reported runs) with 100\% prediction accuracy. These results seem to be acceptable where the computation complexity and time cost are crucial, and suggest that limiting the model input size to the GSM-coverage attributes may be more efficient under such situations. 
Table 8. Model performances in predicting the correct classes of No. of grain $\mathrm{m}^{-2}$ in the tested samples in the $3^{\text {rd }}$ prediction approach.

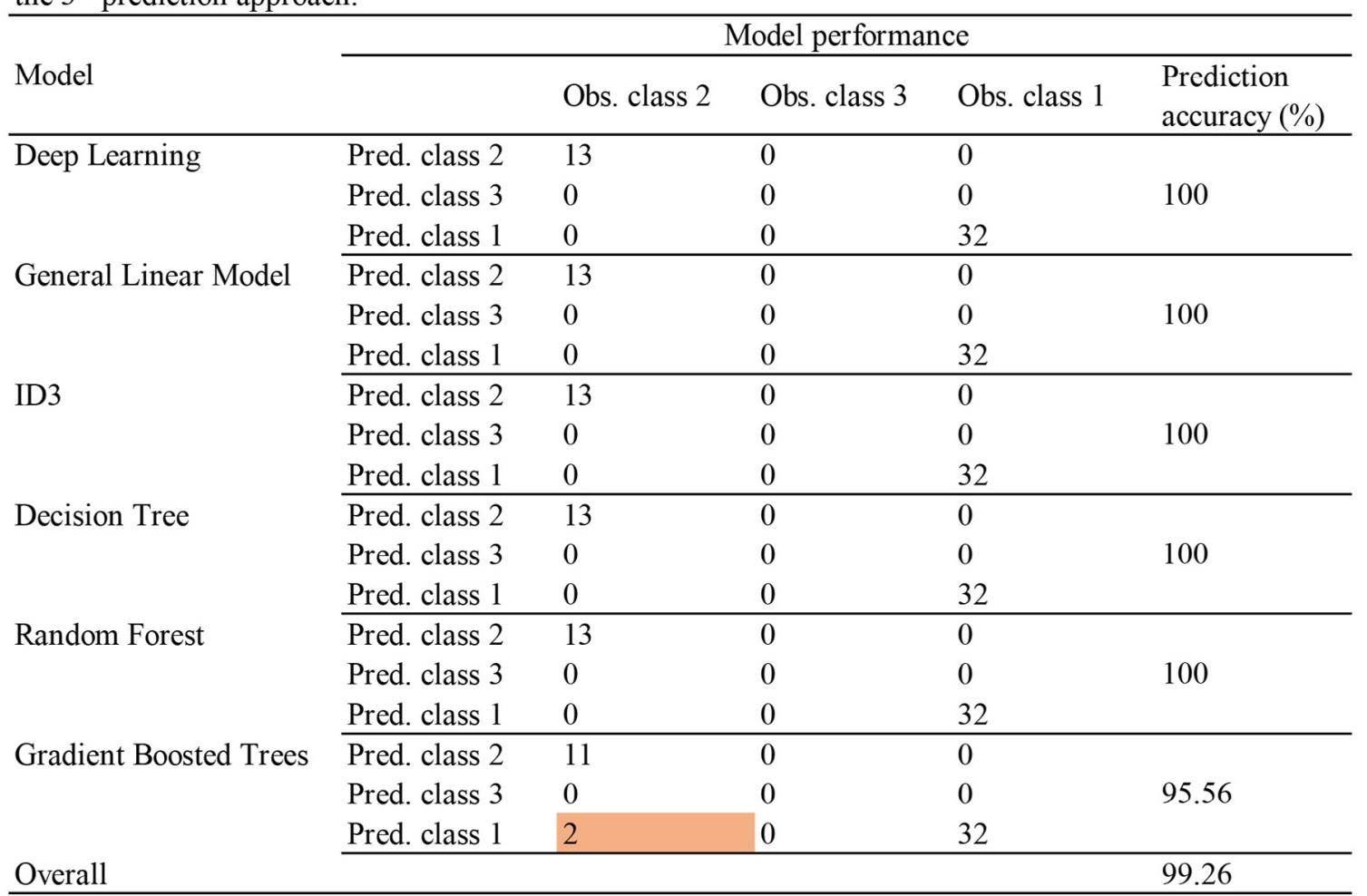

There were 13, 0, and 32 samples for the qualitative classes $4,3,2$, and 1 in the sub-dataset used for model validation; which are (in the order of class 4 to 1 ) the highest to lowest yielding groups, respectively (based on AHC, Agglomerative Hierarchical Clustering of values sorted in ascending order). It is notable that the randomly dividision of the 90 plots into two 45 -sample sets for model training and validation purposes, caused that the training set had two samples of class 3, while the validation set lacked this class. Obs. and Pred. stand for the observed and predicted classes. respectivelv. 
Besides using the statistical analyses of GSM-curve attributes, a visual comparison between GSM graphs of the canopies with the maximum and minimum GYs is provided in Fig. S7, based on which distinguishing the two groups without quantitative assessments seems difficult (if possible); although there may be some recognizable differences in the curves which needs further studies.

\subsection{GSM implications for designing canopy structure}

For assessing the potential contribution of genotype and/or number of components included in the cultivar mixtures to the GSM-curve attributes, the superior ones (i.e. "\%Num $B_{01} /$ unit of area" and " $B_{23}$ slope / $B_{23}$ Lslope" which were suggested to have maximum contributions to GY and GN, respectively) according to the 10 weight models (Tables $1 \& 2$ ) were selected and subsequently relative weights of the intended factors were estimated using weight models. As represented in Table 9 the presence of the $3^{\text {rd }}$ cultivar and also the number of components (i.e. genotypes) included in the canopy had the highest weights in determining the class of $\%$ Num Bol/unit of area. Also, where the purpose is manipulating the GSM-curve attribute $B_{23}$ slope $/ B_{23}$ Lslope, inclusion of the $4^{\text {th }}$ cultivar and the number of components may be considered as the most important contributors (Table 10). Moreover, although the single GSM-curve attributes did not show strong correlations with GY, in various analyses carried out, there were evidences for negative relationships between the attributes related to partial shadow and GY; while the attributes corresponding to the complete shadow segment seem supportive for GY (for example see Fig. 4, Fig. 5 part A; and Table S2). These examples may be evidences for the capability of GSM approach in designing a crop canopy structure. However, a combination of superior GSM-curve attributes should be considered to improve canopy structure, since as noted before, individual attributes were shown to have low correlations with GY or GN.

\subsection{Effect of Phenology on the GSM curves}

Figures 6 A, S8, and S9 show the variations of GSM curves during the $2^{\text {nd }}$ season of the field experiment, which suggest they are sensible to phenological variations and hence, capable of determining relative growing stages. Accordingly, from emergence, the overall areas between green-red and green-blue curves begin to increase up to their maximum values, with the most curvature form at the late tillering and early stem elongation phases, at the time when the most dense canopy has been formed due to intensive leaf production and low plant heights. Following stem elongation, increased canopy height lead to a returning trend, as 
bioRxiv preprint doi: https://doi.org/10.1101/241786; this version posted January 13, 2019. The copyright holder for this preprint (which was not certified by peer review) is the author/funder. All rights reserved. No reuse allowed without permission.

Table 9. Contribution of the experimental factors to "\%Num $B_{01}$ /unit of area", estimated by 10 weight models.

\begin{tabular}{|c|c|c|c|c|c|c|c|c|c|c|c|c|c|c|}
\hline \multirow[b]{3}{*}{ Rank } & \multirow[b]{3}{*}{ Experimental factors } & \multicolumn{10}{|c|}{ Models } & \multirow[b]{3}{*}{ Count $50 \%$} & \multirow{3}{*}{\multicolumn{2}{|c|}{ Count $90 \%$}} \\
\hline & & \multicolumn{10}{|c|}{ Normalized weights } & & & \\
\hline & & $\begin{array}{l}\text { Weight } \\
\text { Correlation }\end{array}$ & Weight SVM & $\begin{array}{l}\text { Weight } \\
\text { Information } \\
\text { Gain Ratio }\end{array}$ & $\begin{array}{l}\text { Weight } \\
\text { Information } \\
\text { Gain }\end{array}$ & $\begin{array}{l}\text { Weight by } \\
\text { Rule }\end{array}$ & $\begin{array}{l}\text { Weight Chi } \\
\text { Squared }\end{array}$ & $\begin{array}{l}\text { Weight Gini } \\
\text { index }\end{array}$ & $\begin{array}{l}\text { Weight } \\
\text { Uncertainity }\end{array}$ & Weight Relief & $\begin{array}{l}\text { Weight } \\
\text { Deviation }\end{array}$ & & & \\
\hline$\overline{1}$ & 3rd cultivar & 0.66 & 0.71 & 1.00 & 1.00 & 0.00 & 1.00 & 0.91 & 1.00 & 0.42 & 0.00 & & 7 & 5 \\
\hline 2 & Num of components & 0.00 & 0.00 & 0.40 & 0.96 & 1.00 & 0.98 & 1.00 & 0.69 & 0.86 & 1.00 & & 7 & 5 \\
\hline 3 & 2nd cultivar & 0.05 & 1.00 & 0.00 & 0.00 & 0.00 & 0.00 & 0.00 & 0.00 & 1.00 & 0.00 & & 2 & 2 \\
\hline 4 & Ist cultivar & 0.54 & 0.97 & 0.02 & 0.02 & 0.00 & 0.03 & 0.02 & 0.02 & 0.00 & 0.00 & & 2 & 1 \\
\hline 5 & 4th cultivar & 1.00 & 0.32 & 0.81 & 0.81 & 0.75 & 0.79 & 0.38 & 0.81 & 0.56 & 0.00 & & 7 & 1 \\
\hline
\end{tabular}

Table 10. Contribution of the experimental factors to " $B_{23}$ slope $/ R_{23} I$ slope ", estimated by 10 weight models.

\begin{tabular}{|c|c|c|c|c|c|c|c|c|c|c|c|c|c|}
\hline \multirow[b]{3}{*}{ Rank } & \multirow[b]{3}{*}{ Experimental factors } & \multicolumn{10}{|c|}{ Models } & \multirow[b]{3}{*}{ Count $50 \%$} & \multirow[b]{3}{*}{ Count $90 \%$} \\
\hline & & \multicolumn{10}{|c|}{ Normalized weights } & & \\
\hline & & $\begin{array}{l}\text { Weight } \\
\text { Correlation }\end{array}$ & Weight SVM & $\begin{array}{l}\text { Weight } \\
\text { Information } \\
\text { Gain Ratio }\end{array}$ & $\begin{array}{l}\text { Weight } \\
\text { Information } \\
\text { Gain }\end{array}$ & $\begin{array}{l}\text { Weight by } \\
\text { Rule }\end{array}$ & $\begin{array}{l}\text { Weight Chi } \\
\text { Squared }\end{array}$ & $\begin{array}{l}\text { Weight Gini } \\
\text { index }\end{array}$ & $\begin{array}{l}\text { Weight } \\
\text { Uncertainity }\end{array}$ & Weight Relief & $\begin{array}{l}\text { Weight } \\
\text { Deviation }\end{array}$ & & \\
\hline 1 & 4th cultivar & 0.72 & 1.00 & 1.00 & 0.93 & 1.00 & 1.00 & 1.00 & 1.00 & 0.00 & 0.00 & & 8 \\
\hline 2 & Num of components & 0.00 & 0.41 & 0.51 & 1.00 & 1.00 & 0.97 & 0.87 & 0.79 & 0.55 & 1.00 & & 8 \\
\hline 3 & 2nd cultivar & 0.53 & 0.00 & 0.10 & 0.09 & 1.00 & 0.10 & 0.02 & 0.10 & 1.00 & 0.00 & & 3 \\
\hline 4 & 3rd cultivar & 1.00 & 0.31 & 0.84 & 0.77 & 1.00 & 0.83 & 0.69 & 0.84 & 0.00 & 0.00 & & 7 \\
\hline 5 & Ist cultivar & 0.42 & 0.00 & 0.00 & 0.00 & 1.00 & 0.00 & 0.00 & 0.00 & 0.05 & 0.00 & & 1 \\
\hline
\end{tabular}

"B23 slope / B23 Lslope" was the most releavant GSM-derived attribute to number of grains $\mathrm{m}^{-2}$, based on the weight models (see Table 2. S). The weights in each column are normalized in the range from 0 to 1 . "Count $50 \%$ " and "Count $90 \%$ " indicate the number of models by which the weight of cultivar or number of components is estimated higher than 0.5 and 0.9 , respectively (the ranks of attributes are sorted based on "count $90 \% ")$. 
towards the end of growing season -in particular- the red trend line again approaches to the green line. These results indicated that the GSM graph may be used for monitoring and quantifying the phenological trends, considering the sensibilities kept even late in the season.

\subsection{Effects of water and cold stresses on the GSM curves}

As shown in Fig. 6 B\&C, Fig. S10, and Fig. S11, it was also observed that the GSM graph was influenced by environmental stresses i.e. comparatively abnormal diverted forms mainly made by altered (either increased or decreased) reflection of blue light under full sunlight to complete shadow conditions. Such observations may propose an acceptable diagnostic role for GSM in detecting various kinds of stresses which affect canopy optics or BDRF, especially considering the total or partial variations of the area between the blue-red curves and blue-green trends. According to the findings, it should be emphasized that $\mathrm{ST}_{3}$ is developed based on the images of wheat canopies grown under relatively optimal conditions, and thus it seems that other types of curve-segmentation may be required for stressful conditions.

Figure S12 provides additional information about different aspects of GSM, including (i) distribution of number of pixels participated in GSM green levels, and (ii) indices of dispersion, which may be used in next studies with other purposes (e.g. probably in light scattering assessments, model corrections, etc.). Moreover, only as examples of some other potential applications of GSM graphs in image-based evaluation of various types of canopies, images and graphs of several species are represented in Fig. S13 (mostly included single plants taken as individual vegetation canopies).

\section{Discussion}

In the present study, the option of utilizing a simple image mining technique for being used in HTP studies was evaluated, which is based on the canopy segmentation according to the linear gradient of green color. The archive of images used were taken from the canopies of wheat cultivar mixtures on which various assessments might be challenging both using infield measurements or through remote sensing approaches, due to including higher intraspecies heterogeneities compared with the monocultures (e.g. see Borg et al., 2018, and Reiss \& Drinkwater, 2018). However, with respect to the results provided here, still it seems that the GSM technique has acceptable performances in quantitative illustration of the reflection 

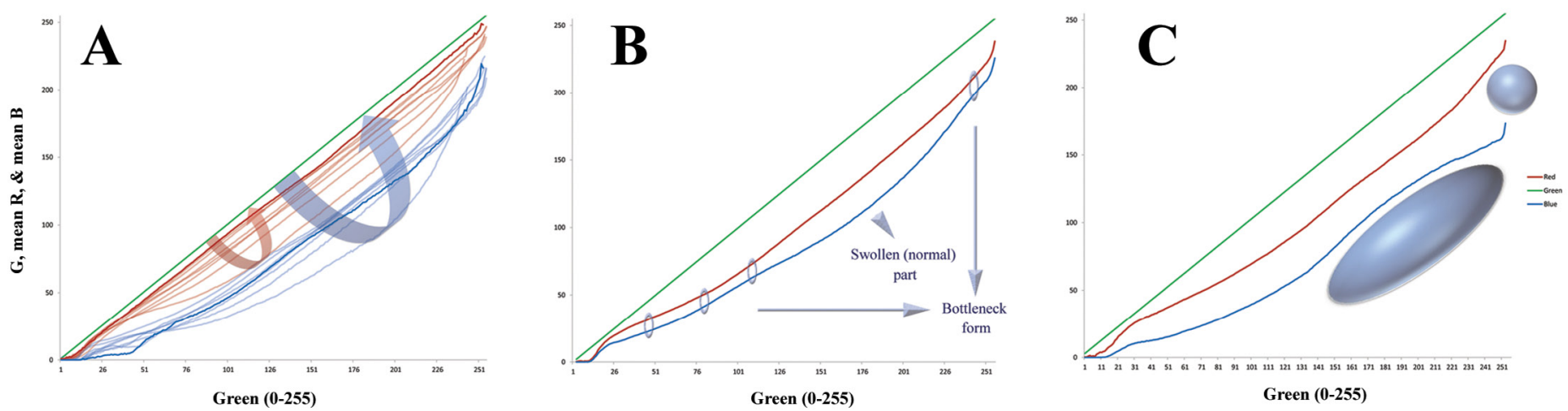

Figure 6. Evidences for the effects of phenology and environmental stresses on GSM curve. The red and blue curves are affected by (A) the canopy growth stage (phenology), (B) water and (C) cold stresses, respectively. whose diversion from the normal forms are indicated for the latter two as a bottleneck form or by ellipsoids. For more details, see Figs. S8 to S11. 
status above the canopy in a simple form, discrimination of canopy green surfaces according to their exposure to sunlight (e.g. distinguishing between partial- and complete shadows), and eventually predicting the classes of grain yield and number of grains $\mathrm{m}^{-2}$ with high accuracies. Moreover, there were evidences for the GSM sensibility to the environmental stresses, based on which it may be improved for stress studies.

The GSM graph has an intrinsic simplicity arises from its simple structure, non-complex computations (i.e. based on a routine pixel classification, and basic statistics), and straightforward interpretation (according to the comparative variations in curve slopes, which may show the relative variations in canopy color -or visible reflection- under changing illumination). Despite such simplicities, GSM graph extracts and represents an orderly pattern from a naturally complex and disordered phenomenon i.e. the extremely sophisticated light scattering above the canopy. Based on this fact, GSM seems to go beyond a technique, and may be readily used as an identification concept for vegetation canopies. In other words, in an ideal scope and after justified by adequate studies, a vegetation canopy may be quantitatively defined and distinguished from others, using the overall form of its GSM graph or equations of the curves. As described before, GSM graph includes a comprehensive collection of sensed reflections from surfaces with different arrangements and positions in the canopy, i.e. located under various illuminations and exposures to sunlight. That is to say, assuming the whole canopy as a green flat homogenous surface unit (e.g. as a conceptual pixel), GSM may be comparable with the integrative and comprehensive evaluation of the visible spectral behavior of this unit from a fixed point of view, when it is experiencing a complete set of changing illuminations (full sunlight to full- or deep-shadow) and angle. However, it should be noted that sometimes (depended on the study purposes), canopy cannot be assumed as a single unit with homogenous reflectance behavior; e.g. due to the heterogeneities in the pattern of leaf senescence, and/or nitrogen distribution across the canopy height (or between the sun-exposed vs. shaded surfaces; see Dreccer et al., 2000; Bertheloot et al., 2008; Hikosaka et al., 2016; Kitao et al., 2018). Under such conditions, the aim of the study determines if these variations should be either neglected, or taken into account. Anyway, the GSM graph indicates the variations in the relative reflections of the red and blue bands, regardless the biological reasons. Overall, comparing the various feasibilities in implementation and utilization of GSM with the its various applications and outputs, may suggest it as an efficient image-based HTP platform. 
In addition to the potential applications described for the overall form of GSM graph, a further analysis on the curves lead to a curve-based segmentation approach ( $\left.\mathrm{ST}_{3}\right)$, based on which the canopy was segmented into 5 classes from full-sunlight to deep-shadow, with acceptable accuracies (see Fig. S2). The criterion used for $\mathrm{ST}_{3}$ segmentation was continuous variations in local slopes of red or blue curves, relative to the fixed slope of the green trend (i.e. equals 1). Accordingly, individual shading patterns and series of thresholding values were recognized for each of the red or blue curves; which implies that $\mathrm{ST}_{3}$ distinguishes between the red-color-based and blue-color-based shading, while their results are highly overlapped in most cases. However, it should be kept in mind that the equations and/or equation fitness of GSM curves may be changed under stressful and abnormal conditions. So the $\mathrm{ST}_{3}$ may also be required to be carried out in a different way; e.g. through segmenting the GSM curves into only two segments based upon having slopes either more or less than 1, or alternatively, using slope-independent curve attributes such as curve quantiles described (for example, see attributes " $R Q_{2}$ " or "B $Q_{3}$ " in Table S1 and Fig. S3 A). Indeed, the GSM approach seems to be comprehensive and also flexible enough to provide the opportunity for calculating such diversions compared with the typical form.

One of the most important aspects of GSM method evaluated was its function in prediction of GY and GN. Yield prediction is considered as the main purpose of many remote sensing and digital imagery studies (Alganci et al., 2014; Wang et al., 2014; Pantazi et al., 2016; Chen and Jing, 2017; Chlingaryan et al., 2018; Donohue et al., 2018; Hassan et al., 2018; Lai et al., 2018; Schut et al., 2018; Walter et al., 2018; Reza et al., 2019), due to its priority in crop sciences and practices. Also in the present study, it was expected that GY and its important contributor, GN, would be desirable choices for examining the GSM practical application and assessing the validation of its function. Among grain yield components, number of spikes $\mathrm{m}^{-2}$ had been almost determined before the imaging date, and mean grain weight (MGW) would also be determined mainly in the future phases (although the potential of MGW may be determined by events up to about a week before or after anthesis, it is mainly associated with the grain filling period; Fischer, 2011). Therefore, the number of grains $\mathrm{m}^{-2}$ was selected and evaluated as the most relevant grain yield component to the crop phase in which the images were taken (for instance, consider the relative contribution of preanthesis days to the grain yield components formation reviewed by Fischer, 2011; and also Slafer et al., 2014). It is notable that in the present study, the recorded flowering date for the four early- to middle-ripening $\left(1^{\text {st }}\right.$ to $\left.4^{\text {th }}\right)$ cultivars were $2,4,8$, and 11 days after the imaging 
date, respectively. As shown earlier, none of the individual GSM-curve attributes had strong correlation (R values higher than about 0.3 ) with GY and GN. Therefore, their combination was used as the input of data mining models for predictions. Still, where the quantitative values were used (in the $1^{\text {st }}$ prediction approach), the prediction performances were not satisfactory, and the model outputs values were concentrated around the mean values of observations, with least accuracies in predicting extremes. Probably these low accuracies for predictions resulted from using quantitative values may be due to: (i) the theoretical weights of the GSM-curve attributes in GY and GN quantitative prediction, or in other words, the natural contribution of attributes to forecasting GY or GN quantities may be almost limited to the reported accuracies. (ii) It may be associated with the size of models training set, which was composed of 45 images -or experimental plots-; and thus, it may be possible to achieve acceptable accuracy using higher number of images of wheat canopies. (iii) The quantitative predictions may have been affected by inclusion of the deficit-irrigated samples in the evaluations. Therefore, it seems that further studies are required for understanding the restrictions and potential options for improving the accuracy of quantitative predictions.

Despite the $1^{\text {st }}$ prediction approach, the datamining models indicated maximum possible performances in predicting the qualitative classes of GY and GN predictions based on GSMcurve attributes (in the $2^{\text {nd }}$ and $3^{\text {rd }}$ approaches); as always (in each run) there were at least one or two models those predicted the classes of all plots correctly (with 100\% accuracy). It may provide the option of predicting the class of GY only by analyzing the images of canopies taken at booting phase, particularly where considerable number of genotypes or treatments should be screened for high GY (or GN), e.g. in the local to international breeding programs (e.g. see Lopes et al., 2014; Mondal et al., 2016; Crespo-Herrera et al., 2017; MontesinosLópez et al., 2017; and Lopes et al., 2018). Furthermore, when the number of total GSMcurve attributes were limited to the GSM-coverage series (more than $80 \%$ reduction in number of attributes), the model performances still remained high; which implies an increase in the relative computation efficiency of the GSM-based prediction approach. Here, it should be emphasized that although there may be a need for further evaluation of biophysiological background of GSM-curve attributes in some surveys, they can be used without understanding the corresponding biological mechanisms, when they are utilized as validated HTP indices. For example, Cabera-Bosquet et al. (2012) believed: "Both GS (genomic selection) and high-throughput phenotyping have in common their empirical approach enabling breeders to use genome profile or phenotype without understanding the underlying 
biology". Moreover, the simplicity of interpreting the biological mechanisms of GSM-curve attributes might be different, and depended on the type of attributes (e.g. see AUCs vs. coefficients of exponential red or blue curves).

In a brief assessment, effects of presence of each genotype and number of components in the mixtures on the GSM-curve attributes with highest contribution to GY and GN were evaluated. The results indicated the potential capability of GSM method as an advantageous HTP tool for monitoring the processes of designing or manipulating canopy structure without in-field tedious measurements.

Besides, a potential diagnostic role may be suggested for GSM, based on its sensibility to crop phenology and stressful conditions. At the current level, GSM may be used for monitoring the variations in the pattern of visible light scattering above the crop canopies at a given phenological stage, or being under stress; however, in the cases where a curve-based segmentation method (similar to $\mathrm{ST}_{3}$ ) is required for further analysis, it might be required to utilize other appropriate (and probably exclusive) techniques.

Researches with the aim of evaluating the structure and optics of vegetation canopies, have utilized a relatively broad spectrum of physical bases, mathematical models, and practically, novel photometric instruments (e.g. Verhoef, 1984; Welles and Cohen, 1996; Gower et al., 1999; Jonckheere I. et al., 2004; Omasa et al., 2006; Jacquemoud et al., 2009; Zhao et al., 2011; Glatthorn and Beckschäfer, 2014; Bauer et al., 2016; Yao et al., 2016). Despite the diversity and evolution of the methodologies, the two early introduced concepts of leaf area index -LAI- (Watson, 1947) and the exponential trend of light attenuation (i.e. based upon Beer-Lambert law; Monsi and Saeki, 2005), solely or in combination, have been the cornerstones of almost every conventional analyses in this context. Accordingly, leaves (with their individual characteristics e.g. type, angle, configuration) act as the light attenuating materials, and LAI is comparable with the material concentration. Conventional measurement approaches include estimating the conceptual index of LAI either by destructive sampling or indirect methods (Weiss et al., 2004; Behera et al., 2010; Liu et al., 2010; Viña et al., 2011; Mu et al., 2017), and/or modeling the light extinction trend based on recorded light intensities by in situ sensors (Jonckheere et al., 2004; Munier-Jolain et al., 2013; Xue et al., 2015; Perot et al., 2017). In these methods, even results of remote sensing analyses are calibrated based on LAI or in-field sensor outputs, which may be a laborious practice and usually requires utilization of complex models. 
Although here the focus was basically on evaluating the GSM method as a HTP platform, it seems to have the potential to contribute to canopy optics and biophysical assessments. Indeed, GSM is expected to have strong contribution with canopy BDRF and may be used to measure canopy radiation field and quantifying the scattering process inside vegetation canopies, which also requires to be compared with the results of valid physical methodologies in next studies based on the in-situ measurements and/or combining the GSM-based findings with developed radiative transfer models (e.g. see Vilfan et al., 2016; Yang et al., 2017; and Li et al., 2018). It is notable that here, no in-field measurement was carried out for analyzing the canopy architecture properties (e.g. measuring PAR, or leaf area for LAI -leaf area indexestimations), since the idea of the study was formed after field harvest, and hence, the focus was basically put on evaluating the option of using GSM method as a HTP platform for GY analyses (whose data was available for performance validation). While in absence of direct and conventional measurements of the canopy structure properties (or optics) no argumentation or claim has been made about these aspects in the physical level, yet, based on the results of image segmentation $\left(\mathrm{ST}_{3}\right)$, GSM seems to indirectly provide valuable information about the canopy structure. As indicated before, the GSM $\mathrm{ST}_{3}$ is capable to segment the canopy image into several parts based on the interaction between exposure to sunlight and spectral behavior of green surfaces; which is obviously supported by a quantitative and mathematical basis and yielded valid and acceptable results according to visual comparisons (Fig. S2). It may be implicated that this type of segmentation and the respective GSM attributes evaluated, are based on the shading-pattern inside the canopy, and consequently are expected to be highly contributed with canopy architecture, including leaf (green surface) arrangement, leaf angles, canopy openness, canopy height, etc. Considering the accuracies reported for prediction of GY and GN classes using $\mathrm{ST}_{3}$-derived attributes, which is an evidence for validation of GSM, a question may arise concerning utilizing the GSM and other remote sensing techniques as parallel approaches to the conventional methodologies in canopy studies (particularly in canopy structure evaluations): can they provide the opportunity for evaluating the canopy structure (or architecture) using autonomous analyzing platforms independent from the conventional laborious and sophisticated methodologies? Although finding the answer requires more evaluations, at least in the case of the present study, it seems that there are evidences for the possibility of achieving valid and (based on the purposes) adequate information about the canopy structure without necessarily utilizing or referring to the conventional procedures. Of course, novel approaches may also need new definitions, at least at the technical level. For instance, in 
some cases canopy depth (and/or layer) may be defined as an optical term (e.g. based on $\mathrm{GSM} \mathrm{ST}_{3}$ classes, or the green level itself) instead of being a spatial concept; because green surfaces may be completely subjected to either sunlight or shadow at any height in the canopy, though, the frequency (or probability) of exposures may be predictable. Accordingly, depending on purposes, (once segmented from background i.e. $\mathrm{ST}_{1}$ ) even a rolled leaf that casts a shadow on itself can be considered as an individual canopy with its own GSM graph, which also may be comparable with a wheat canopy or even with a forest in a satellite image (although such given canopies may even have similar GSM graphs -or curve equations-, they are different in tempo-spatial scales -and in the purposes- for which the identical graphs are made, and thus in the interpretation bases).

In summary, the GSM technique which is based on a simple image segmentation, may be extended into a multipurpose platform for HTP and canopy studies. It seems to be capable to contribute to the evaluations of canopy structure and optics with various degrees of dependence to the conventional methodologies and biophysical levels, e.g. in potential combination with radiative transfer models, or even as an independent approach for monitoring and assessment of image-derived properties which are indirectly associated with canopy structure (and/or architecture). Yet, further studies are needed to improve our understanding of GSM and its various applications.

\section{Conclusion}

In the present study, the option of developing an image mining platform based on a simple image segmentation technique (GSM), with the aim of using in canopy studies and highthroughput-phenotyping was evaluated. It was shown that GSM may be a novel multipurpose approach with the potential applications in:

- Quantitative identification of crop canopies in a simple form (e.g. as one or two equations, or even by the GSM graph, itself);

- Recognition and classification of shading pattern inside the canopy;

- Prediction of the qualitative classes of GY and GN with high accuracies, using the GSM-curve derived attributes as HTP vegetation indices, e.g. with application in grain yield screening programs;

- Monitoring and assessing the process of designing or manipulating canopy structure;

- Assessing the effect of canopy phenology and environmental stresses on canopy visible reflectance; and 
- Studying canopy optics, e.g. for evaluation of the radiation field above the canopy, BDRF, and pattern of visible light scattering inside the canopy (only after validation by appropriate biophysical measurements or models).

It seems that the findings reported here, including the novel technique and concepts as well as the suggested applications, may improve our knowledge of crop canopy status and also provide new vegetation indices for crop phenotyping.

\section{Acknowledgements}

The authors wish to thank Shiraz University for providing field experiment facilities. Mr. Saeid Jafarizadeh is gratefully acknowledged for his contribution in writing MATLAB codes.

\section{References}

Alganci, U., Ozdogan, M., Sertel, E., \& Ormeci, C. (2014). Estimating maize and cotton yield in southeastern Turkey with integrated use of satellite images, meteorological data and digital photographs. Field Crops Research, 157, 8-19

Araus, J.L., \& Cairns, J.E. (2014). Field high-throughput phenotyping: the new crop breeding frontier. Trends in Plant Science, 19, 52-61

Araus, J.L., Kefauver, S.C., Zaman-Allah, M., Olsen, M.S., \& Cairns, J.E. (2018). Translating High-Throughput Phenotyping into Genetic Gain. Trends in Plant Science, 23, 451-466

Bauer, J., Siegmann, B., Jarmer, T., \& Aschenbruck, N. (2016). On the potential of Wireless Sensor Networks for the in-situ assessment of crop leaf area index. Computers and Electronics in Agriculture, 128, 149-159

Behera, S.K., Srivastava, P., Pathre, U.V., \& Tuli, R. (2010). An indirect method of estimating leaf area index in Jatropha curcas L. using LAI-2000 Plant Canopy Analyzer. Agricultural and Forest Meteorology, 150, 307-311

Bertheloot, J., Martre, P., \& Andrieu, B. (2008). Dynamics of Light and Nitrogen Distribution during Grain Filling within Wheat Canopy. Plant Physiology, 148, $1707-$ 1720

Borg, J., Kiær, L.P., Lecarpentier, C., Goldringer, I., Gauffreteau, A., Saint-Jean, S., Barot, S., \& Enjalbert, J. (2018). Unfolding the potential of wheat cultivar mixtures: A meta- 
analysis perspective and identification of knowledge gaps. Field Crops Research, 221, 298-313

Cabrera-Bosquet, L., Crossa, J., von Zitzewitz, J., Serret, M.D., \& Luis Araus, J. (2012). High-throughput Phenotyping and Genomic Selection: The Frontiers of Crop Breeding ConvergeF. Journal of Integrative Plant Biology, 54, 312-320

Casadesús, J., Kaya, Y., Bort, J., Nachit, M.M., Araus, J.L., Amor, S., Ferrazzano, G., Maalouf, F., Maccaferri, M., Martos, V., Ouabbou, H., \& Villegas, D. (2007). Using vegetation indices derived from conventional digital cameras as selection criteria for wheat breeding in water-limited environments. Annals of Applied Biology, 150, $227-$ 236

Chen, D., Neumann, K., Friedel, S., Kilian, B., Chen, M., Altmann, T., \& Klukas, C. (2014). Dissecting the Phenotypic Components of Crop Plant Growth and Drought Responses Based on High-Throughput Image Analysis. The Plant Cell, 26, 4636-4655

Chen, P., \& Jing, Q. (2017). A comparison of two adaptive multivariate analysis methods (PLSR and ANN) for winter wheat yield forecasting using Landsat-8 OLI images. Advances in Space Research, 59, 987-995

Chlingaryan, A., Sukkarieh, S., \& Whelan, B. (2018). Machine learning approaches for crop yield prediction and nitrogen status estimation in precision agriculture: A review. Computers and Electronics in Agriculture, 151, 61-69

Crain, J., Mondal, S., Rutkoski, J., Singh, R.P., \& Poland, J. (2018). Combining HighThroughput Phenotyping and Genomic Information to Increase Prediction and Selection Accuracy in Wheat Breeding. The Plant Genome, 11

Crespo-Herrera, L.A., Crossa, J., Huerta-Espino, J., Autrique, E., Mondal, S., Velu, G., Vargas, M., Braun, H.J., \& Singh, R.P. (2017). Genetic Yield Gains In CIMMYT’s International Elite Spring Wheat Yield Trials By Modeling The Genotype $\times$ Environment Interaction. Crop Science, 57, 789-801

Donohue, R.J., Lawes, R.A., Mata, G., Gobbett, D., \& Ouzman, J. (2018). Towards a national, remote-sensing-based model for predicting field-scale crop yield. Field Crops Research, 227, 79-90

Dreccer, M.F., van Oijen, M., Schapendonk, A.H.C.M., Pot, C.S., \& Rabbinge, R. (2000). Dynamics of Vertical Leaf Nitrogen Distribution in a Vegetative Wheat Canopy. Impact on Canopy Photosynthesis. Annals of Botany, 86, 821-831

Easlon, H.M., \& Bloom, A.J. (2014). Easy Leaf Area: Automated digital image analysis for rapid and accurate measurement of leaf area. Applications in Plant Sciences, 2, 
apps. 1400033

Fischer, R.A. (2011). Wheat physiology: a review of recent developments. Crop and Pasture Science, 62, 95-114

Glatthorn, J., \& Beckschäfer, P. (2014). Standardizing the Protocol for Hemispherical Photographs: Accuracy Assessment of Binarization Algorithms. PLOS ONE, 9, e111924

Gower, S.T., Kucharik, C.J., \& Norman, J.M. (1999). Direct and Indirect Estimation of Leaf Area Index, fAPAR, and Net Primary Production of Terrestrial Ecosystems. Remote Sensing of Environment, 70, 29-51

Haghshenas, A., \& Emam, Y. (2019). Image-based tracking of ripening in wheat cultivar mixtures: A quantifying approach parallel to the conventional phenology. Computers and Electronics in Agriculture, 156, 318-333

Hassan, M.A., Yang, M., Rasheed, A., Yang, G., Reynolds, M., Xia, X., Xiao, Y., \& He, Z. (2018). A rapid monitoring of NDVI across the wheat growth cycle for grain yield prediction using a multi-spectral UAV platform. Plant Science

Hikosaka, K., Anten, N.P.R., Borjigidai, A., Kamiyama, C., Sakai, H., Hasegawa, T., Oikawa, S., Iio, A., Watanabe, M., Koike, T., Nishina, K., \& Ito, A. (2016). A metaanalysis of leaf nitrogen distribution within plant canopies. Annals of Botany, 118, 239-247

Hunt Jr, E.R., Doraiswamy, P.C., McMurtrey, J.E., Daughtry, C.S.T., Perry, E.M., \& Akhmedov, B. (2013). A visible band index for remote sensing leaf chlorophyll content at the canopy scale. International Journal of Applied Earth Observation and Geoinformation, 21, 103-112

Jacquemoud, S., Verhoef, W., Baret, F., Bacour, C., Zarco-Tejada, P.J., Asner, G.P., François, C., \& Ustin, S.L. (2009). PROSPECT + SAIL models: A review of use for vegetation characterization. Remote Sensing of Environment, 113, Supplement 1, S56S66

Jonckheere, I., Fleck, S., Nackaerts, K., Muys, B., Coppin, P., Weiss, M., \& Baret, F. (2004). Review of methods for in situ leaf area index determination: Part I. Theories, sensors and hemispherical photography. Agricultural and Forest Meteorology, 121, 19-35

Jonckheere, I., Fleck, S., Nackaerts, K., Muys, B., Coppin, P., Weiss, M., \& Baret, F. (2004). Review of methods for in situ leaf area index determination: Part I. Theories, sensors and hemispherical photography. Agricultural and Forest Meteorology, 121, 19-35

Kitao, M., Kitaoka, S., Harayama, H., Tobita, H., Agathokleous, E., \& Utsugi, H. (2018). 
Canopy nitrogen distribution is optimized to prevent photoinhibition throughout the canopy during sun flecks. Scientific Reports, 8, 503

Lai, Y.R., Pringle, M.J., Kopittke, P.M., Menzies, N.W., Orton, T.G., \& Dang, Y.P. (2018). An empirical model for prediction of wheat yield, using time-integrated Landsat NDVI. International Journal of Applied Earth Observation and Geoinformation, 72, 99-108

Lee, K.-J., \& Lee, B.-W. (2013). Estimation of rice growth and nitrogen nutrition status using color digital camera image analysis. European Journal of Agronomy, 48, 57-65

Li, W., Guo, Q., Tao, S., \& Su, Y. (2018). VBRT: A novel voxel-based radiative transfer model for heterogeneous three-dimensional forest scenes. Remote Sensing of Environment, 206, 318-335

Li, Y., Chen, D., Walker, C.N., \& Angus, J.F. (2010). Estimating the nitrogen status of crops using a digital camera. Field Crops Research, 118, 221-227

Liu, J., \& Pattey, E. (2010). Retrieval of leaf area index from top-of-canopy digital photography over agricultural crops. Agricultural and Forest Meteorology, 150, 1485 1490

Lopes, M.S., Rebetzke, G.J., \& Reynolds, M. (2014). Integration of phenotyping and genetic platforms for a better understanding of wheat performance under drought. J Exp Bot, $65,6167-6177$

Lopes, M.S., Royo, C., Alvaro, F., Sanchez-Garcia, M., Ozer, E., Ozdemir, F., Karaman, M., Roustaii, M., Jalal-Kamali, M.R., \& Pequeno, D. (2018). Optimizing Winter Wheat Resilience to Climate Change in Rain Fed Crop Systems of Turkey and Iran. Frontiers in Plant Science, 9

McCree, K.J. (1972). Test of current definitions of photosynthetically active radiation against leaf photosynthesis data. Agricultural Meteorology, 10, 443-453

Mondal, S., Singh, R.P., Mason, E.R., Huerta-Espino, J., Autrique, E., \& Joshi, A.K. (2016). Grain yield, adaptation and progress in breeding for early-maturing and heat-tolerant wheat lines in South Asia. Field Crops Research, 192, 78-85

Monsi, M., \& Saeki, T. (2005). On the Factor Light in Plant Communities and its Importance for Matter Production. Annals of Botany, 95, 549-567

Montesinos-López, O.A., Montesinos-López, A., Crossa, J., de los Campos, G., Alvarado, G., Suchismita, M., Rutkoski, J., González-Pérez, L., \& Burgueño, J. (2017). Predicting grain yield using canopy hyperspectral reflectance in wheat breeding data. Plant Methods, 13, 4 
Mu, X., Hu, R., Zeng, Y., McVicar, T.R., Ren, H., Song, W., Wang, Y., Casa, R., Qi, J., Xie, D., \& Yan, G. (2017). Estimating structural parameters of agricultural crops from ground-based multi-angular digital images with a fractional model of sun and shade components. Agricultural and Forest Meteorology, 246, 162-177

Munier-Jolain, N.M., Guyot, S.H.M., \& Colbach, N. (2013). A 3D model for light interception in heterogeneous crop:weed canopies: Model structure and evaluation. Ecological Modelling, 250, 101-110

Omasa, K., Hosoi, F., \& Konishi, A. (2006). 3D lidar imaging for detecting and understanding plant responses and canopy structure. J Exp Bot, 58, 881-898

Pantazi, X.E., Moshou, D., Alexandridis, T., Whetton, R.L., \& Mouazen, A.M. (2016). Wheat yield prediction using machine learning and advanced sensing techniques. Computers and Electronics in Agriculture, 121, 57-65

Perot, T., Mårell, A., Korboulewsky, N., Seigner, V., \& Balandier, P. (2017). Modeling and predicting solar radiation transmittance in mixed forests at a within-stand scale from tree species basal area. Forest Ecology and Management, 390, 127-136

Reiss, E.R., \& Drinkwater, L.E. (2018). Cultivar mixtures: a meta-analysis of the effect of intraspecific diversity on crop yield. Ecological Applications, 28, 62-77

Reza, M.N., Na, I.S., Baek, S.W., \& Lee, K.-H. (2019). Rice yield estimation based on Kmeans clustering with graph-cut segmentation using low-altitude UAV images. Biosystems Engineering, 177, 109-121

Sakamoto, T., Gitelson, A.A., Nguy-Robertson, A.L., Arkebauer, T.J., Wardlow, B.D., Suyker, A.E., Verma, S.B., \& Shibayama, M. (2012). An alternative method using digital cameras for continuous monitoring of crop status. Agricultural and Forest Meteorology, 154-155, 113-126

Schut, A.G.T., Traore, P.C.S., Blaes, X., \& de By, R.A. (2018). Assessing yield and fertilizer response in heterogeneous smallholder fields with UAVs and satellites. Field Crops Research, 221, 98-107

Slafer, G.A., Savin, R., \& Sadras, V.O. (2014). Coarse and fine regulation of wheat yield components in response to genotype and environment. Field Crops Research, 157, $71-83$

Sun, J., Nishio, J.N., \& Vogelmann, T.C. (1998). Green Light Drives CO2 Fixation Deep within Leaves1. Plant and Cell Physiology, 39, 1020-1026

Tanger, P., Klassen, S., Mojica, J.P., Lovell, J.T., Moyers, B.T., Baraoidan, M., Naredo, M.E.B., McNally, K.L., Poland, J., Bush, D.R., Leung, H., Leach, J.E., \& McKay, 
J.K. (2017). Field-based high throughput phenotyping rapidly identifies genomic regions controlling yield components in rice. Scientific Reports, 7, 42839

Terashima, I., Fujita, T., Inoue, T., Chow, W.S., \& Oguchi, R. (2009). Green Light Drives Leaf Photosynthesis More Efficiently than Red Light in Strong White Light: Revisiting the Enigmatic Question of Why Leaves are Green. Plant and Cell Physiology, 50, 684-697

Verhoef, W. (1984). Light scattering by leaf layers with application to canopy reflectance modeling: The SAIL model. Remote Sensing of Environment, 16, 125-141

Vilfan, N., van der Tol, C., Muller, O., Rascher, U., \& Verhoef, W. (2016). Fluspect-B: A model for leaf fluorescence, reflectance and transmittance spectra. Remote Sensing of Environment, 186, 596-615

Viña, A., Gitelson, A.A., Nguy-Robertson, A.L., \& Peng, Y. (2011). Comparison of different vegetation indices for the remote assessment of green leaf area index of crops. Remote Sensing of Environment, 115, 3468-3478

Walter, J., Edwards, J., McDonald, G., \& Kuchel, H. (2018). Photogrammetry for the estimation of wheat biomass and harvest index. Field Crops Research, 216, 165-174

Wang, L., Tian, Y., Yao, X., Zhu, Y., \& Cao, W. (2014). Predicting grain yield and protein content in wheat by fusing multi-sensor and multi-temporal remote-sensing images. Field Crops Research, 164, 178-188

Wang, Y., Shi, P., Zhang, G., Ran, J., Shi, W., \& Wang, D. (2016). A critical nitrogen dilution curve for japonica rice based on canopy images. Field Crops Research, 198, 93-100

Wang, Y., Wang, D., Zhang, G., \& Wang, J. (2013). Estimating nitrogen status of rice using the image segmentation of G-R thresholding method. Field Crops Research, 149, 3339

Watson, D.J. (1947). Comparative Physiological Studies on the Growth of Field Crops: I. Variation in Net Assimilation Rate and Leaf Area between Species and Varieties, and within and between Years. Annals of Botany, 11, 41-76

Weiss, M., Baret, F., Smith, G.J., Jonckheere, I., \& Coppin, P. (2004). Review of methods for in situ leaf area index (LAI) determination: Part II. Estimation of LAI, errors and sampling. Agricultural and Forest Meteorology, 121, 37-53

Welles, J.M., \& Cohen, S. (1996). Canopy structure measurement by gap fraction analysis using commercial instrumentation. J Exp Bot, 47, 1335-1342

Xue, H., Han, Y., Li, Y., Wang, G., Feng, L., Fan, Z., Du, W., Beifang, Y., Cao, C., \& Mao, 
S. (2015). Spatial distribution of light interception by different plant population densities and its relationship with yield. Field Crops Research, 184, 17-27

Yang, P., Verhoef, W., \& van der Tol, C. (2017). The mSCOPE model: A simple adaptation to the SCOPE model to describe reflectance, fluorescence and photosynthesis of vertically heterogeneous canopies. Remote Sensing of Environment, 201, 1-11

Yao, H., Zhang, Y., Yi, X., Zhang, X., \& Zhang, W. (2016). Cotton responds to different plant population densities by adjusting specific leaf area to optimize canopy photosynthetic use efficiency of light and nitrogen. Field Crops Research, 188, 10-16

Zhang, X., Huang, C., Wu, D., Qiao, F., Li, W., Duan, L., Wang, K., Xiao, Y., Chen, G., Liu, Q., Xiong, L., Yang, W., \& Yan, J. (2017). High-Throughput Phenotyping and QTL Mapping Reveals the Genetic Architecture of Maize Plant Growth. Plant Physiology, $173,1554-1564$

Zhao, F., Yang, X., Schull, M.A., Román-Colón, M.O., Yao, T., Wang, Z., Zhang, Q., Jupp, D.L.B., Lovell, J.L., Culvenor, D.S., Newnham, G.J., Richardson, A.D., Ni-Meister, W., Schaaf, C.L., Woodcock, C.E., \& Strahler, A.H. (2011). Measuring effective leaf area index, foliage profile, and stand height in New England forest stands using a fullwaveform ground-based lidar. Remote Sensing of Environment, 115, 2954-2964

Zou, X., Mõttus, M., Tammeorg, P., Torres, C.L., Takala, T., Pisek, J., Mäkelä, P., Stoddard, F.L., \& Pellikka, P. (2014). Photographic measurement of leaf angles in field crops. Agricultural and Forest Meteorology, 184, 137-146 
A

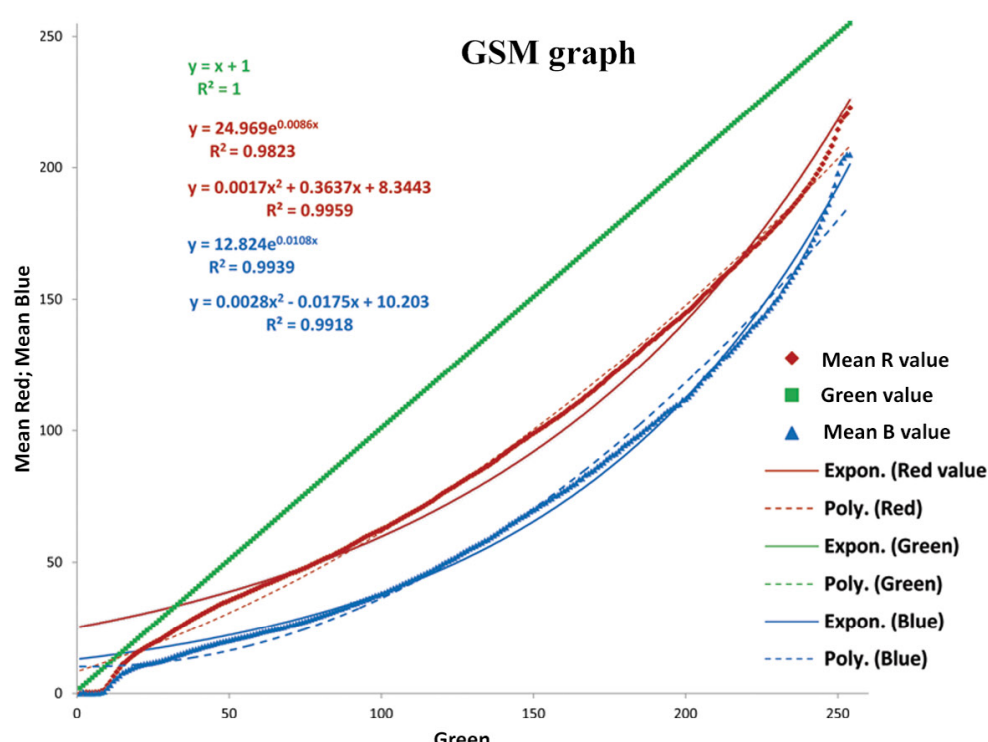

B

Comparison of exponential and polynomial models fitted on GSM mean blue and red values in four monocultures of wheat cultivars (in 6 replicates).

\begin{tabular}{|c|c|c|c|c|c|c|c|c|c|c|c|c|c|c|c|c|c|c|}
\hline \multirow{3}{*}{ Cultivars } & \multicolumn{10}{|c|}{ Consistency of coefficients among replicates of a single cultivar (coefficient of variation, \%C.V.) } & \multicolumn{8}{|c|}{ Goodness of fitness ( $\mathrm{R}^{2}$ and RMSE averaged over replicates of each cultivar) } \\
\hline & \multicolumn{2}{|c|}{ Exponential (red) } & \multicolumn{3}{|c|}{ Polynomial (red) } & \multicolumn{2}{|c|}{ Exponential (blue) } & \multicolumn{3}{|c|}{ Polynomial (blue) } & \multicolumn{2}{|c|}{ Exponential (red) } & \multicolumn{2}{|c|}{ Exponential (blue) } & \multicolumn{2}{|c|}{ Polynomial (red) } & \multicolumn{2}{|c|}{ Polynomial (blue) } \\
\hline & $b$ coeff. & $a$ coeff. & oeff. & $x$ coeff. & Constant & $b$ coeff. & $a$ coeff. & oeff. & $x \mathrm{c}$ & Constant & $\mathrm{R}^{2}$ & RMSE & $\mathrm{R}^{2}$ & $\mathrm{RM}$ & $\mathrm{R}^{2}$ & $\begin{array}{l}\text { Poly. Red } \\
\text { RMSE }\end{array}$ & $B R^{2}$ & B RMSE \\
\hline 1 & 2.842242 & 6.062613 & 14.75875 & 11.05724 & 8.282532 & 2.909595 & 10.42653 & 3.661675 & 42.22079281 & 9.316109 & 0.977036 & 9.201255 & 0.994802 & 3.743326 & 0.995735 & 3.839738529 & 0.989462419 & 5.152881425 \\
\hline 2 & 2.980015 & 6.363184 & 14.82473 & 13.03087 & 38.96945 & 2.690594 & 9.137798 & 10.79274 & 88.67124038 & 40.3738 & 0.978714 & 8.855079 & 0.994862 & 3.807087 & 0.995356 & 4.013751066 & 0.99092375 & 4.968749335 \\
\hline 3 & 1.942587 & 3.895648 & 14.74602 & 9.836061 & 14.49161 & 1.972454 & 7.783508 & 2.808954 & 45.24099172 & 12.54156 & 0.97608 & 9.859379 & 0.994332 & 4.551637 & 0.996347 & 3.668294207 & 0.99524384 & 3.878802089 \\
\hline 4 & 2.747959 & 6.519684 & 18.04506 & 15.85442 & 20.00396 & 2.08405 & 5.87855 & 7.170945 & 47.40564927 & 20.73622 & 0.977735 & 8.816504 & 0.991632 & 4.55033 & 0.993608 & 4.575723614 & 0.983548832 & 6.369765135 \\
\hline
\end{tabular}

The formulas of exponential and polynomial (quadratic) equations used are the simple forms of $y=a e^{b x}$ and $y=a x^{2}+b x+c$, respectively; e.g. for red color: Red $=a . e^{b . G r e e n}$ and Red $=a(\text { Green })^{2}+b($ Green $)+C$.

Figure S1. Comparison of the exponential and polynomial equations of the GSM curves (green-gradient based segmentation method), estimated for monocultures of 4 wheat cultivars (in 6 replicates). A. The GSM graph of a monoculture plot of the $1^{\text {st }}$ cultivar at the late booting phase. The exponential and polynomial models are fitted to the mean red and mean blue values of GSM. B. Coefficients of variation (\%C.V. s) of the coefficients of the exponential and polynomial equations and the fitness measurers calculated for 6 replicates of the monocultures of wheat cultivars. 
bioRxiv preprint doi: https://doi.org/10.1101/241786; this version posted January 13, 2019. The copyright holder for this preprint (which was not certified by peer review) is the author/funder. All rights reserved. No reuse allowed without permission.
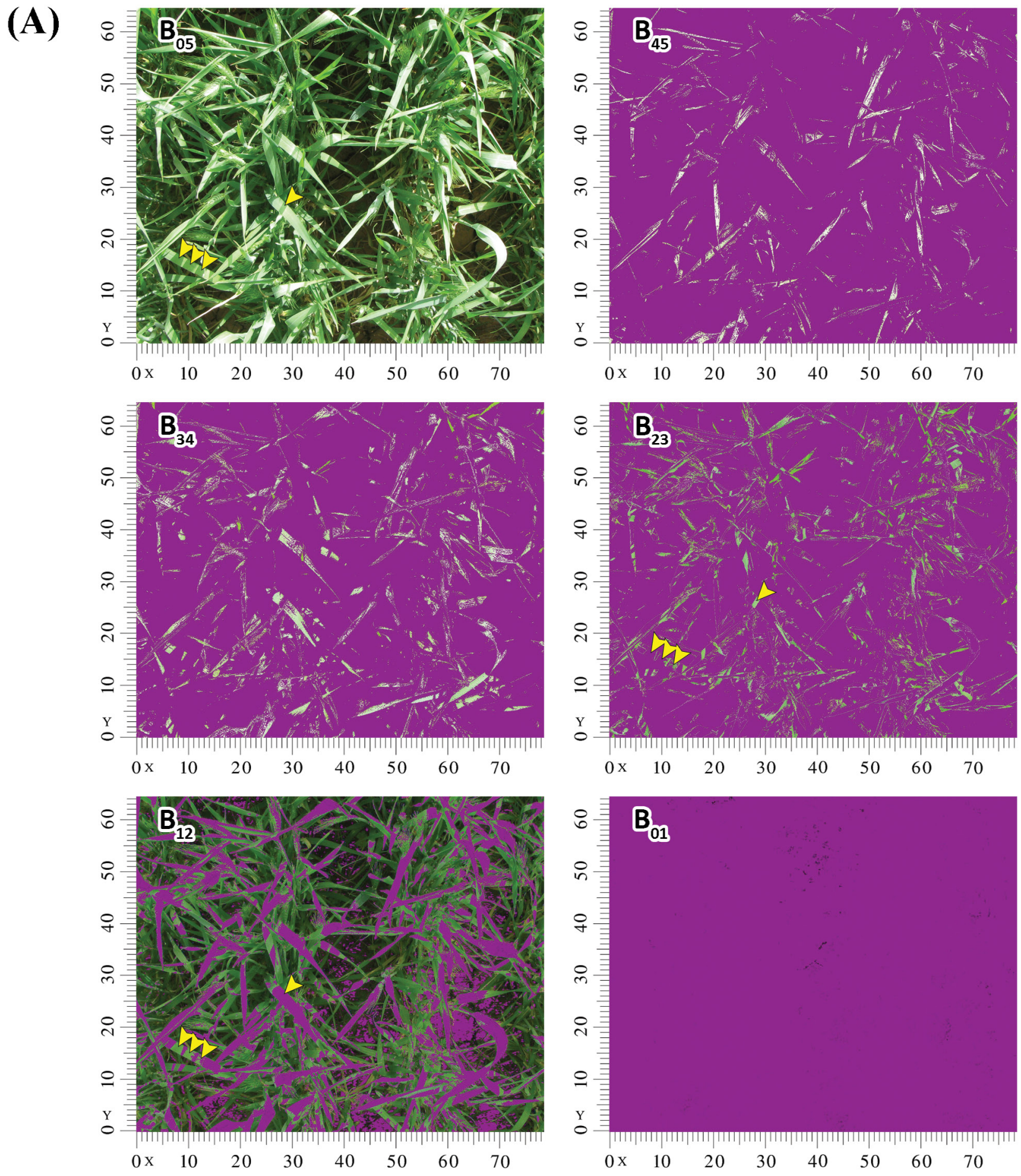

Figure S2. Segmentation type $3\left(\mathrm{ST}_{3}\right)$ for the image of a wheat canopy (monoculture of the $1^{\text {st }}$ cultivar) at booting stage. (A) The central section of an image segmented based on $\mathrm{ST}_{3}$ of the blue curve (depended on variations in the curve local slopes relative to the green trend). In each figure, the purple mask has covered the excluded pixels. Compare the shadow on the leaf in the position $(x=28, y=26)$ with the three parallel strips of shadows on the leaf at $(x=10, y=15)$. The first one is categorized as a partial shadow, since it is observable in the segment $B_{23}$ (where the local slopes of the blue curve are almost equal to the slope of green trend, i.e. 1), while, the second set is recognized as complete shadow $\left(B_{12}\right.$, i.e. the local slopes of the blue curve are lower than 1). Similarly, different parts of any single rolled or folded leaf in various segments may be tracked. Notably, (i) non-vegetative pixels are excluded previously by the segmentation type 1 ( $\mathrm{ST}_{1}$ ), and (ii) the 5 segments (classes) demonstrated here are complementary to each other, so each pixel may be included only in one segment. (B) The relative variations in local slopes of the red and blue GSM curves, compared to the green line. $\mathrm{ST}_{3}$ is developed based on this graph; see Fig. 1 for details.

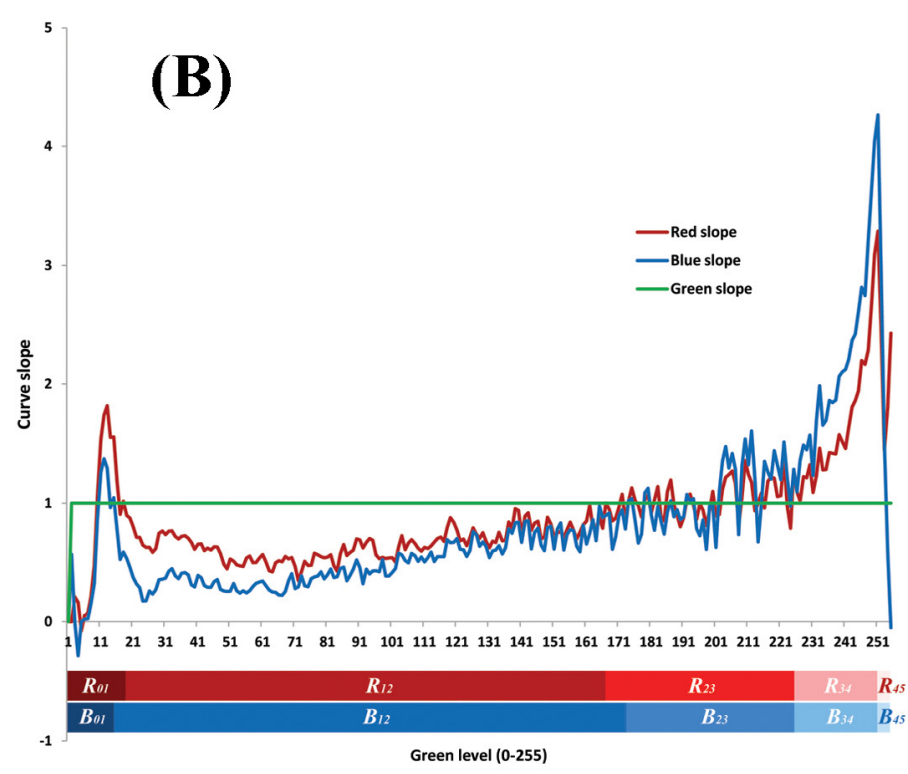


bioRxiv preprint doi: https://doi.org/10.1101/241786; this version posted January 13,2019 . The copyright holder for this preprint (which was not certified by peer review) is the author/funder. All rights reserved. No reuse allowed without permission.

A

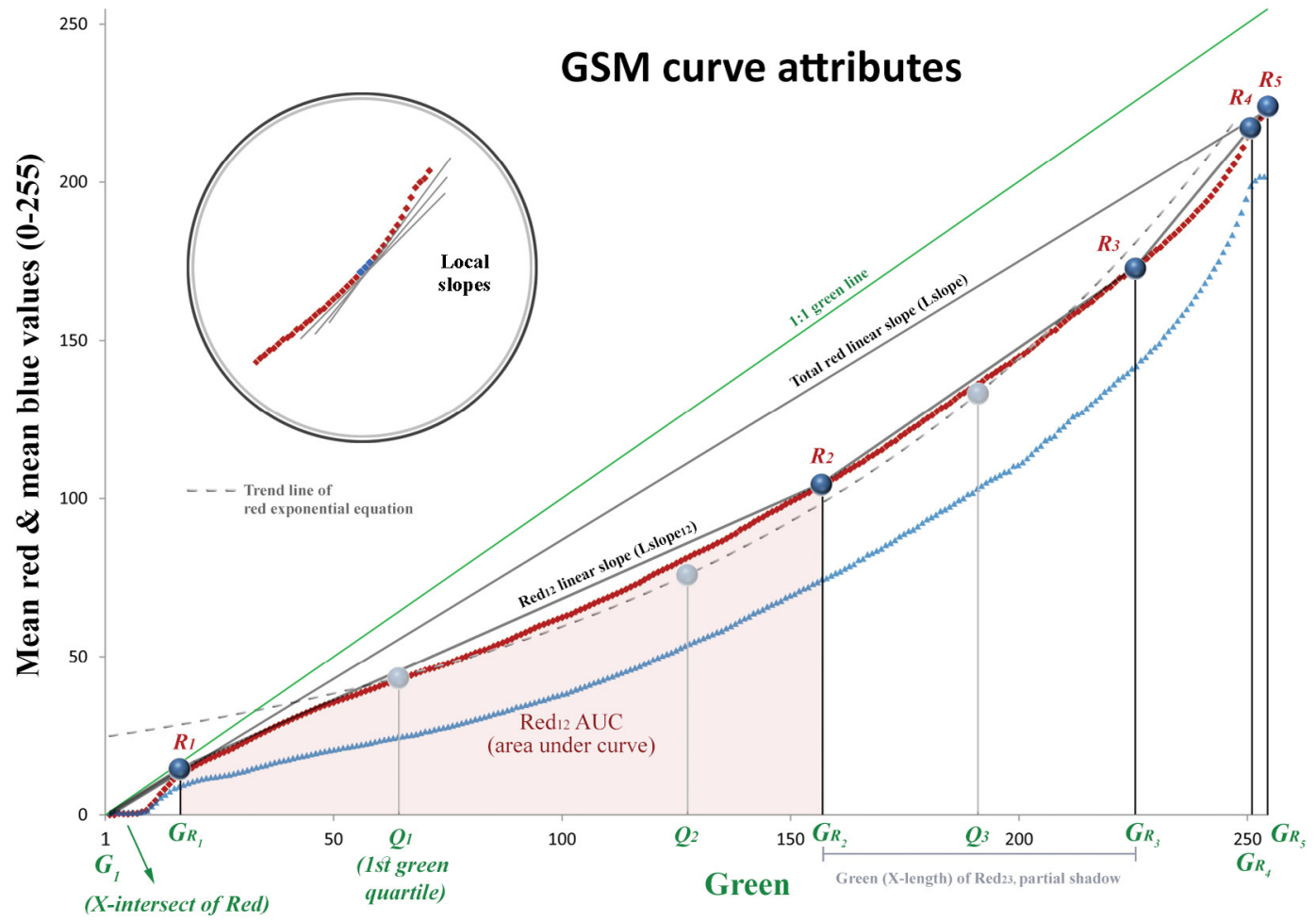

B

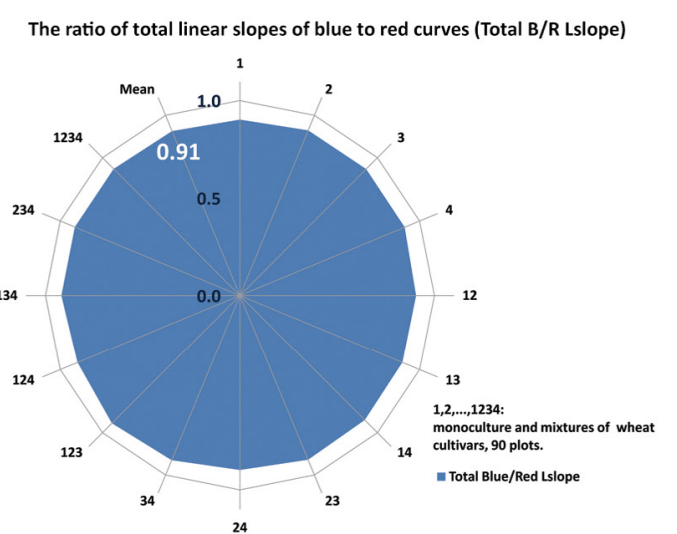

E

Comparative \%X-intercepts of GSM red and blue curves, which shows the blue curve reaches zero 10 times earlier than the red curve,

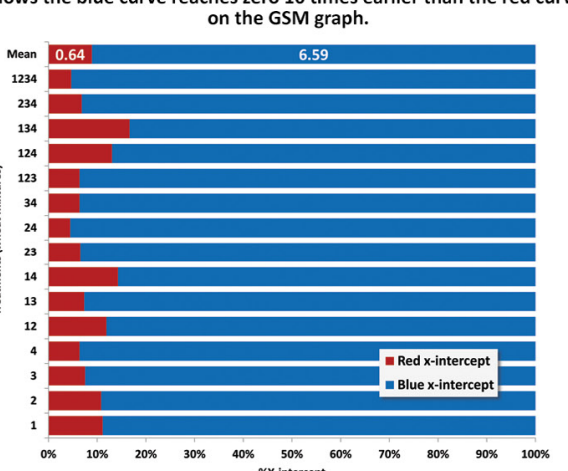

C

Mean linear slopes of red and/or blue curves in different $\mathrm{ST}_{3}$ classes

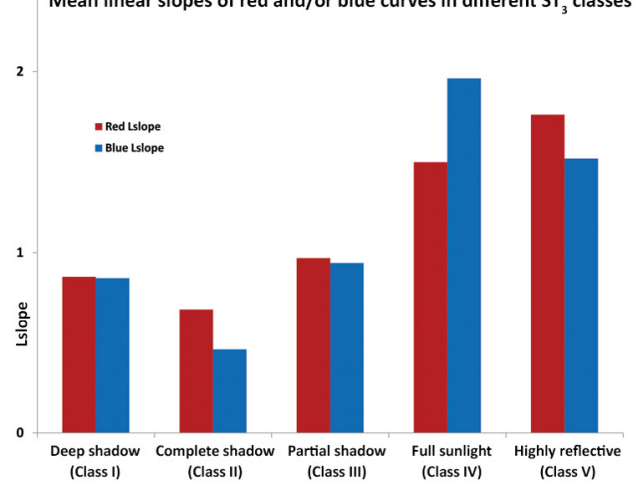

$\mathbf{F}$

Negative correlation between the " $a$ " and " $b$ "coefficients of the exponential equation fitted to the GSM red curve

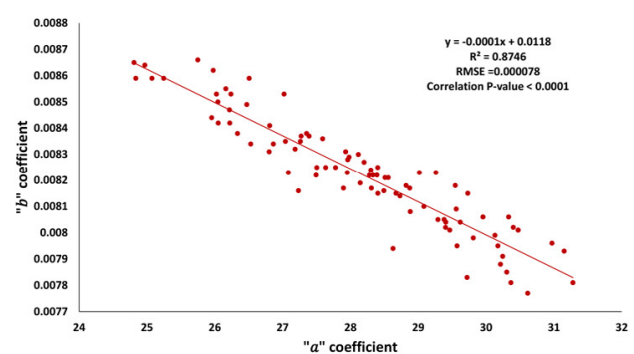

D

The ratio of mean local slopes of red to blue curves, under the highly reflective and full sunlight segments

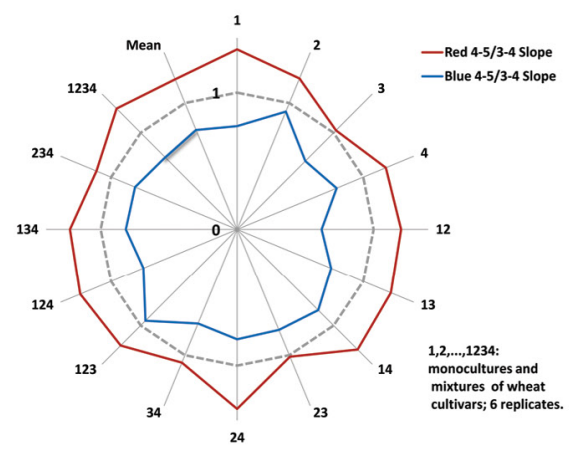

G

Negative correlation between the " $a$ " and " $b$ "coefficients of the exponential equation fitted to the GSM blue curve

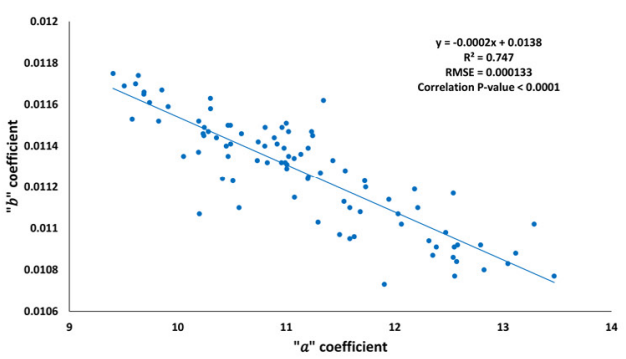

Figure S3. GSM-curve attributes. (A) Schematic illustration of GSM-curve attributes of the red curve. (B) to (G): several considerable aspects revealed evaluating curve attributes. Treatments $(1,2,3,4,12,13,14,23,24,34$, $123,124,134,234,1234)$ are 15 mono- and mixed cropping of wheat cultivars in 6 replicates ( 1 to 4 are early to middle ripening cultivars, and each digit shows a cultivar included in the mixture). 
A

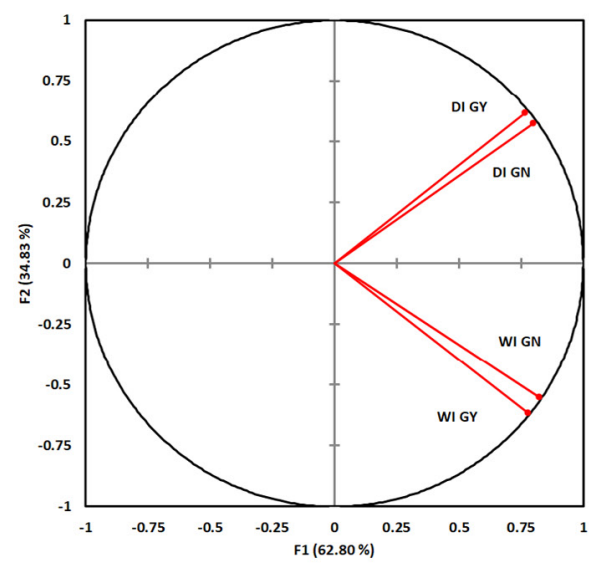

C
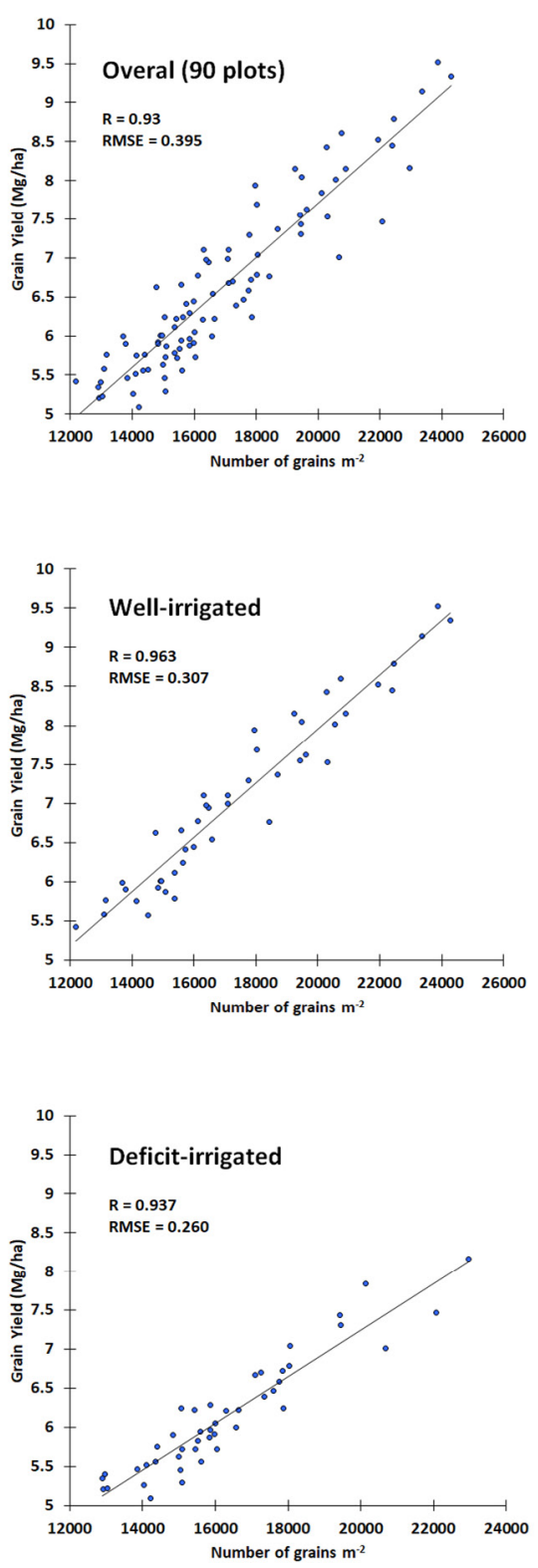

B

Effect of post-anthesis deficit irrigation on GY and GN.

\begin{tabular}{llll}
\hline & GN & GY & No. of plots \\
\hline WI & 17527.182 & 7.099 & 45 \\
DI & 16292.076 & 6.143 & 45 \\
\%Reduction & 7.046803074 & 13.46668545 & - \\
P-value & 0.019 & $<000$ & - \\
\hline
\end{tabular}

WI, DI, GN, and GY are well- and deficit-irrigated, No. of grains $\mathrm{m}^{-2}$, and grain yield $(\mathrm{Mg} / \mathrm{ha})$, respectively. Values of $\mathrm{GN}$, $\mathrm{GY}$, and \%Reduction are averaged over the corresponding 45 plots. P-values show the effects of deficit-irrigation.

D
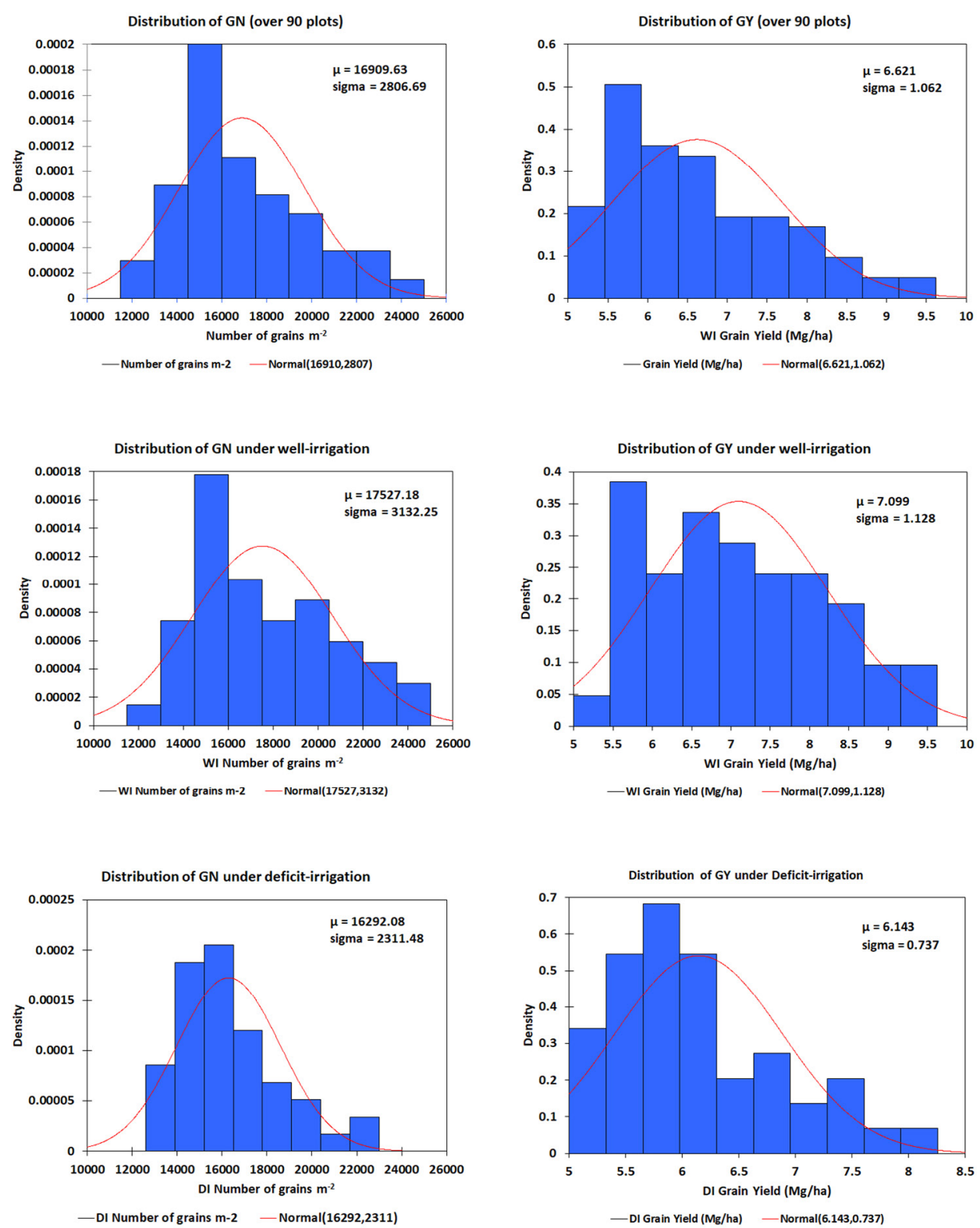

Figure S4. Effect of post-anthesis deficit irrigation (with 50\% of evapotranspiration demand) on grain yield (GY) and number of grains $\mathrm{m}^{-2}$ of the experimental plots. (A) Results of Principal Component Analysis (PCA), (B) A brief results of ANOVA and mean comparison of GY and GN values as affected by the post-anthesis deficit irrigation. (C) The relationship between GY and GN under various conditions. (D) Distribution of GY and GN values under different conditions; the red trend indicates the normal distribution. It is notable that in all of the 6 charts, the samples followed normal distribution (P-value $>0.05$ ). 


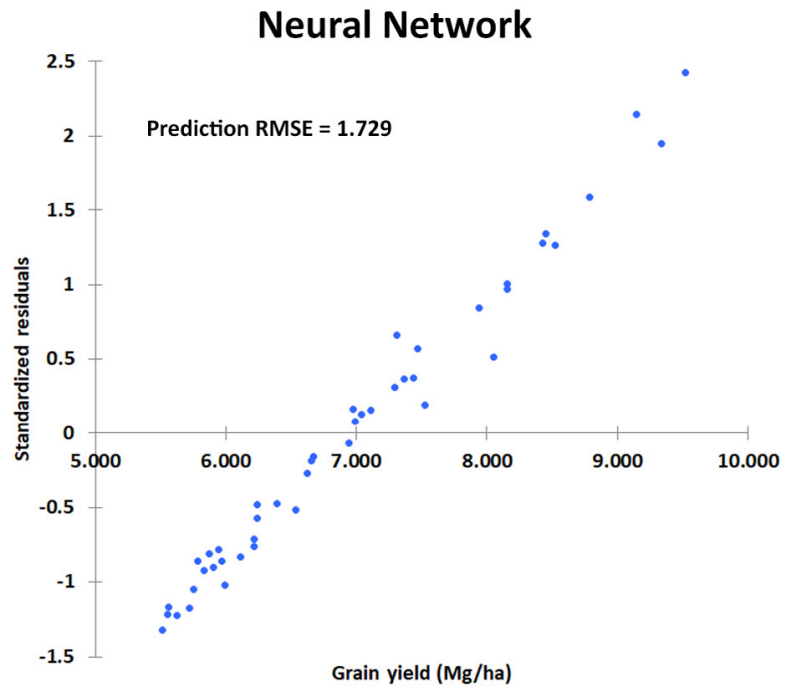

Generalized Linear Model
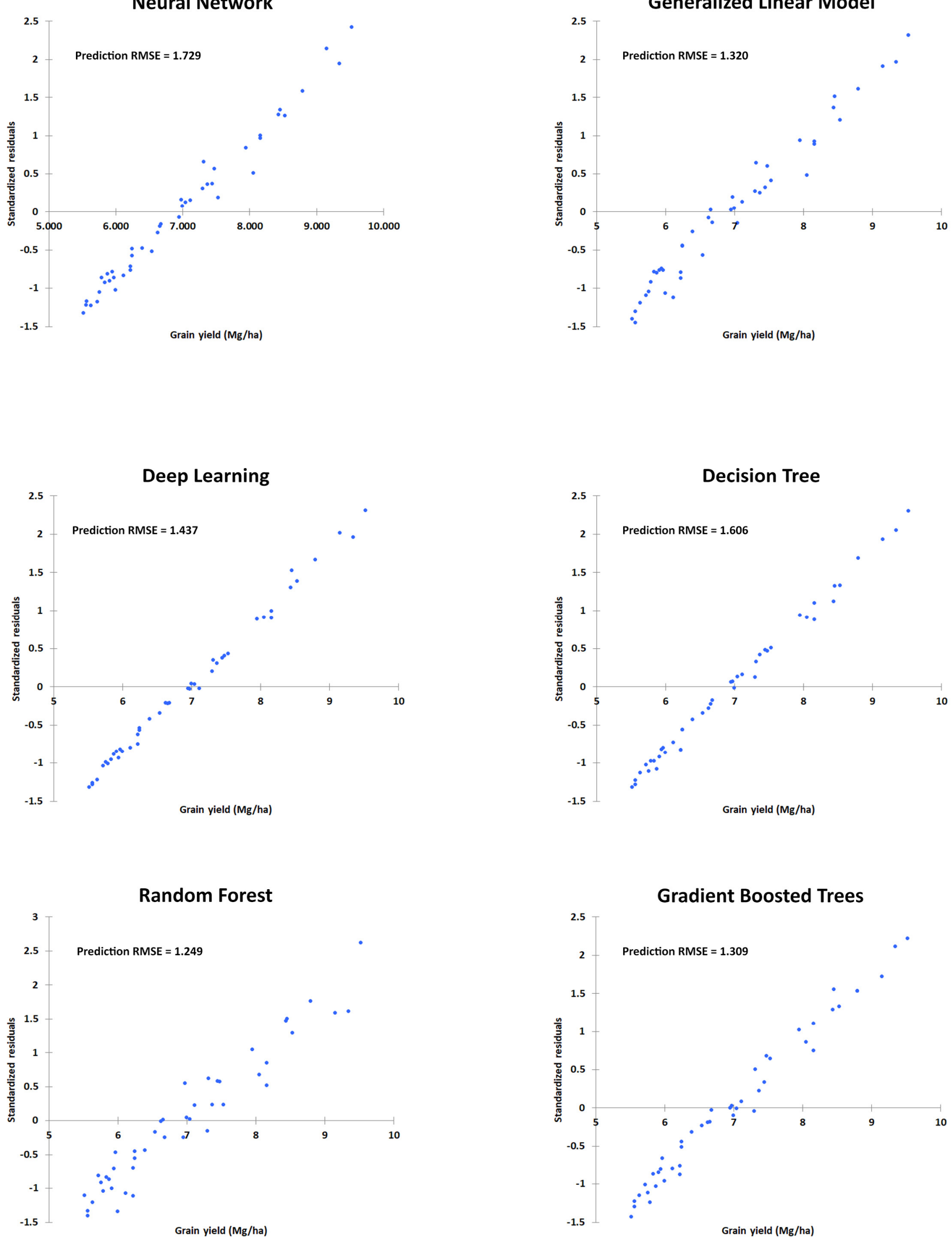

Figure S5. Standardized residuals of the GSM-based grain yield predictions by various models using the $1^{\text {st }}$ computation approach (i.e. the quantitative values of grain yield were used). Apparently the residuals were minimum almost at $\mathrm{GY}=7 \mathrm{Mg} / \mathrm{ha}$. 
bioRxiv preprint doi: https://doi.org/10.1101/241786; this version posted January 13,2019 . The copyright holder for this preprint (which was not certified by peer review) is the author/funder. All rights reserved. No reuse allowed without permission.

Neural Network

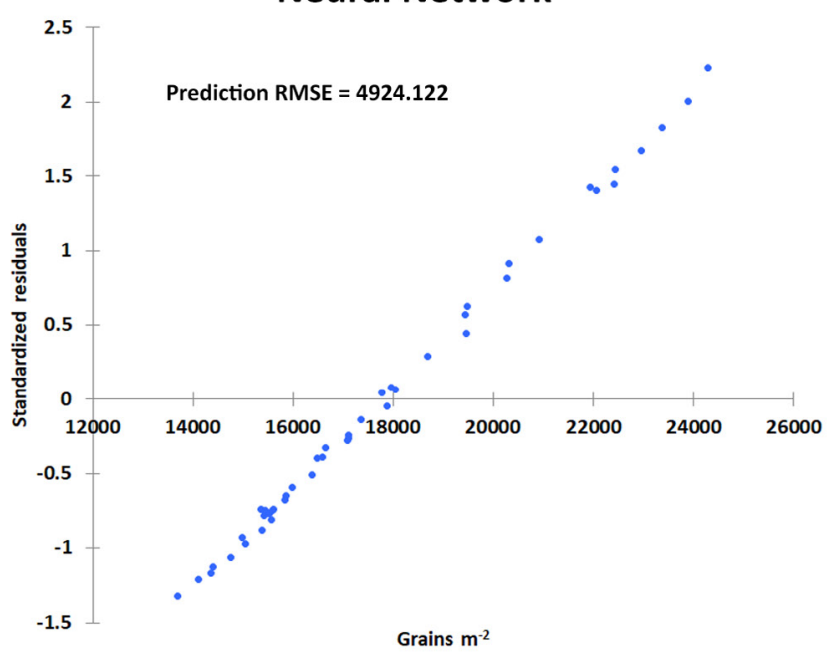

Deep Learning

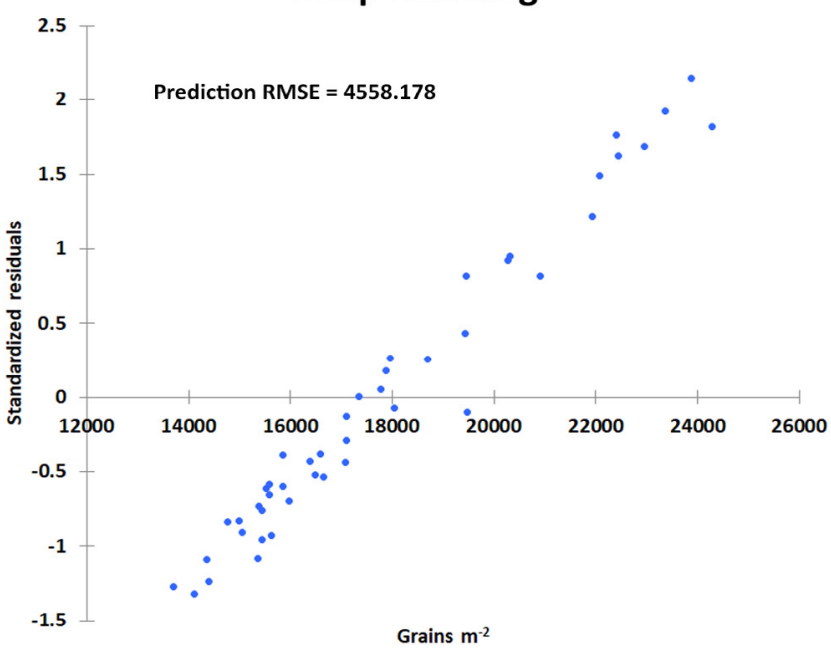

Random Forest

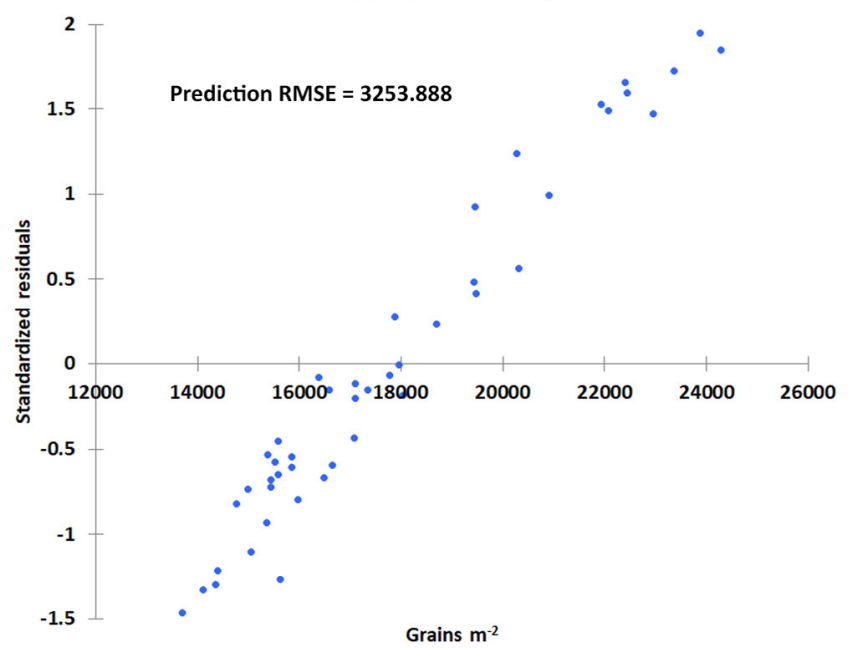

Generalized Linear Model

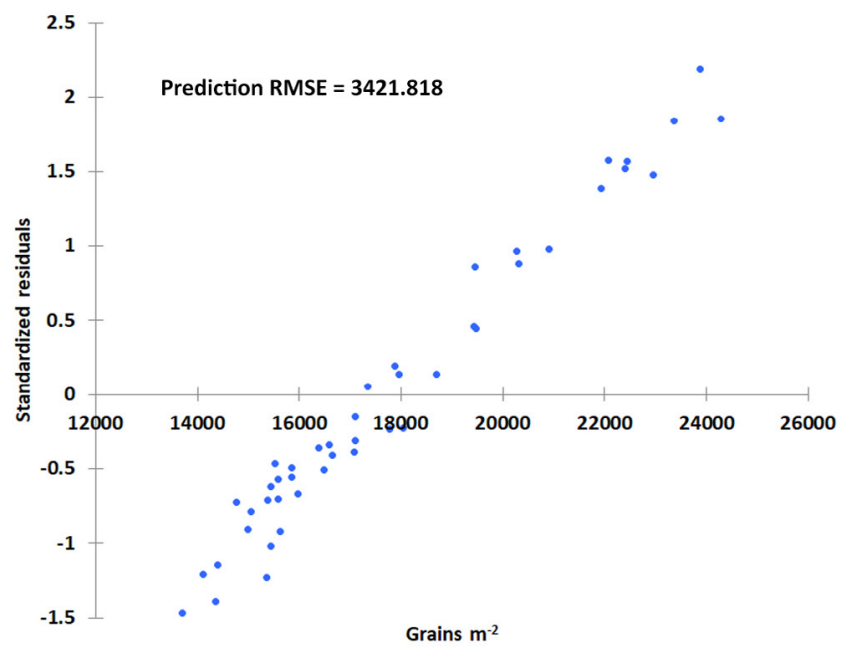

Decision Tree

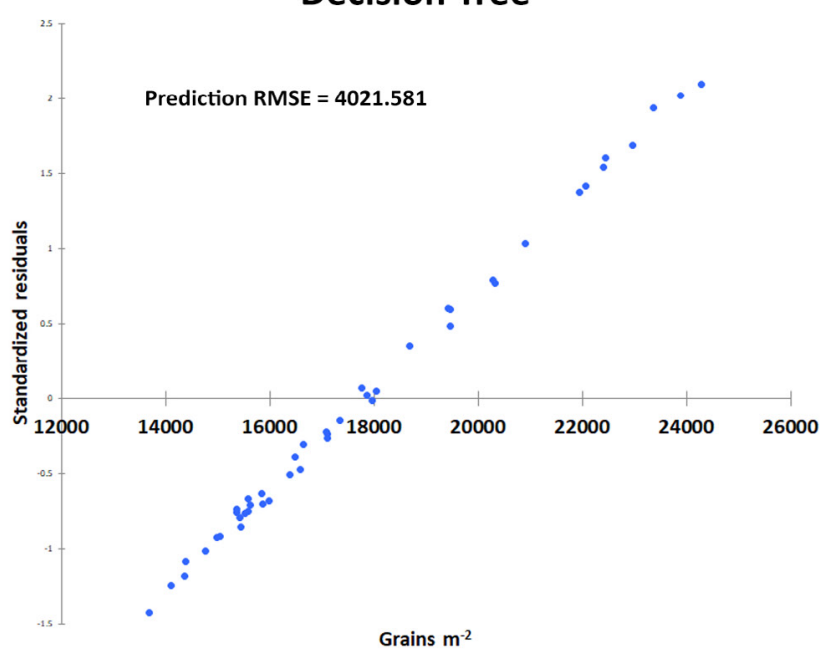

Grains $\mathrm{m}^{-2}$

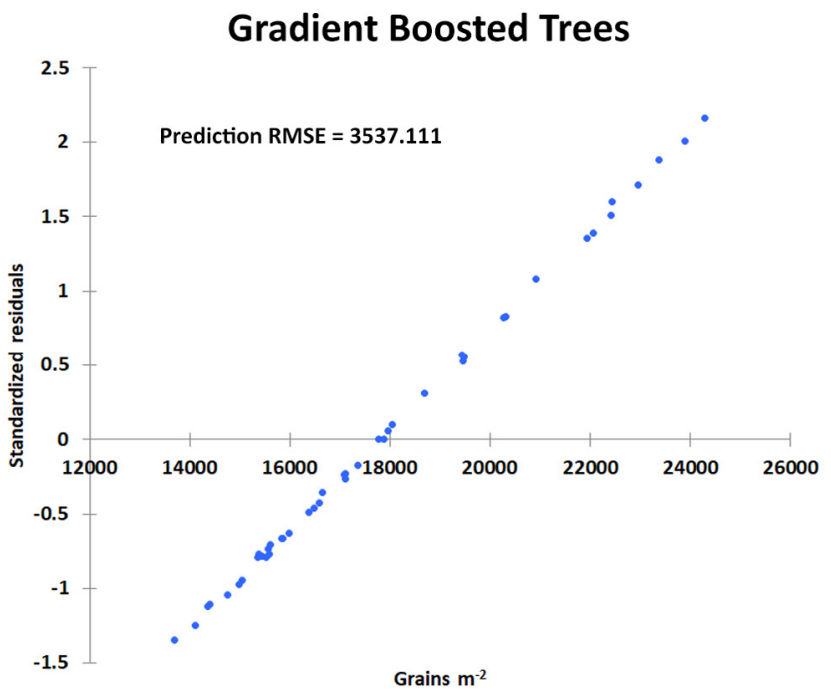

Figure S6. Standardized residuals of the GSM-based predictions of number of grains $\mathrm{m}^{-2}$ by various models using the $1^{\text {st }}$ computation approach (i.e. the quantitative values of grain yield were used). The minimum residuals were observed almost at 18000 grains $\mathrm{m}^{-2}$. 


\section{High yielding}
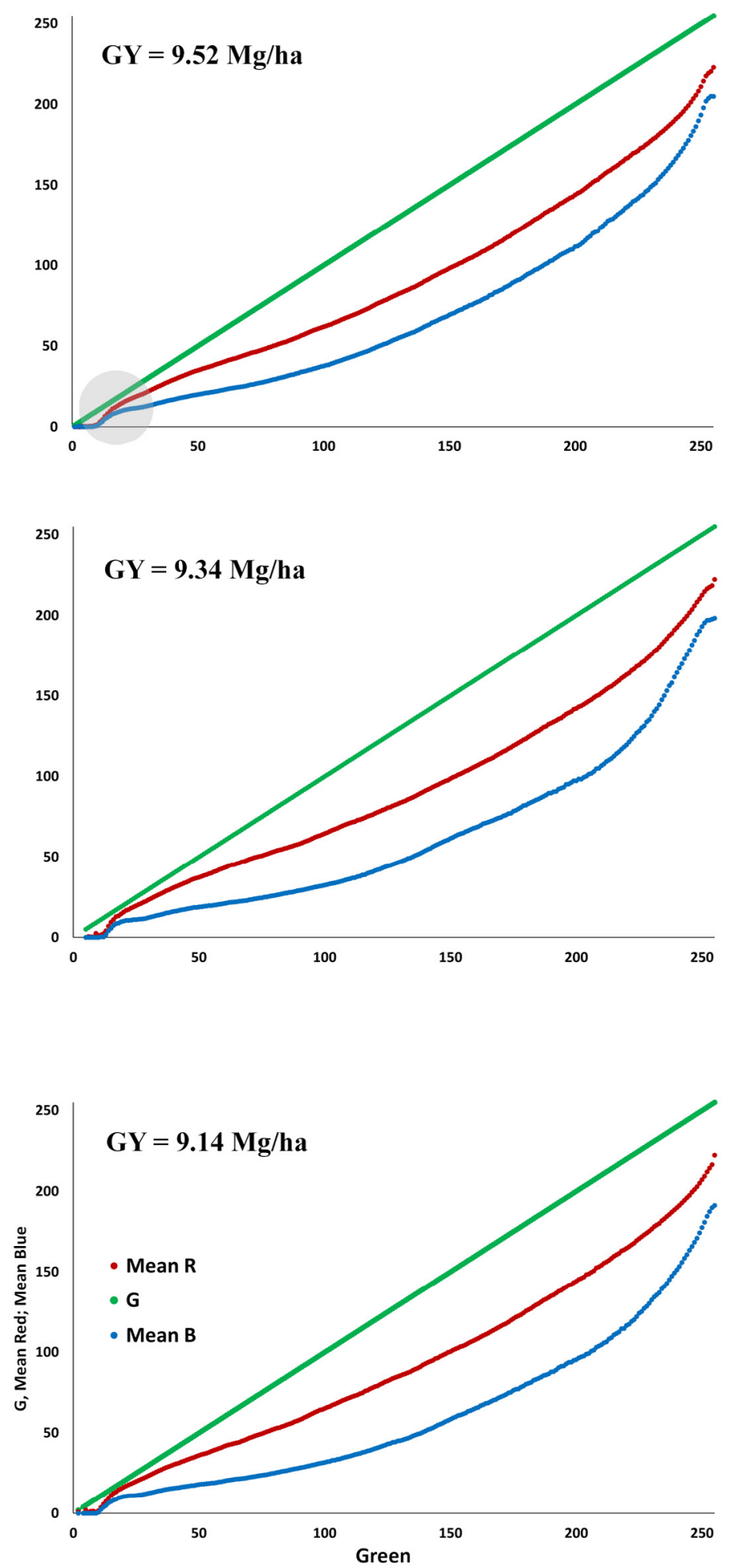

\section{Low yielding}
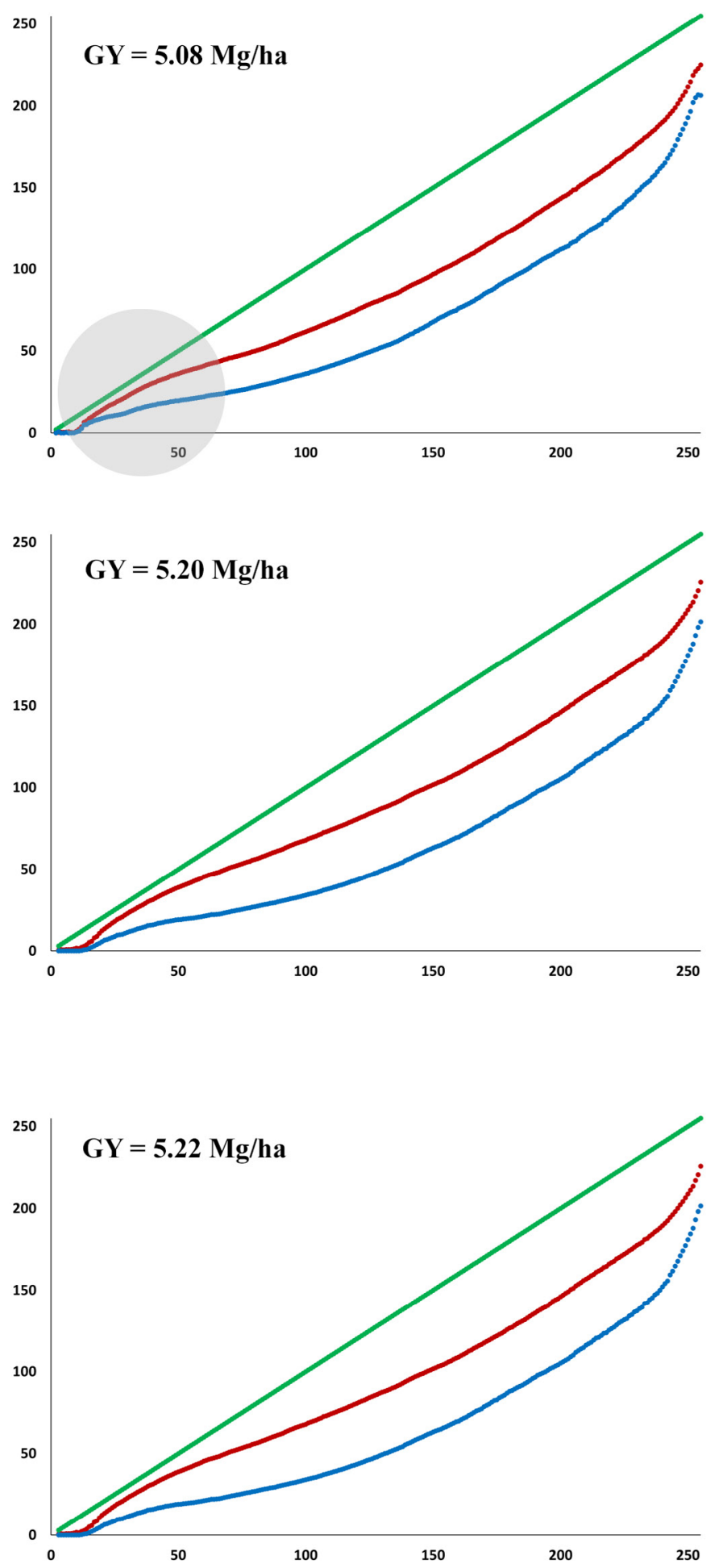

Figure S7. Comparison of the GSM graphs of high- vs. low-yielding plots. The three graphs at the left side belongs to the plots those produced the highest GYs (grain yields; i.e. in average $9.33 \mathrm{Mg} / \mathrm{ha}$ ), and the three ones at the right side show the lowest yielding plots (with the averaged grain yields of $5.17 \mathrm{Mg} / \mathrm{ha}$ ). It seems that visually distinguishing of these two yielding types from their GSM graphs is difficult and requires quantifying approaches including the data mining analyses of the curve-derived characteristics, as described in the manuscript. However, some symptoms may be visually recognizable -as for instance- the very first major curvature change of the curves (particularly for the red curve) in the low yielding samples seems to be occurs in a wider range on the graph, compared with the high-yielding ones (grey filled circles). Validation of such observations also needs further studies. 
bioRxiv preprint doi: https://doi.org/10.1101/241786; this version posted January 13, 2019. The copyright holder for this preprint (which was not certified by peer review) is the author/funder. All rights reserved. No reuse allowed without permission.

I

47 DAS

(days after sowing)

(Early tillering)

II

93 DAS

(Tillering)

III

151 DAS

(Early stem elongation)

IV

194 DAS

(Grain filling)
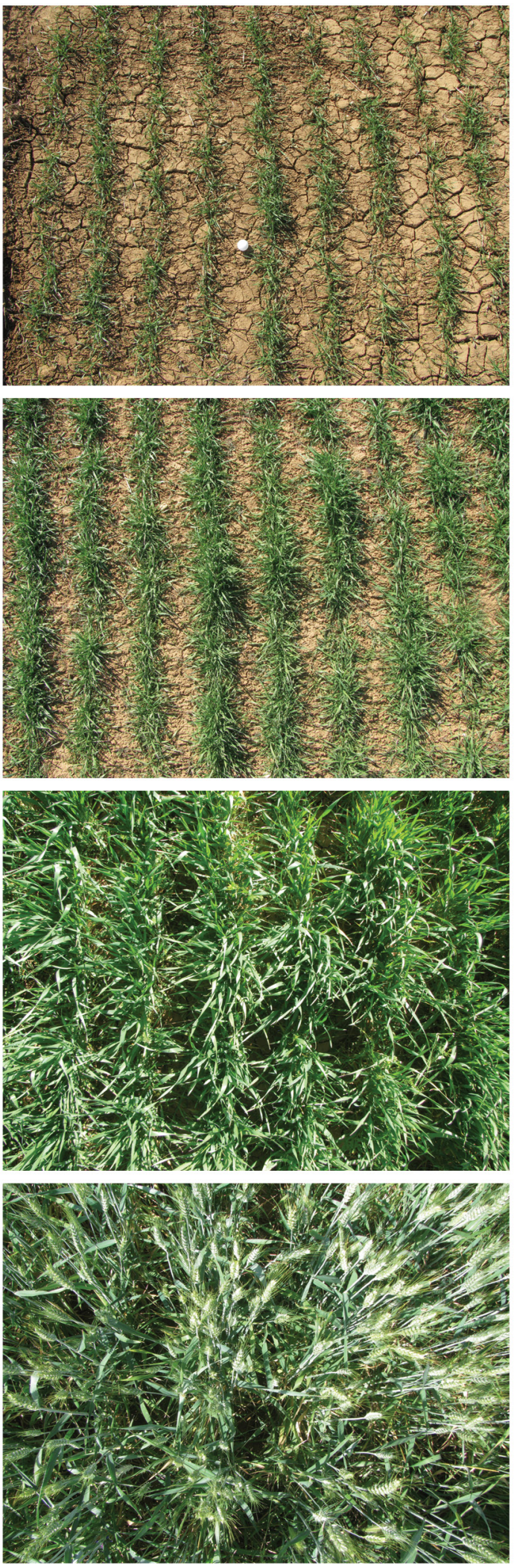

V

197 DAS

(Grain filling)

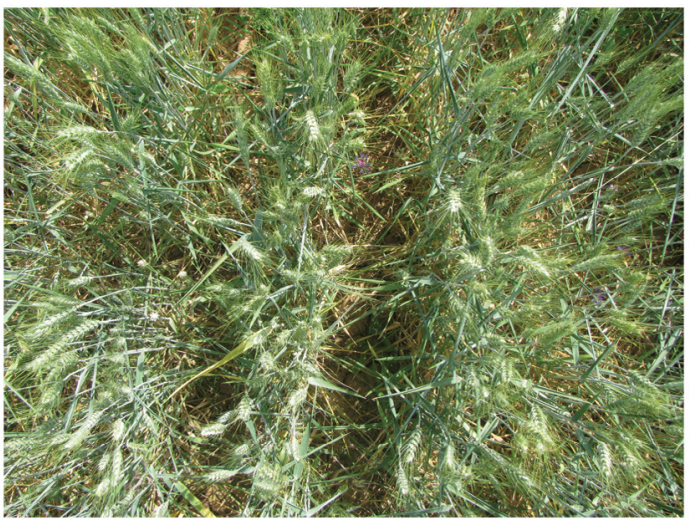

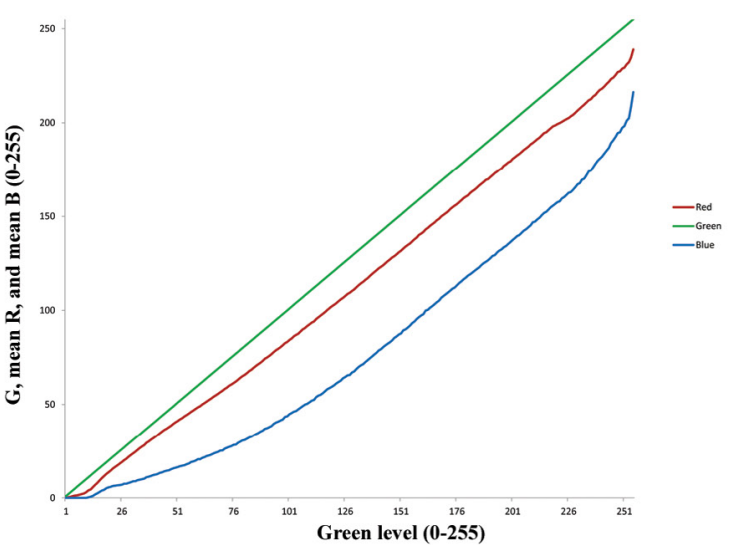
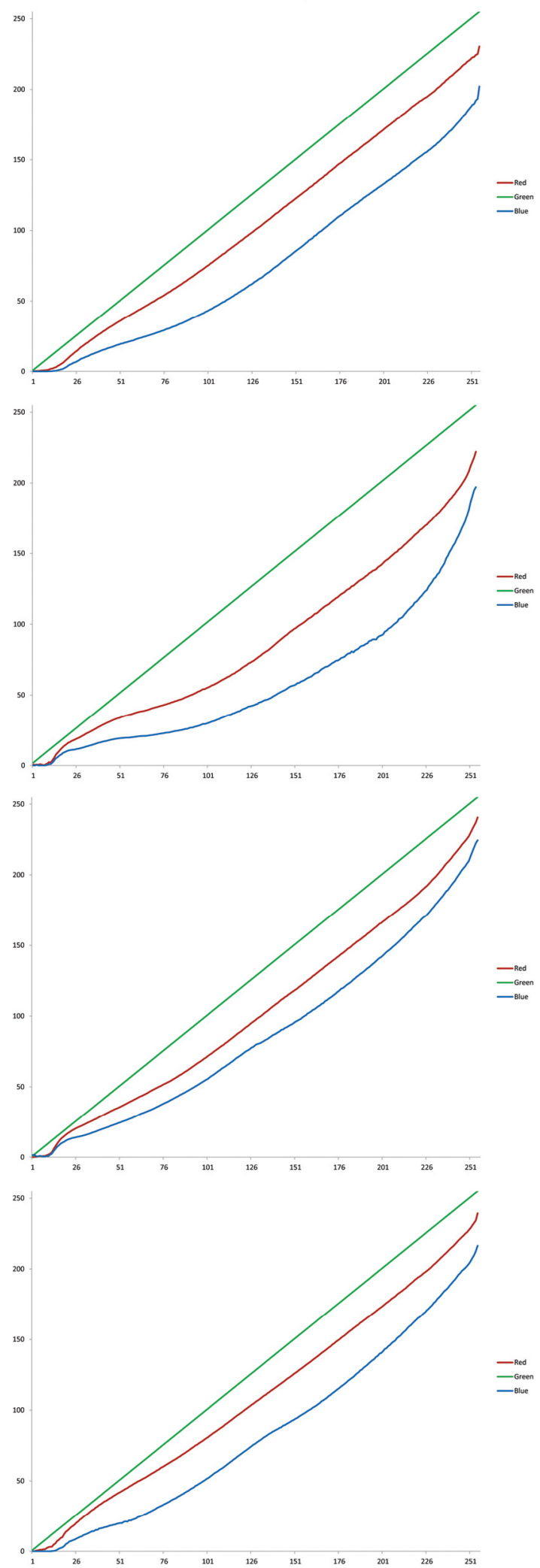

Figure S8. Effect of growth stage (phenology) on GSM graph in a green monoculture canopy. Consider the variations of the red and blue curves relative to green, which in the most dense canopy at the stage (III) show the highest degrees of curvature. 
A VI 208 DAS (Late physiological ripening)

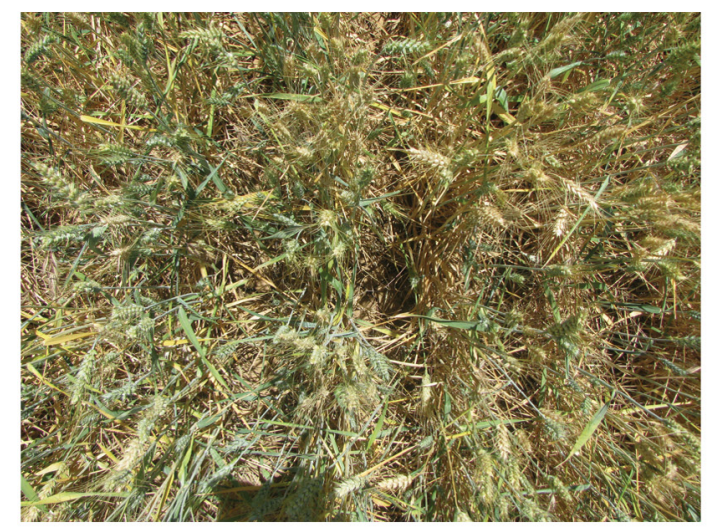

VII 211 DAS

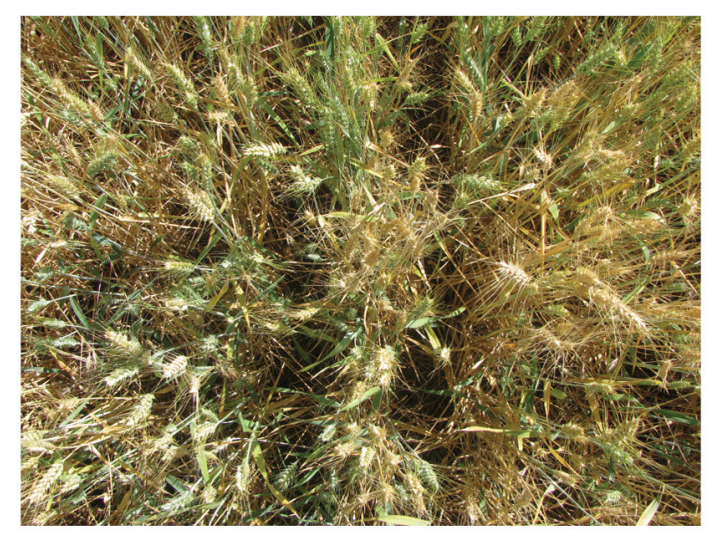

VIIII 214 DAS

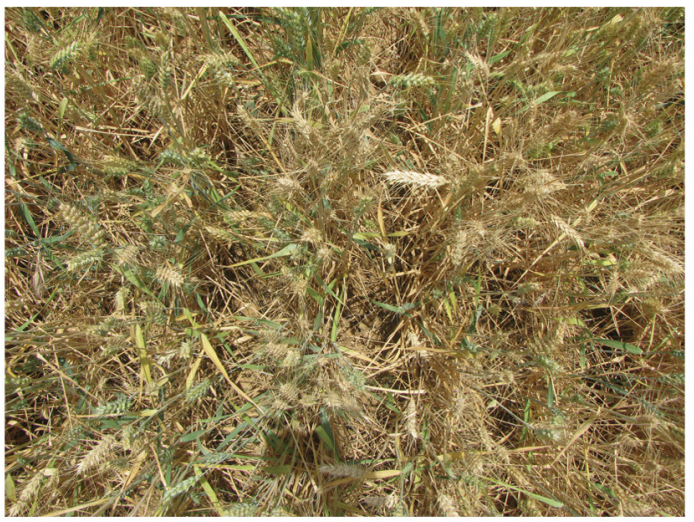

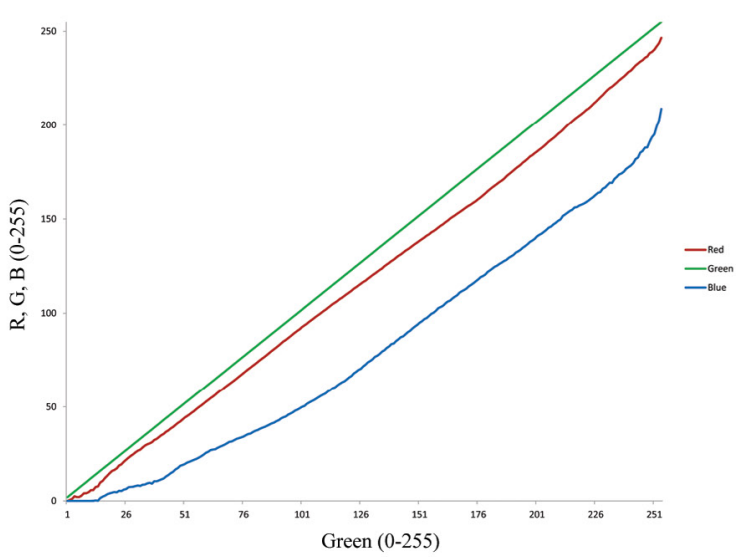
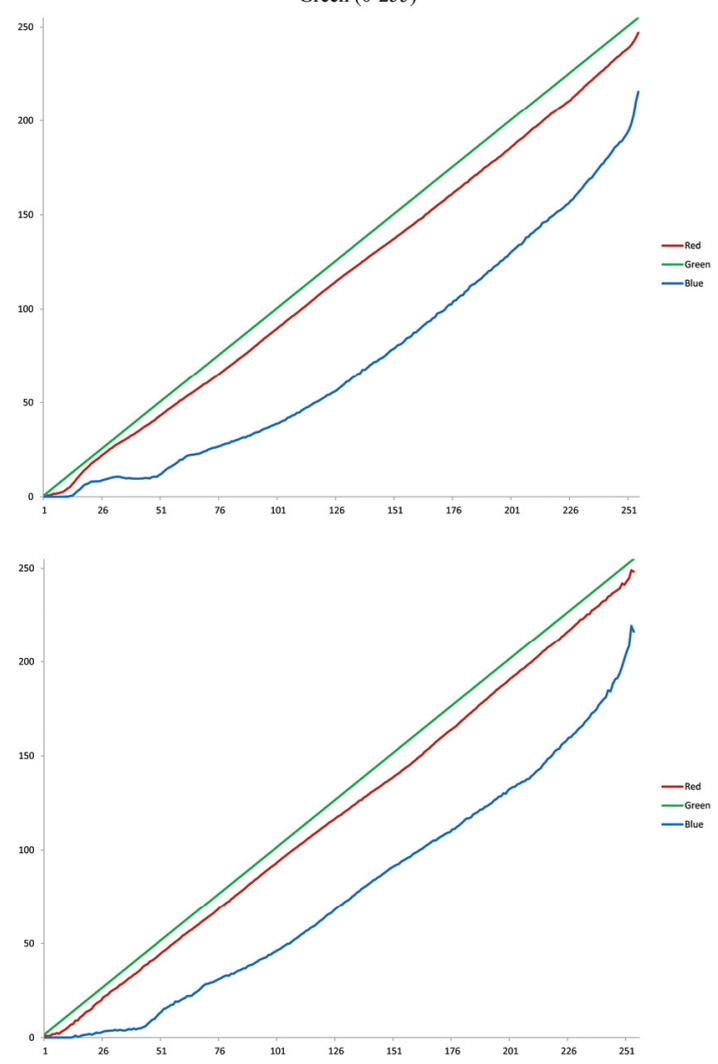

B

\begin{tabular}{lllllllllllll}
$\begin{array}{l}\text { Days after } \\
\text { sowing }\end{array}$ & $\begin{array}{l}a \text { - coefficient } \\
\text { of R equation }\end{array}$ & $\begin{array}{l}a \text { - coefficient } \\
\text { of equation }\end{array}$ & $\begin{array}{l}b \text { - coefficient } \\
\text { of equation }\end{array}$ & $\begin{array}{l}b \text { - coefficient } \\
\text { of B equation }\end{array}$ & $\mathrm{R} R^{2}$ & $\begin{array}{l}\mathrm{R} \\
R M S E\end{array}$ & $\mathrm{~B} R^{2}$ & $\begin{array}{l}\mathrm{B} \\
R M S E\end{array}$ & $\begin{array}{l}\mathrm{GR} \\
\text { NABC }\end{array}$ & $\begin{array}{l}\mathrm{GB} \\
\text { NABC }\end{array}$ & $\begin{array}{l}\mathrm{RB} \\
\text { NABC }\end{array}$ & \begin{tabular}{l}
$\%(\mathrm{GR} / \mathrm{GB})$ \\
\hline 47
\end{tabular} \\
\hline 36.025 & 16.905 & 0.008 & 0.010 & 0.951 & 15.886 & 0.974 & 10.500 & 0.13 & 0.39 & 0.27 & 32.24 \\
151 & 31.672 & 17.251 & 0.008 & 0.010 & 0.954 & 15.018 & 0.972 & 10.176 & 0.18 & 0.41 & 0.23 & 44.71 \\
194 & 22.693 & 9.181 & 0.009 & 0.012 & 0.983 & 7.937 & 0.993 & 3.942 & 0.32 & 0.55 & 0.23 & 58.45 \\
197 & 29.871 & 21.119 & 0.008 & 0.009 & 0.969 & 12.251 & 0.980 & 9.049 & 0.20 & 0.33 & 0.13 & 60.45 \\
208 & 34.420 & 19.353 & 0.008 & 0.010 & 0.959 & 14.096 & 0.975 & 10.475 & 0.15 & 0.35 & 0.20 & 42.75 \\
211 & 38.556 & 19.453 & 0.008 & 0.009 & 0.951 & 16.136 & 0.964 & 11.992 & 0.09 & 0.37 & 0.29 & 23.18 \\
214 & 38.418 & 14.373 & 0.008 & 0.011 & 0.951 & 16.219 & 0.982 & 8.474 & 0.08 & 0.43 & 0.34 & 19.76 \\
\hline Mean & 38.981 & 16.720 & 0.008 & 0.010 & 0.951 & 16.567 & 0.967 & 11.560 & 0.07 & 0.40 & 0.33 & 17.95 \\
\hline C.V. & 33.830 & 16.794 & 0.008 & 0.010 & 0.959 & 14.264 & 0.976 & 9.521 & 0.15 & 0.40 & 0.25 & 37.44 \\
\hline
\end{tabular}

C

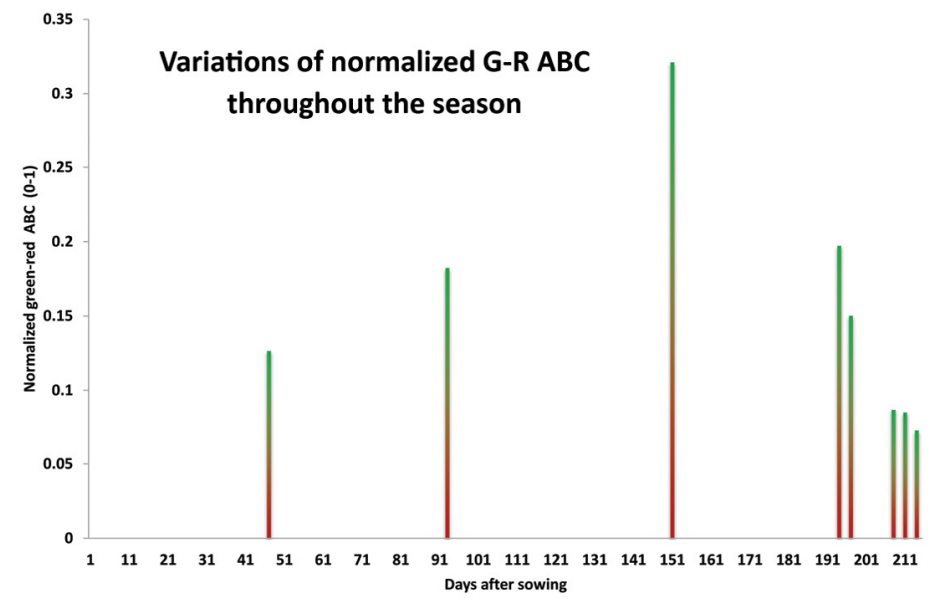

The least values $\square$ The highest values

Figure S9. GSM graph of a ripening canopy, which is also compared with previous stages. (A) GSM seems to be capable to track the canopy status even in a semi-dried stand. Consider the shape of red curve which is approached to the green trend line, late in the season. Obviously, only green pixels are included in the GSM analyses. (B) Various phenological stages are compared using the exponential equations and curve attributes. R, G, and B are red, green, and blue lights; NABC is normalized area between curves, which is calculated by dividing each $\mathrm{ABC}$ value to the total area under green line. (C) GR-NABC is proposed for determining the canopy growth stage, and tracking of ripening. 
Under relatively high diurnal evapotranspiration (ETc) (after several windy days)
Under moderate (usual)

diurnal ETc

(3 calm days after irrigation) 1st cultivar (treatment 1)
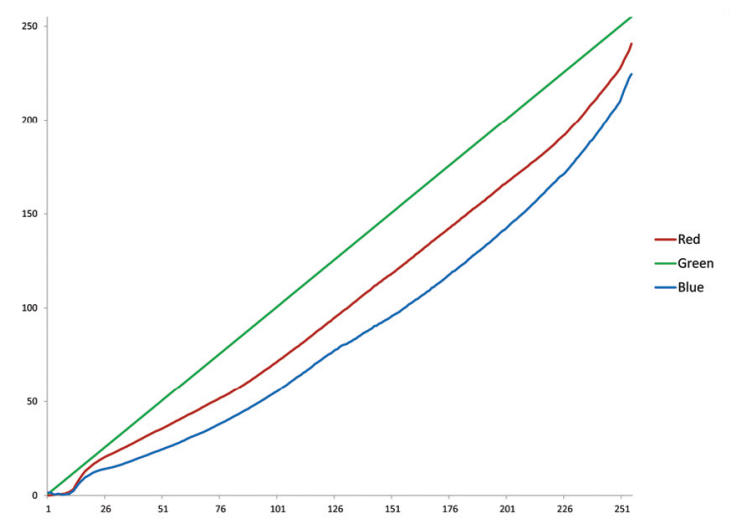

2nd cultivar (treatment 2)
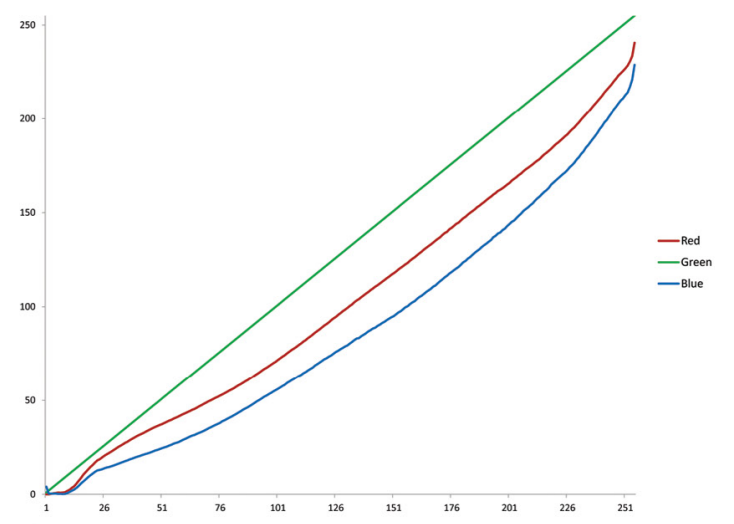

3rd cultivar (treatment 3)

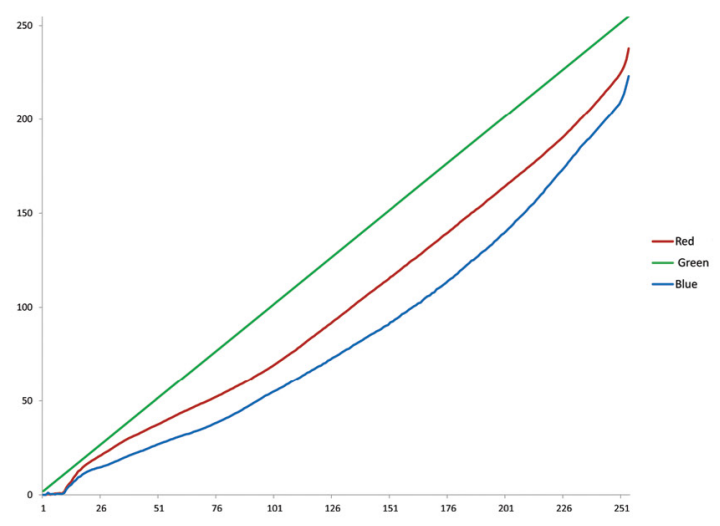

4th cultivar (treatment 4)

i.e. relatively the most sensitive cultivar, or probably, having the least degrees of osmatic adjustment.

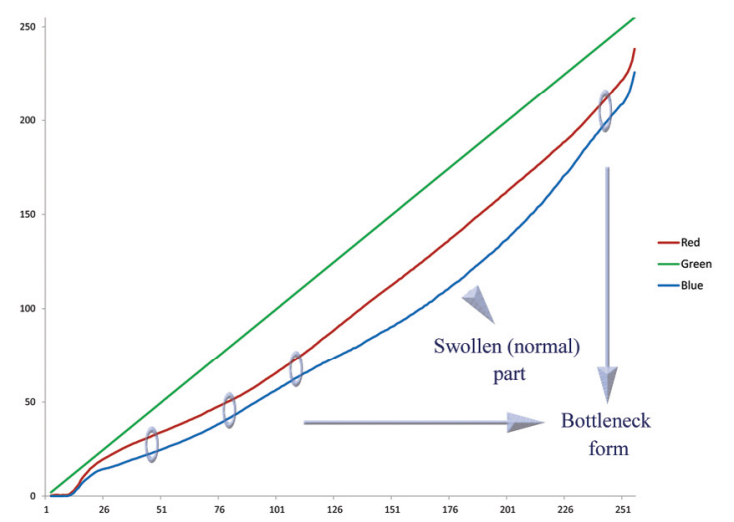

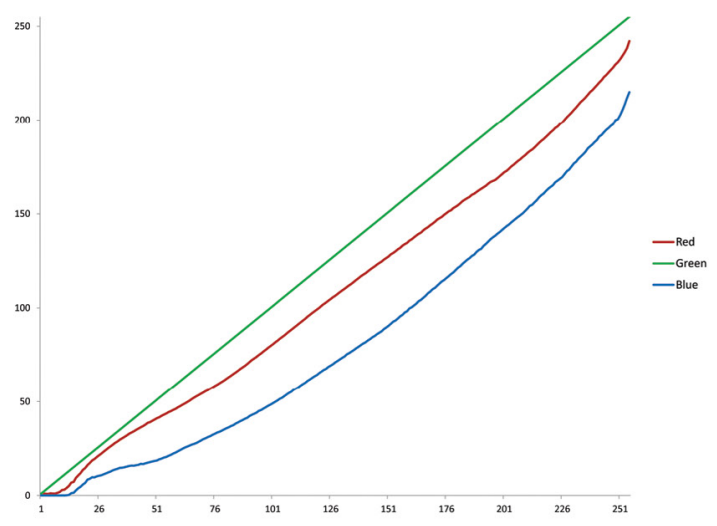
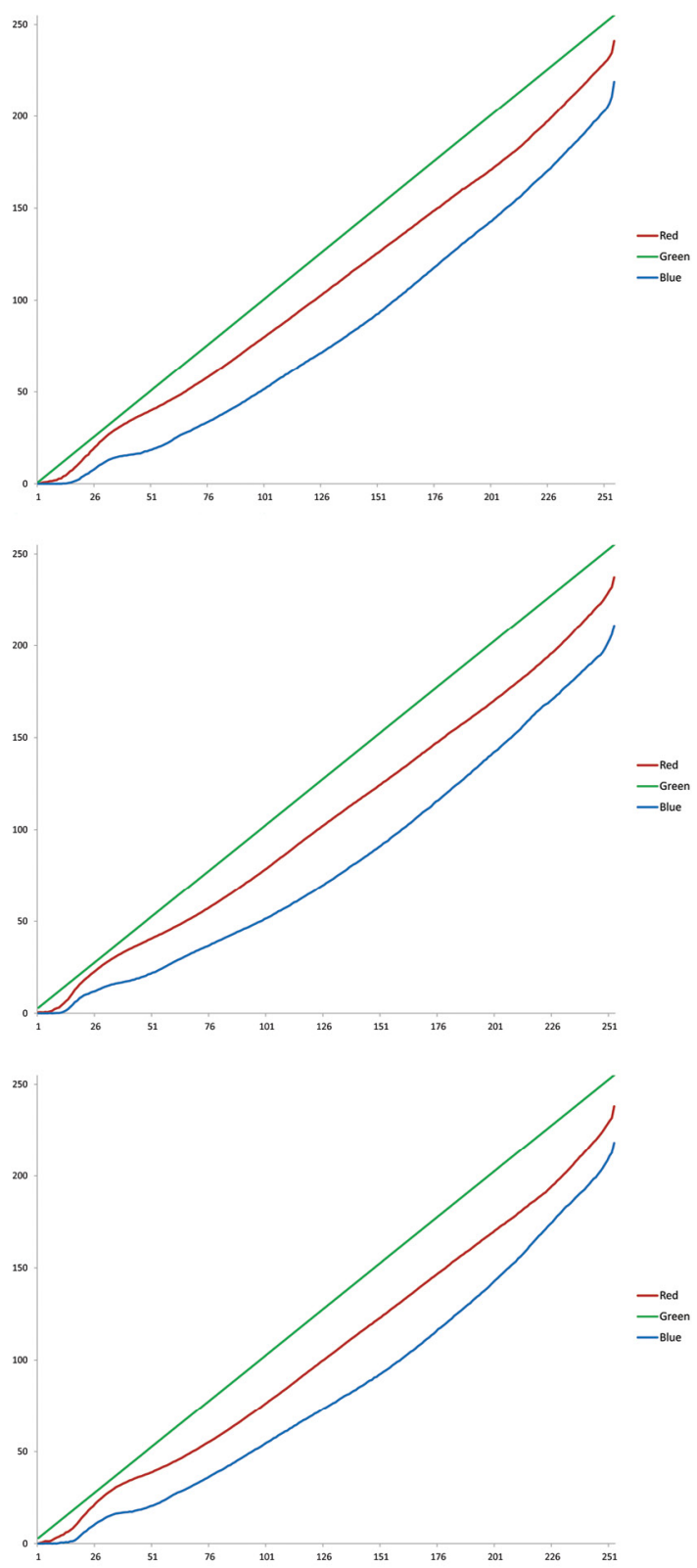

Figure S10. Effect of water stress on GSM graph in the canopies of 4 wheat cultivars. It seems that the overall result of variations in red and blue curves, lead to bottleneck and swollen forms, e.g. due to higher reflection of blue light under complete shadow. It is noteworthy, that the reflection pattern is expected to be influenced by the interaction between increased evapotranspiration (water stress) and osmotic adjustment (as the stress tolerance strategy plants adopt), which also may result to different responses among cultivars. 
1st cultivar (monoculture, treatment 1).

Physiological phase:

spike emergence, (174 DAS).

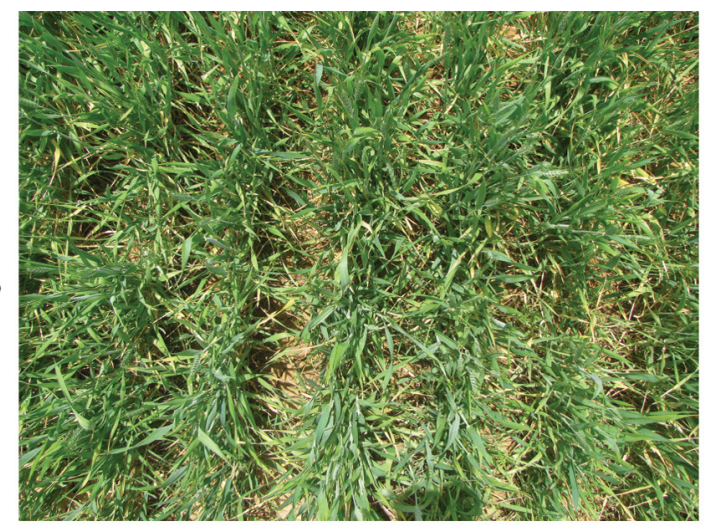

2nd cultivar (monoculture, treatment 2).

Physiological phase: spike emergence, (174 DAS).

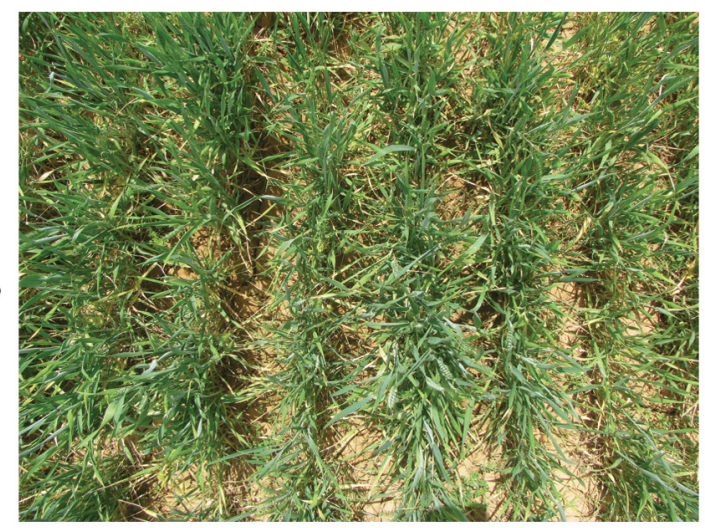

3rd cultivar (monoculture, treatment 3).

Physiological phase: late booting, (174 DAS).

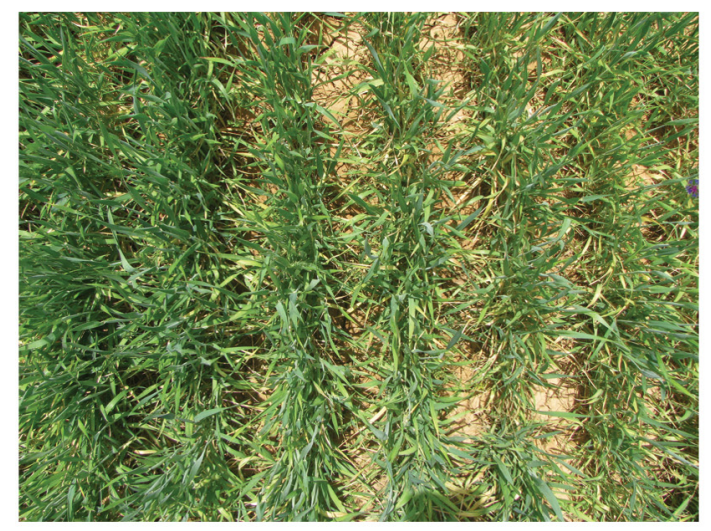

4th cultivar (monoculture, treatment 4).

Physiological phase:

booting, (174 DAS).
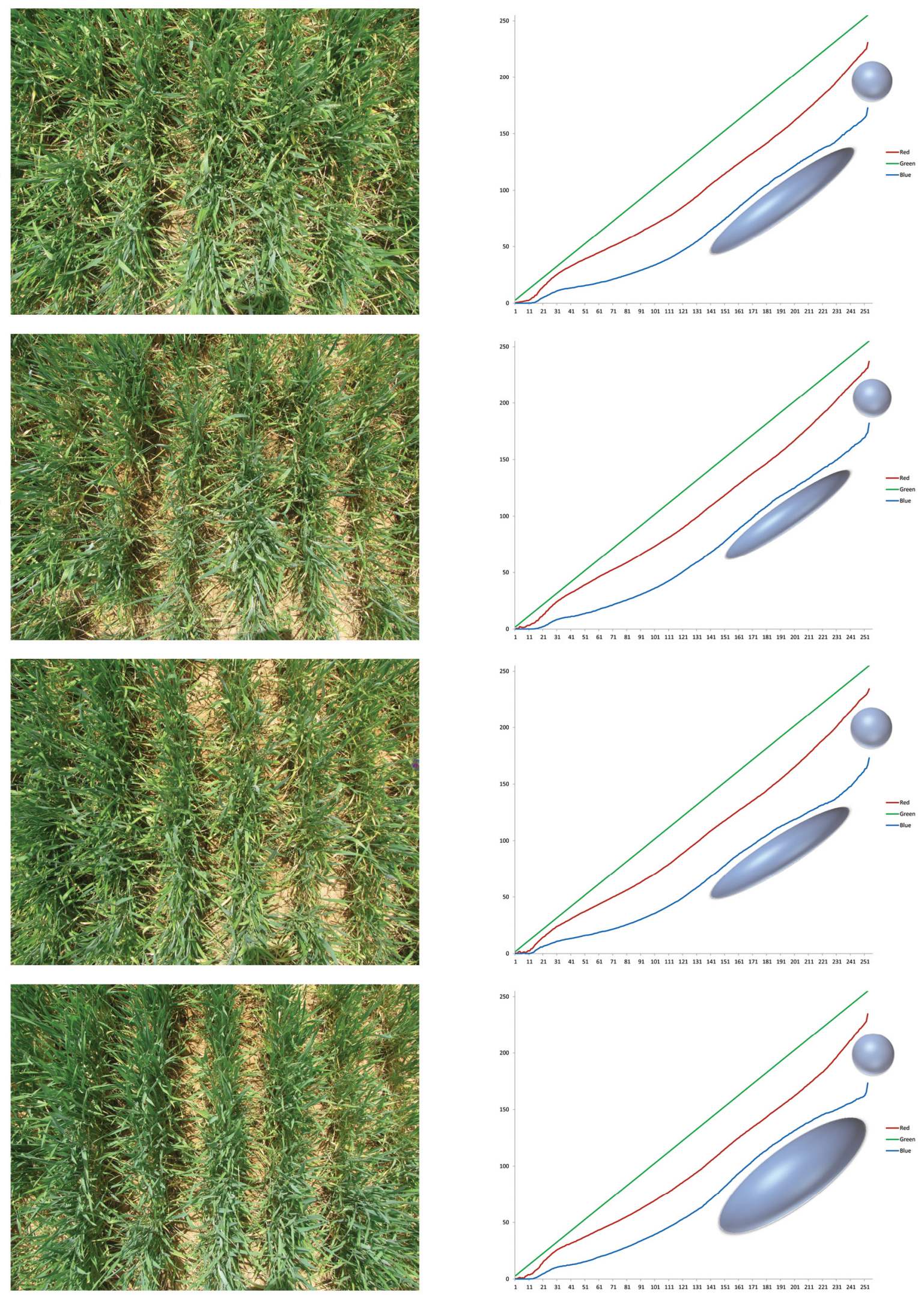

Figure S11. Images and GSM graphs of the canopies of 4 wheat cultivars, 4 days after a moderate cold stress. The most obvious impact was on the blue curve, which have resulted to a diversion from the normal trend, due to an increase in the level of mean blue values in the range of $\mathrm{B}_{24}$ curve areas (green points either exposed to direct sunlight, or positioned under partial shadow, see the manuscript). Moreover, compared to the curves of non-stress conditions, there is a considerable difference between the maximum values of red and blue (near 255 , in full sunlight). The interferences of cold stress have shown by ellipsoids and spheres of different sizes (which may be related to different tolerances of the cultivars). 
A

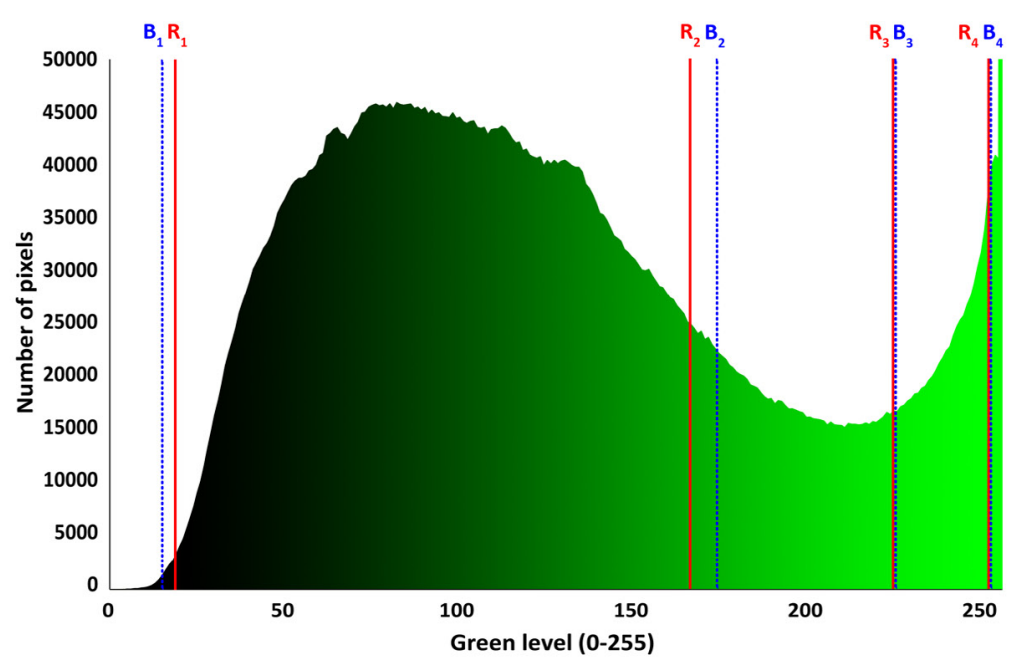

B

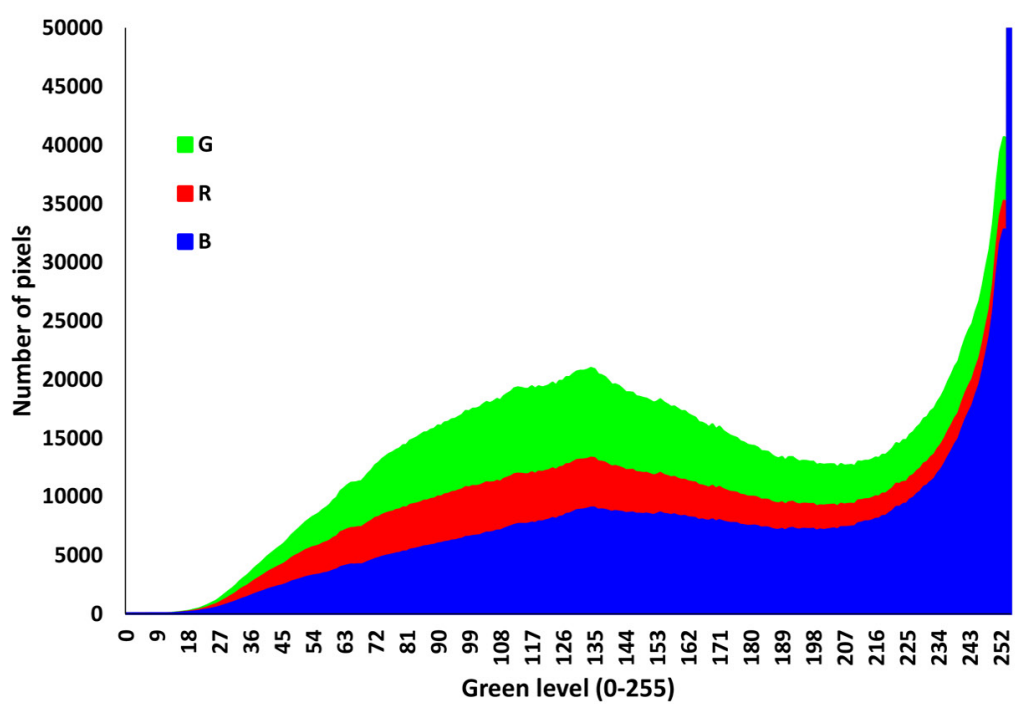

C

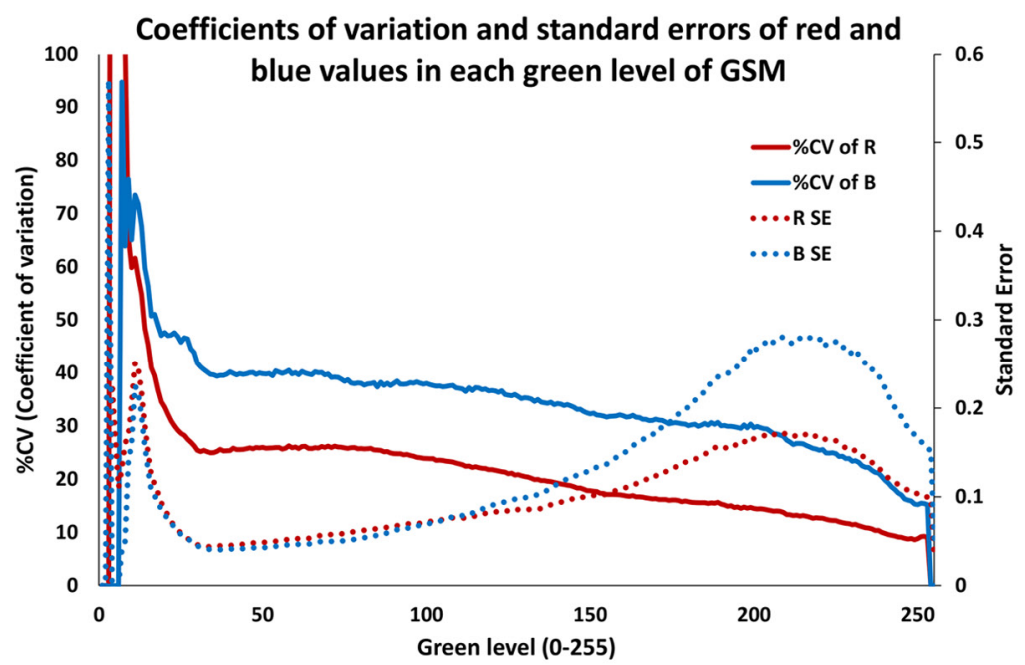

Figure S12. (A) Number of image pixels participated in drawing the GSM graph (Num. of GSM). $\mathrm{R}_{1}$ and/or $\mathrm{B}_{1}$ to $\mathrm{R}_{4}$ and/or $\mathrm{B}_{4}$ are the thresholds of segmentation type 3 . The green gradient color of the under curve area shows the GSM green level. (B) Results of "GSM-curve values $\times$ Num. of GSM'. This graph is not studied more in the present survey, however, it may provide beneficial information about canopy optics and image-derived vegetation indices (VIs). Accordingly, mean red, green, or blue values of the vegetation parts are either among the common image-derived vegetation indices themselves, or used for calculating the other ones. Such measurers are mostly calculated as a single value for the whole vegetation coverage. The present conceptual graph shows the distribution of these values across the GSM graph, i.e. represents the contribution of various kinds of sun-exposed to shaded surfaces to estimation of the described VIs. (C) Indices of dispersion calculated for GSM graph. As described in the manuscript, the GSM curves are formed based on the averaged values of red or blue colors of the vegetation part. Here, the coefficients of variation (\%) and standard errors for red and blue colors are shown. 
bioRxiv preprint doi: https://doi.org/10.1101/241786; this version posted January 13, 2019. The copyright holder for this preprint (which was not certified by peer review) is the author/funder. All rights reserved. No reuse allowed without permission.
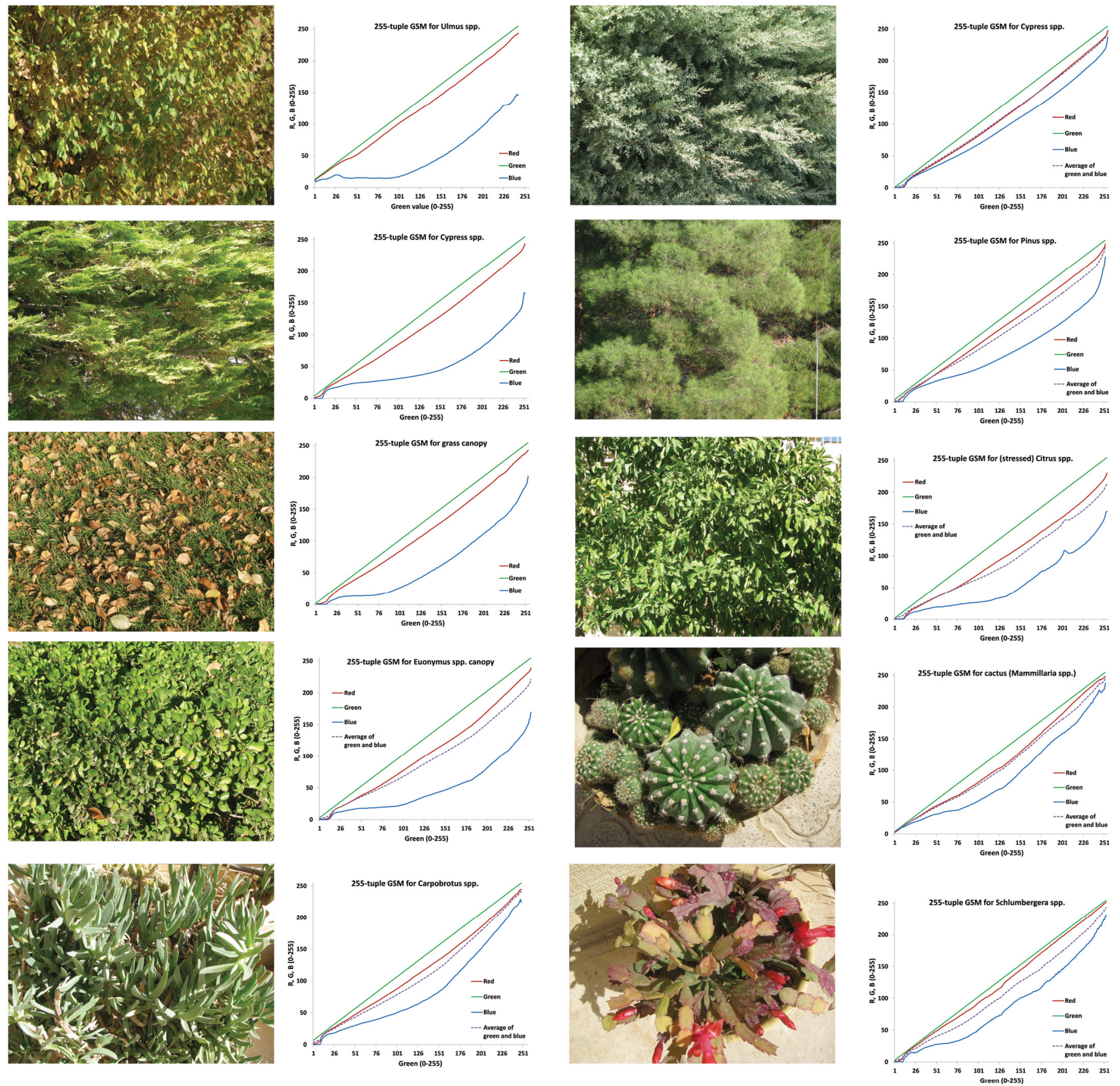

Figure S13. Examples of GSM graphs drawn for some other plant species. 
Table S1. List of GSM-curve derived attributes used in the present study, categorized based on the type of the main characteristics they are originated.

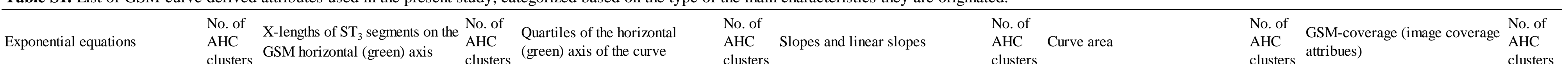

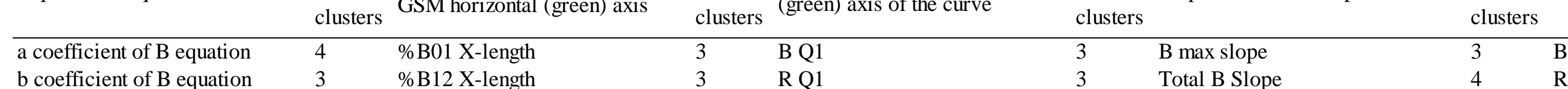
$b$ coefficient of $B$ equation
a coefficient of $R$ equation $\mathrm{BR}^{2}$
$\mathrm{~B}$ RMSE

R R
R RMSE

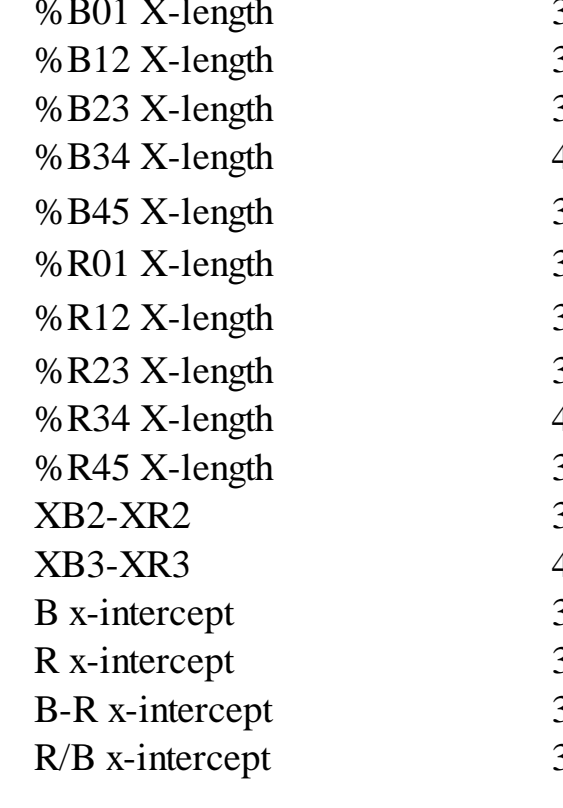

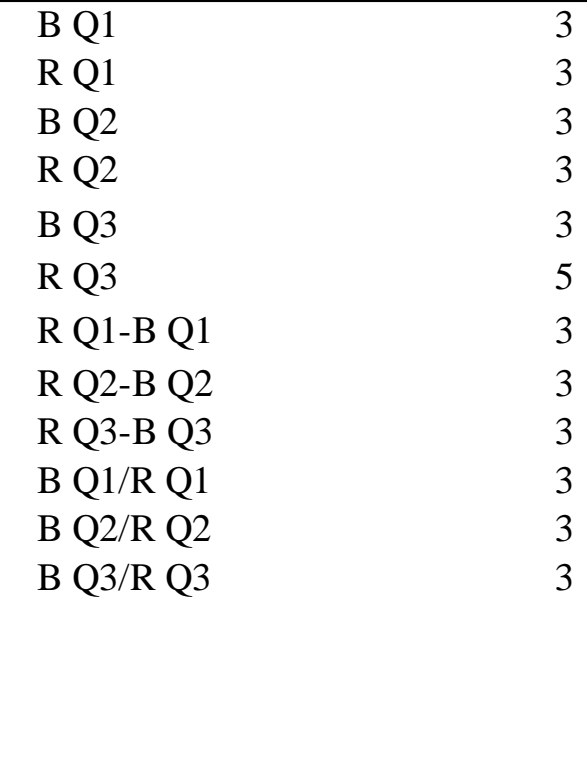

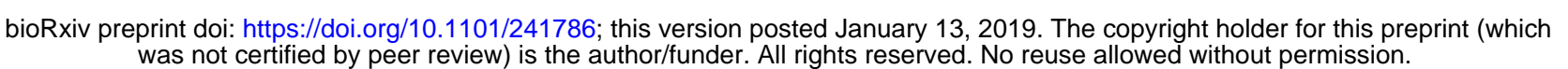

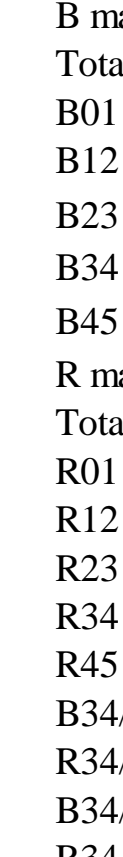

B max slope
Total B Slope
B01 slope

B01 slope
B12 slope

B23 slope

B34 slope
B45 slope

R max slope
Total R Slope

R01 slope
R1 slope

R12 slope
R23 slope
R34

R43 slope

B34/B12 Slope

R34/R14 Slope

R34/1334 Slope

R24/B24 Slope
R12/B12 Slope

Total B LSlope

B45 LSlope
B34 Lslope
B23

B12 Lslope

B01 Lslope
Total R LSlope
R45 Lslope

R45 Lslope

R23 Lslope
R12 Lslope

R01 Lslope
Slope B12*B34

Slope B12*BB34
SB12×SB34)-SB23
SB34-Invsd SB12

SB34-Invsd SB12
Slope R12*R34

(SR12×SR34)-SR23

SR34-IInvd SR12
B resSlope/R resSlope

B resSlope/R resSlope
Mean R\&B Slope

Total R-B Lslope

Total B/R Lslope
B45/R45 Lslope

B34/R34 Lslope

B23/R23 Lslope

B12/R12 Llope
B01/R01 Lslope

Total B slope/ B Lslope

B45 Slope/ B45 Lslope

B23 Slope/B23 Lslope

B12 Slope/B12 Lslope

B01 Slope/B01 Lslope

Total R Rlope/ R Llope
R45 Slope/R45 Llope

R34 Slope/R34 Lslope

R23 Slope/R23 Lslope

R01 Slope/ R01 Lssope

R23/B23 Slope

Mean of total R\&B
R45/R34 Slope

B45/B34 Slope

For more information about the GSM attributes, see the Fig. S3; No. of AHC clusters shows the number of clusters of the attributes used in the quantitativive computation approach (i.e. the 3rd approach), based on the Aggolomerative hierarchical clustering

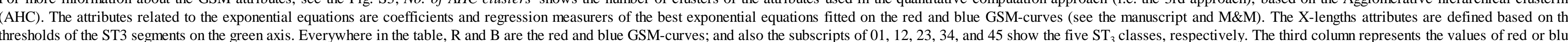
curves at the first $\left(Q_{1}\right)$ to third $\left(Q_{3}\right)$ quartiles of the horizontal axis. "Max slope" means the maximum slope through the whole curve, and "Lslope" stands for the linear slope. Moreover, in the "Slopes and linear slopes column, "s" and "invsd" are the abbreviates for "llope" and "inversed value",

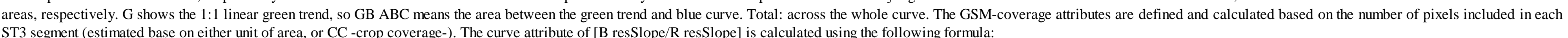

B resslope $/$ R resSlope $=\left(\right.$ Slope $B_{34}-1 /\left(\right.$ Slope $\left.\left._{12}\right)\right) /\left(\right.$ Slope $R_{34}-1 /\left(\right.$ Slope $\left.\left.R_{12}\right)\right)$ 
Table S2. Correlations of GSM curve-derived attributes with grain yield.

\begin{tabular}{|c|c|c|c|}
\hline \multicolumn{2}{|l|}{ Possitive correlations } & \multicolumn{2}{|l|}{ Negative correlations } \\
\hline GSM Attribute & Correlation $(\mathrm{R})$ & GSM Attribute & Correlation $(\mathrm{R})$ \\
\hline B23 Slope/B23 Lslope & 0.28103348 & $\bar{B} 23$ AUC & -0.302475716 \\
\hline $\mathrm{R} / \mathrm{B}$ x-intercept & 0.246039953 & $\% \mathrm{~B} 23 \mathrm{X}$-length & -0.279796196 \\
\hline $\mathrm{R} x$-intercept & 0.243504195 & GB23 ABC & -0.269219228 \\
\hline B02/B04 AUC & 0.236640828 & $\%$ Num B23/unit of area & -0.267200086 \\
\hline GR23/GB23 AUC & 0.235341203 & $\%$ Num B01/unit of area & -0.263525141 \\
\hline Num R23/Num B23 & 0.234939163 & $\%$ Num B01/CC & -0.26293073 \\
\hline B12 AUC & 0.229901832 & $\%$ Num B23/CC & -0.223217476 \\
\hline GB34 ABC & 0.222796987 & Num B23/Num B05 & -0.222656973 \\
\hline$\%$ B12 X-length & 0.218924097 & $\%$ Num R23/unit of area & -0.209878261 \\
\hline R01 slope & 0.212679891 & B23 Lslope & -0.205899512 \\
\hline R23 Slope/R23 Lslope & 0.201070383 & B23/R23 Lslope & -0.200025685 \\
\hline B01/R01 Lslope & 0.192363185 & $\%$ Num R01/unit of area & -0.169321897 \\
\hline $\mathrm{GB} 12 \mathrm{ABC}$ & 0.187997479 & B12 Slope/B12 Lslope & -0.164919 \\
\hline B01 Lslope & 0.173441862 & $\%$ B01 X-length & -0.162725317 \\
\hline R02/R04 AUC & 0.171477186 & GB01 ABC & -0.161139281 \\
\hline$\%$ R12 X-length & 0.171114123 & GR01 ABC & -0.159807019 \\
\hline$\%$ B34 X-length & 0.170652638 & B45 slope & -0.158091753 \\
\hline R01 Lslope & 0.169119181 & GR23 ABC & -0.154584265 \\
\hline$\%$ Num B12/CC & 0.15195497 & $\%$ R23 X-length & -0.154108964 \\
\hline R12 AUC & 0.150247381 & Num R34/Num B34 & -0.151911926 \\
\hline R23/B23 Slope & 0.148542214 & B23 slope & -0.141281875 \\
\hline R23-B23 AUC & 0.141270786 & $\%$ Num R01/CC & -0.141071763 \\
\hline$\%$ Num B34/CC & 0.141160592 & GR34/GB34 AUC & -0.137233569 \\
\hline Num B35/Num B05 & 0.13984841 & ( $\mathrm{R}$ to $\mathrm{B}$ ratio34)/(R to B ratio12) & -0.131736941 \\
\hline B34 AUC & 0.133884132 & R23 AUC & -0.131365989 \\
\hline GR12 ABC & 0.132633998 & R34-B34 AUC & -0.123703226 \\
\hline$\%$ Num R12/CC & 0.123418012 & $\%$ Num B45/unit of area & -0.120156926 \\
\hline R45 Slope/R45 Lslope & 0.114977523 & $\%$ Num R12/unit of area & -0.119039087 \\
\hline Total R LSlope & 0.104965286 & $\%$ Num R23/CC & -0.117046652 \\
\hline R45 Lslope & 0.095029026 & B RMSE & -0.116712642 \\
\hline B resSlope/R resSlope & 0.092704087 & Num R23/Num R05 & -0.115571958 \\
\hline (SB12×SB34)-SB23 & 0.091871246 & R01 Slope/ R01 Lslope & -0.108816256 \\
\hline XB3-XR3 & 0.0917346 & Total R slope/ R Lslope & -0.104170332 \\
\hline Total R-B Lslope & 0.091700457 & B Q2 & -0.093187376 \\
\hline B01 slope & 0.086995764 & B Q1 & -0.092947076 \\
\hline R45/R34 Slope & 0.085520358 & a coefficient of $B$ equation & -0.09132316 \\
\hline \multicolumn{4}{|c|}{ 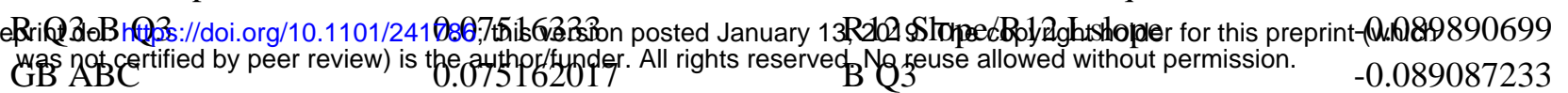 } \\
\hline R45-B45 AUC & 0.073858737 & Total B/R Lslope & -0.087588177 \\
\hline R24-B24 AUC & 0.073777702 & $\%$ Num R45/unit of area & -0.085791483 \\
\hline GR45 ABC & 0.072890304 & B45/B34 Slope & -0.084745723 \\
\hline R Q2-B Q2 & 0.071398599 & B-R x-intercept & -0.083147307 \\
\hline$\%$ Num B34/unit of area & 0.0707981 & B Q2/R Q2 & -0.083032665 \\
\hline R45 AUC & 0.069441089 & B Q1/R Q1 & -0.082719907 \\
\hline R Q1-B Q1 & 0.06775137 & B Q3/R Q3 & -0.082236841 \\
\hline$b$ coefficient of $B$ equation & 0.066982537 & Num R12/Num B12 & -0.080430261 \\
\hline $\mathrm{RB} A B C$ & 0.061355098 & R23 Lslope & -0.076532908 \\
\hline B34/R34 Lslope & 0.058453455 & B AUC & -0.075082287 \\
\hline$\%$ R45 X-length & 0.058113155 & GR12/GB12 AUC & -0.075032035 \\
\hline B01 AUC & 0.055109154 & B01 Slope/B01 Lslope & -0.072814984 \\
\hline R R2 & 0.053413205 & B45 Lslope & -0.067380145 \\
\hline Num R45/Num B45 & 0.050268978 & B34 Slope/B34 Lslope & -0.06662219 \\
\hline Num R01/Num B01 & 0.048450398 & R RMSE & -0.065578333 \\
\hline B34/B14 Slope & 0.046539402 & R34 Lslope & -0.059236956 \\
\hline R12 Lslope & 0.039014702 & R34/R12 Slope & -0.057710512 \\
\hline R12 slope & 0.036404271 & $\%$ Num R34/unit of area & -0.057676687 \\
\hline Total R Slope & 0.036165271 & $\mathrm{~b}$ coefficient of $\mathrm{R}$ equation & -0.053082927 \\
\hline a coefficient of $R$ equation & 0.033030764 & R34 slope & -0.052927058 \\
\hline B max slope & 0.032057297 & \%R01 X-length & -0.052580774 \\
\hline R34 Slope/R34 Lslope & 0.026917666 & R45 slope & -0.050597523 \\
\hline R Q1 & 0.026298412 & $\%$ Total GR/GB AUC & -0.047509319 \\
\hline B R2 & 0.02604125 & R12-B12 AUC & -0.046142037 \\
\hline B34/B12 Slope & 0.02234841 & R23 slope & -0.045602431 \\
\hline GR34 ABC & 0.021429415 & R34/B34 Slope & -0.045240691 \\
\hline B45 AUC & 0.020888368 & SR34-Invsd SR12 & -0.044277814 \\
\hline XB2-XR2 & 0.018344536 & $\%$ Num B45/CC & -0.04358279 \\
\hline SB34-Invsd SB12 & 0.015413617 & Slope R12*R34 & -0.043542905 \\
\hline R12/B12 Slope & 0.013837518 & Total B Slope & -0.040952192 \\
\hline R Q2 & 0.013446849 & $\%$ Num B12/unit of area & -0.040463746 \\
\hline B34 Lslope & 0.013108189 & R34/R14 Slope & -0.039944744 \\
\hline Mean R\&B Slope & 0.010921505 & GR01/GB01 AUC & -0.035143333 \\
\hline Mean of total R\&B Lslope & 0.010921505 & Total B LSlope & -0.033373582 \\
\hline R01 AUC & 0.010083955 & B45/R45 Lslope & -0.030799722 \\
\hline GB45 ABC & 0.00988606 & B45 Slope/B45 Lslope & -0.021545026 \\
\hline $\mathrm{R}$ max slope & 0.009758379 & Total B slope/ B Lslope & -0.021442128 \\
\hline \%Num R34/CC & 0.009301824 & B12/R12 Lslope & -0.020917444 \\
\hline \% B45 X-length & 0.008947291 & (SR12×SR34)-SR23 & -0.015116042 \\
\hline Num R35/Num R05 & 0.008784889 & R24/B24 Slope & -0.014560008 \\
\hline B34 slope & 0.008291367 & R Q3 & -0.012713589 \\
\hline GR45/GB45 AUC & 0.007749638 & $\%$ Num R45/CC & -0.005916822 \\
\hline B12 Lslope & 0.006199024 & R34 AUC & -0.005034129 \\
\hline R01-B01 AUC & 0.00491496 & B12 slope & -0.00341403 \\
\hline$\%$ R34 X-length & 0.00386586 & B X-intercept & -0.00161062 \\
\hline \multirow[t]{2}{*}{ GR ABC } & $2.25 \mathrm{E}-04$ & R AUC & $-1.62 \mathrm{E}-04$ \\
\hline & & Slope B12*B34 & $-9.53 \mathrm{E}-06$ \\
\hline
\end{tabular}


Table S3. Correlations of GSM curve-derived attributes with No. of grains $\mathrm{m}^{-2}$.

\begin{tabular}{|c|c|c|c|}
\hline \multicolumn{2}{|l|}{ Possitive correlations } & \multicolumn{2}{|l|}{ Negative correlations } \\
\hline GSM Attribute & Correlation $(\mathrm{R})$ & GSM Attribute & Correlation $(\mathrm{R})$ \\
\hline B02/B04 AUC & 0.33561069 & B23 AUC & -0.332810342 \\
\hline B12 AUC & 0.319508907 & $\%$ Num B23/unit of area & -0.324686421 \\
\hline$\%$ B12 X-length & 0.311229311 & $\%$ B23 X-length & -0.32457646 \\
\hline GB12 ABC & 0.281708209 & $\%$ Num B01/CC & -0.293565197 \\
\hline$\%$ R12 X-length & 0.26453659 & $\%$ Num B01/unit of area & -0.293422455 \\
\hline B23 Slope/B23 Lslope & 0.261935808 & GB23 ABC & -0.289714994 \\
\hline R02/R04 AUC & 0.257272696 & $\%$ Num B23/CC & -0.286512876 \\
\hline R23 Slope/R23 Lslope & 0.250869574 & Num B23/Num B05 & -0.28642377 \\
\hline GR12 ABC & 0.250134524 & $\%$ Num R23/unit of area & -0.268449601 \\
\hline $\mathrm{R} / \mathrm{B}$ x-intercept & 0.249710178 & B12 Slope/B12 Lslope & -0.262819812 \\
\hline R x-intercept & 0.246930178 & $\%$ R23 X-length & -0.216671221 \\
\hline$\%$ Num B12/CC & 0.231941552 & $\%$ Num R01/unit of area & -0.202603942 \\
\hline R12 AUC & 0.218663936 & R23 AUC & -0.188878813 \\
\hline B01/R01 Lslope & 0.210712011 & B23 Lslope & -0.184356224 \\
\hline GR23/GB23 AUC & 0.207399651 & $\%$ Num R23/CC & -0.183882315 \\
\hline Num R23/Num B23 & 0.206207284 & Num R23/Num R05 & -0.183369401 \\
\hline \%Num R12/CC & 0.195078449 & B45 slope & -0.179693326 \\
\hline (SB12×SB34)-SB23 & 0.189437624 & $\%$ Num R01/CC & -0.178796215 \\
\hline GB34 ABC & 0.165982563 & B Q3 & -0.176286204 \\
\hline B01 Lslope & 0.16593308 & GR23 ABC & -0.174368474 \\
\hline $\mathrm{GB} \mathrm{ABC}$ & 0.157955287 & B23/R23 Lslope & -0.166659546 \\
\hline R01 slope & 0.157541363 & B Q2 & -0.16625478 \\
\hline B34/B14 Slope & 0.140398351 & B AUC & -0.157579369 \\
\hline R45 Slope/R45 Lslope & 0.137604507 & B Q1 & -0.153544355 \\
\hline B34/R34 Lslope & 0.130048098 & GR01 ABC & -0.153062878 \\
\hline R01 Lslope & 0.125931295 & a coefficient of $B$ equation & -0.142057367 \\
\hline R23/B23 Slope & 0.123070709 & GB01 ABC & -0.140073394 \\
\hline B34 Lslope & 0.117148334 & Total R slope/ R Lslope & -0.138090787 \\
\hline SB34-Invsd SB12 & 0.11659249 & R Q3 & -0.128289994 \\
\hline R23-B23 AUC & 0.116439388 & Num R12/Num B12 & -0.127681685 \\
\hline B34 slope & 0.112392465 & Total R Slope & -0.127157136 \\
\hline B34/B12 Slope & 0.109944734 & $\%$ B01 X-length & -0.125178871 \\
\hline B01 AUC & 0.109069863 & B23 slope & -0.121888036 \\
\hline Slope B12*B34 & 0.104691915 & R12 Slope/R12 Lslope & -0.119789126 \\
\hline Num B35/Num B05 & 0.101643624 & Total B Slope & -0.117356818 \\
\hline R45 Lslope & 0.101498027 & R34/B34 Slope & -0.117043532 \\
\hline GR ABC & 0.100715225 & B Q3/R Q3 & -0.114011319 \\
\hline \multicolumn{4}{|c|}{ 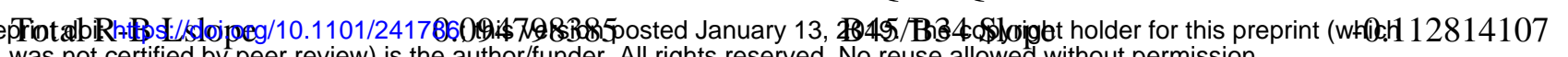 } \\
\hline $\begin{array}{l}\text { was not certified by peer review) is } \\
\mathrm{B} \text { max slope }\end{array}$ & 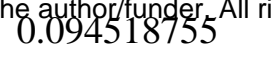 & Bo Q21 R Q2 2 & -0.106741987 \\
\hline R24-B24 AUC & 0.093746144 & B Q1/R Q1 & -0.1020848 \\
\hline \%B34 X-length & 0.089286532 & R01 Slope/ R01 Lslope & -0.100905465 \\
\hline R Q3-B Q3 & 0.085722978 & R AUC & -0.100332949 \\
\hline B01 slope & 0.081011679 & Total B LSlope & -0.099439722 \\
\hline $\mathrm{R}$ max slope & 0.076769157 & B RMSE & -0.099001046 \\
\hline$\%$ Num B34/CC & 0.0708715 & Total B/R Lslope & -0.096844828 \\
\hline R Q2-B Q2 & 0.070606103 & R23 Lslope & -0.094913944 \\
\hline b coefficient of B equation & 0.070372883 & R RMSE & -0.094877863 \\
\hline R34 Slope/R34 Lslope & 0.067061508 & R24/B24 Slope & -0.092610117 \\
\hline RB ABC & 0.066558777 & Mean R\&B Slope & -0.090371511 \\
\hline$\%$ Num R45/CC & 0.063137633 & Mean of total R\&B Lslope & -0.090371511 \\
\hline R45/R34 Slope & 0.062451153 & Num R34/Num B34 & -0.086778236 \\
\hline (SR12×SR34)-SR23 & 0.062206694 & GR12/GB12 AUC & -0.08279118 \\
\hline R Q1-B Q1 & 0.06162523 & B01 Slope/B01 Lslope & -0.078782281 \\
\hline R34/R14 Slope & 0.050178872 & B-R x-intercept & -0.075774545 \\
\hline GR34 ABC & 0.049087075 & GR34/GB34 AUC & -0.07564663 \\
\hline R R2 & 0.047399676 & B45 Slope/B45 Lslope & -0.070995035 \\
\hline XB3-XR3 & 0.046184534 & R34-B34 AUC & -0.068131438 \\
\hline$\%$ Num B45/CC & 0.046098536 & R Q2 & -0.066962152 \\
\hline B34 AUC & 0.044384218 & ( $\mathrm{R}$ to $\mathrm{B}$ ratio34)/(R to $\mathrm{B}$ ratio12) & -0.066502949 \\
\hline$\%$ Num B12/unit of area & 0.042499247 & $\%$ Num R34/unit of area & -0.062887912 \\
\hline B12 Lslope & 0.04163348 & $\mathrm{~b}$ coefficient of $\mathrm{R}$ equation & -0.058626519 \\
\hline GR45 ABC & 0.040511812 & R23 slope & -0.056322447 \\
\hline R45-B45 AUC & 0.038829329 & Total B slope/ B Lslope & -0.056133546 \\
\hline Num R01/Num B01 & 0.038066485 & R12-B12 AUC & -0.054144518 \\
\hline B12/R12 Lslope & 0.037505949 & $\%$ Num R12/unit of area & -0.051622521 \\
\hline R34 slope & 0.030219024 & $\%$ R01 X-length & -0.050386954 \\
\hline R34/R12 Slope & 0.027464275 & B45 Lslope & -0.046277869 \\
\hline B12 slope & 0.027390291 & B R2 & -0.0424805 \\
\hline Num R35/Num R05 & 0.025775907 & R45 slope & -0.042270947 \\
\hline Num R45/Num B45 & 0.025616134 & GR01/GB01 AUC & -0.039759852 \\
\hline R34 Lslope & 0.024473912 & Total R LSlope & -0.035516679 \\
\hline SR34-Invsd SR12 & 0.023865923 & R34 AUC & -0.034176448 \\
\hline Slope R12*R34 & 0.02316782 & R12/B12 Slope & -0.032947064 \\
\hline R01 AUC & 0.018590856 & R Q1 & -0.030985161 \\
\hline B resSlope/R resSlope & 0.017713905 & $\%$ Num B45/unit of area & -0.021992694 \\
\hline R45 AUC & 0.015321361 & $\%$ B45 X-length & -0.018914623 \\
\hline$\%$ Num B34/unit of area & 0.011682495 & R12 slope & -0.01497436 \\
\hline B34 Slope/B34 Lslope & 0.009919946 & GR45/GB45 AUC & -0.014506435 \\
\hline R01-B01 AUC & 0.008358384 & $\%$ R34 X-length & -0.014313915 \\
\hline B x-intercept & 0.007652113 & \%Total GR/GB AUC & -0.012237053 \\
\hline$\%$ R45 X-length & 0.004493168 & R12 Lslope & -0.010550307 \\
\hline B45/R45 Lslope & 0.001678527 & XB2-XR2 & -0.010223914 \\
\hline & & a coefficient of $R$ equation & -0.009921942 \\
\hline & & $\%$ Num R34/CC & -0.00968426 \\
\hline & & B45 AUC & -0.009486654 \\
\hline & & $\%$ Num R45/unit of area & -0.005870561 \\
\hline & & GB45 ABC & -0.002347801 \\
\hline
\end{tabular}


Table S5. List of important pairwise correlations between GSM curve-derived attributes.

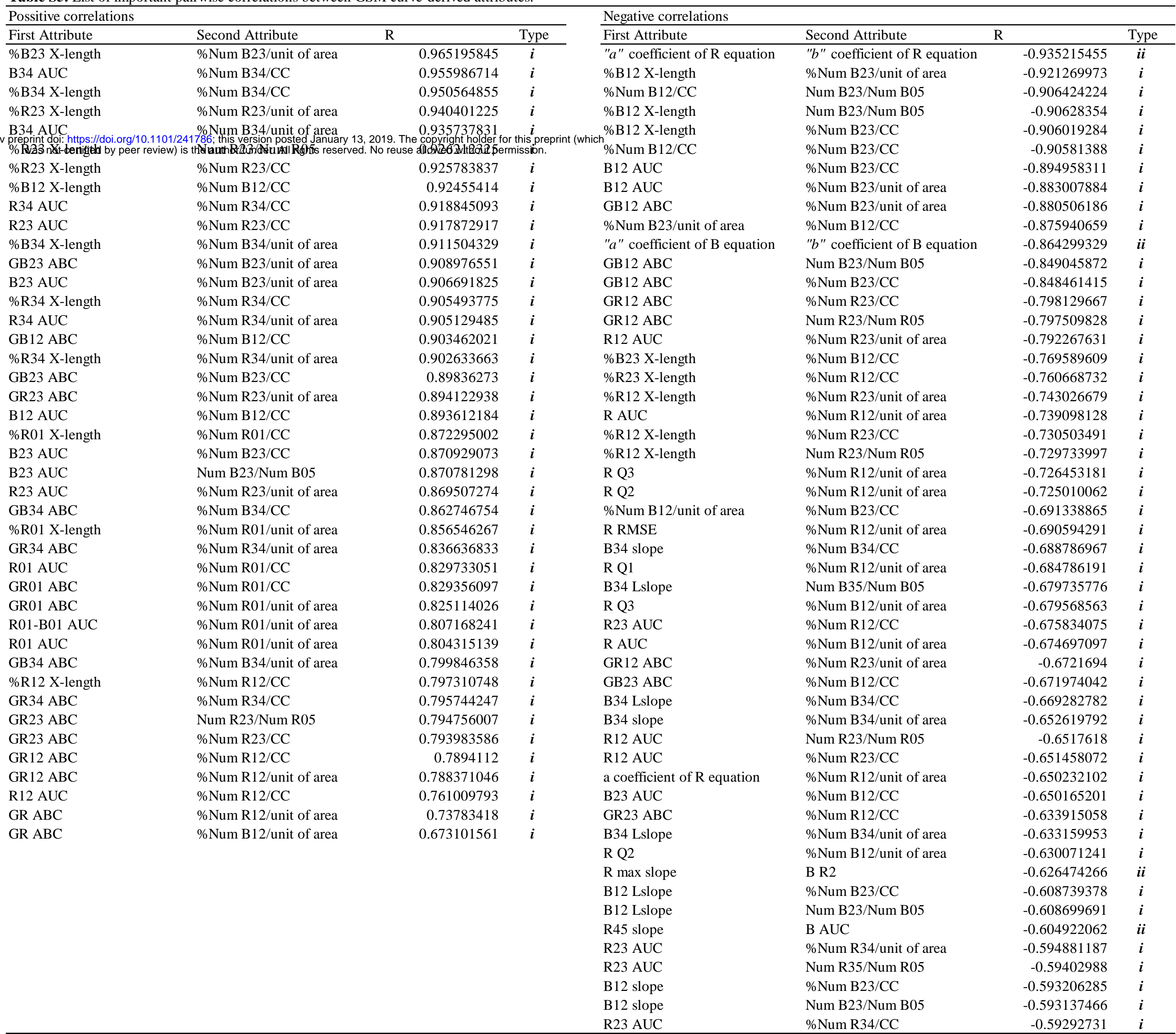

The important pairwise correlations among the total number of 169 GSM-curve characteristics. For providing this table, the strongest 400 positive and 400 negative correlations were evaluated, and the obvious relationships were filter out (for example, the high correlation between B $\mathrm{Q}_{2}$ and B AUC). The Type column shows $i$ : the relationship between the GSM-coverage attributes and other curve characteristics. $i$ : the relationships between other types of attributes. 
bioRxiv preprint doi: https://doi.org/10.1101/241786; this version posted January 13, 2019. The copyright holder for this preprint (which was not certified by peer review) is the author/funder. All rights reserved. No reuse allowed without permission.

Table S6. Model performances in predicting the correct classes of grain yields using GSM-coverage attributes as inputs in the $3^{\text {rd }}$ prediction approach.

\begin{tabular}{|c|c|c|c|c|c|c|}
\hline \multirow[b]{2}{*}{ Model } & \multicolumn{6}{|c|}{ Model performance } \\
\hline & & Obs. class 3 & Obs. class 4 & Obs. class 1 & Obs. class 2 & $\begin{array}{l}\text { Prediction } \\
\text { accuracy }(\%)\end{array}$ \\
\hline \multirow[t]{4}{*}{ Deep Learning } & Pred. class 3 & 5 & 1 & 2 & 1 & \multirow{4}{*}{87} \\
\hline & Pred. class 4 & 0 & 3 & 0 & 0 & \\
\hline & Pred. class 1 & 0 & 0 & 20 & 0 & \\
\hline & Pred. class 2 & 0 & 0 & 2 & 11 & \\
\hline \multirow[t]{4}{*}{ General Linear Model } & Pred. class 3 & 3 & 0 & 0 & 0 & \multirow{4}{*}{93} \\
\hline & Pred. class 4 & 0 & 4 & 0 & 0 & \\
\hline & Pred. class 1 & 2 & 0 & 24 & 1 & \\
\hline & Pred. class 2 & 0 & 0 & 0 & 11 & \\
\hline \multirow[t]{4}{*}{ ID3 } & Pred. class 3 & 5 & 0 & 0 & 0 & \multirow{4}{*}{100} \\
\hline & Pred. class 4 & 0 & 4 & 0 & 0 & \\
\hline & Pred. class 1 & 0 & 0 & 24 & 0 & \\
\hline & Pred. class 2 & 0 & 0 & 0 & 12 & \\
\hline \multirow[t]{4}{*}{ Decision Tree } & Pred. class 3 & 5 & 1 & 1 & 1 & \multirow{4}{*}{82} \\
\hline & Pred. class 4 & 0 & 0 & 0 & 0 & \\
\hline & Pred. class 1 & 0 & 3 & 23 & 2 & \\
\hline & Pred. class 2 & 0 & 0 & 0 & 9 & \\
\hline \multirow[t]{4}{*}{ Random Forest } & Pred. class 3 & 5 & 0 & 0 & 0 & \multirow{4}{*}{100} \\
\hline & Pred. class 4 & 0 & 4 & 0 & 0 & \\
\hline & Pred. class 1 & 0 & 0 & 24 & 0 & \\
\hline & Pred. class 2 & 0 & 0 & 0 & 12 & \\
\hline \multirow[t]{4}{*}{ Gradient Boosted Trees } & Pred. class 3 & 4 & 0 & 0 & 0 & \multirow{4}{*}{96} \\
\hline & Pred. class 4 & 0 & 4 & 0 & 0 & \\
\hline & Pred. class 1 & 1 & 0 & 24 & 1 & \\
\hline & Pred. class 2 & 0 & 0 & 0 & 11 & \\
\hline Overall & & & & & & 93 \\
\hline
\end{tabular}

There were 4,5, 12, and 24 samples for the qualitative classes 4,3,2, and 1 in the sub-dataset used for model validation; which are (in the order of class 4 to 1) the highest to lowest yielding groups, respectively (based on AHC, Agglomerative Hierarchical Clustering of values sorted in ascending order). Obs. and Pred. stand for the observed and predicted classes, respectively. 
Table S7. Model performances in predicting the correct classes of No. of grains $\mathrm{m}^{-2}$ using GSM-coverage attributes as inputs in the $3^{\text {rd }}$ prediction approach.

\begin{tabular}{|c|c|c|c|c|c|}
\hline \multirow[b]{2}{*}{ Model } & \multicolumn{5}{|c|}{ Model performance } \\
\hline & & Obs. class 2 & Obs. class 3 & Obs. class 1 & $\begin{array}{l}\text { Prediction } \\
\text { accuracy }(\%)\end{array}$ \\
\hline \multirow[t]{3}{*}{ Deep Learning } & Pred. class 2 & 13 & 0 & 2 & \\
\hline & Pred. class 3 & 0 & 0 & 0 & 96 \\
\hline & Pred. class 1 & 0 & 0 & 30 & \\
\hline \multirow[t]{3}{*}{ General Linear Model } & Pred. class 2 & 13 & 0 & 0 & \\
\hline & Pred. class 3 & 0 & 0 & 0 & 100 \\
\hline & Pred. class 1 & 0 & 0 & 32 & \\
\hline \multirow[t]{3}{*}{ ID3 } & Pred. class 2 & 13 & 0 & 0 & \\
\hline & Pred. class 3 & 0 & 0 & 0 & 100 \\
\hline & Pred class 1 & 0 & 0 & 32 & \\
\hline \multirow[t]{3}{*}{ Decision Tree } & Pred. class 2 & 8 & 0 & 2 & \\
\hline & Pred. class 3 & () & 0 & 0 & 84 \\
\hline & Pred. class 1 & 5 & 0 & 30 & \\
\hline \multirow[t]{3}{*}{ Random Forest } & Pred. class 2 & 13 & 0 & 0 & \\
\hline & Pred. class 3 & 0 & 0 & 0 & 100 \\
\hline & Pred. class 1 & 0 & 0 & 32 & \\
\hline \multirow[t]{3}{*}{ Gradient Boosted Trees } & Pred. class 2 & 9 & 0 & 0 & \\
\hline & Pred. class 3 & 0 & 0 & 0 & 91 \\
\hline & Pred. class 1 & 4 & 0 & 32 & \\
\hline Overall & & & & & 95 \\
\hline
\end{tabular}

There were 13,0, and 32 samples for the qualitative classes $4,3,2$, and 1 in the sub-dataset used for model validation; which are (in the order of class 4 to 1) the highest to lowest yielding groups, respectively (based on AHC, Agglomerative Hierarchical Clustering of values sorted in ascending order). It is notable that the randomly division of the 90 plots into two 45-sample sets for model training and validation purposes, caused that the training set had two samples of class 3 , while the validation set lacked this class. Obs. and Pred. stand for the observed and predicted classes, respectively. 